\title{
Results of the Mount Stromlo Abell cluster supernova search ${ }^{\star \star \star}$
}

\author{
L. M. Germany ${ }^{1,2}$, D. J. Reiss ${ }^{3}$, B. P. Schmidt ${ }^{4}$, C. W. Stubbs ${ }^{3}$, and N. B. Suntzeff ${ }^{5}$ \\ ${ }^{1}$ European Southern Observatory, Casilla 19001, Santiago, Chile \\ 2 Visiting Astronomer, Cerro Tololo Inter-American Observatory, National Optical Astronomy Observatories. The National \\ Optical Astronomy Observatories is operated by the Associated Universities for Research in Astronomy (AURA), Inc. under \\ cooperative agreement with the National Science Foundation \\ 3 University of Washington, Box 351580, Seattle, WA, USA \\ ${ }^{4}$ Research School of Astronomy and Astrophysics, Australian National University, Canberra, Australia \\ 5 Cerro Tololo Inter-American Observatory, National Optical Astronomy Observatories, Casilla 603, La Serena, Chile
}

Received 9 September 2002 / Accepted 13 October 2003

\begin{abstract}
We report the results of the Mount Stromlo Abell cluster supernova search, a three-year project to find supernovae ( $\mathrm{SNe}$ ) in a well-defined sample of high-density, southern Abell clusters with redshifts $0.02 \leq z \leq 0.08$. The search discovered $48 \mathrm{SNe}, 23$ of which were consistent with SNe of type Ia (SNIa). Of these, 15 were spectroscopically classified SNIa, with the remaining $8 \mathrm{SNe}$ tentatively classified as SNIa via the goodness of fit of the template light curves. This paper describes the methods employed to produce the light curves for the SNe discovered during the Mount Stromlo Abell cluster supernova search. We derive the redshift-independent distances to the SNIa from the search, as well as a large sample of other wellobserved SNIa taken from the literature via a modified $\Delta m_{15}$ template light curve fitting technique. This technique and the problems encountered in using it are described in detail. We construct a Hubble diagram for SNIa and show that our data with $z<0.2$ are consistent with an accelerating universe. Using current Cepheid HST calibrations, we estimate that the value of the Hubble constant lies between $53<H_{0}<83 \mathrm{~km}^{-1} \mathrm{~s}^{-1} \mathrm{Mpc}^{-1}$, with the large spread almost entirely due to possible systematic errors in the Cepheid calibration.
\end{abstract}

Key words. cosmology: observations - supernovae: general - surveys

\section{Introduction}

Over the past decade, research into SNIa has shown these objects to be one of the best extra-galactic distance indicators available, with a precision of $5-7 \%$ of the distance to each object (Hamuy et al. 1996a; Perlmutter et al. 1997; Riess et al. 1995, 1996). Although it has been well established that SNIa are not perfect standard candles (e.g. Phillips 1993), by accounting for the correlation between the intrinsic brightness and the rate at which the light curve decays, their precision out to large distances is unparalleled. The principal difficulty in their use as distance indicators lies in their transient nature, appearing unpredictably at a random position in the sky. However, ever-increasing numbers of SN searches are uncovering more

Send offprint requests to: L. M. Germany,

e-mail: lgermany@eso.org

* The local standard star photometry in $B V(R I)_{\mathrm{KC}}$ and the photometry in both fluxes and magnitudes for each $\mathrm{SN}$ are only available in electronic form at the CDS via anonymous $\mathrm{ftp}$ to cdsarc.u-strasbg.fr $(130.79 .127 .5)$ or via

http://cdsweb.u-strasbg.fr/cgi-bin/qcat?J/A+A/415/863

$\star \star$ Appendices $\mathrm{A}$ and $\mathrm{B}$ are only available in electronic form at http://www. edpsciences.org and more young SNe suitable for distance work, with $299 \mathrm{SNe}$ discovered in 2002 alone.

The Mount Stromlo Abell cluster supernova search (Reiss et al. 1998; hereafter Paper I) ran for 3.5 years (from 1996-1999) and was designed to search for intermediateredshift SNe. Earlier photographic surveys that explored this redshift range (Maza et al. 1981; Hamuy et al. 1993b) led to the fundamental calibrations of the intrinsic luminosities of SNIa (Phillips 1993; Hamuy et al. 1996c), however, the discovery statistics of the supernovae were clearly biased against finding SNe near the centres of host galaxies (Wang et al. 1997; Hamuy \& Pinto 1999) by the Shaw effect (Shaw 1979). Modern detectors and image subtraction techniques have largely overcome the problem of discovery bias, but most of the systematic SN searches now conducted aim to discover either high- (e.g. Schmidt et al. 1998; Perlmutter et al. 1997) or low-redshift (e.g. Treffers et al. 1997; Schwartz 1997) SNe. This still leaves the redshift range between $0.02<z<0.2$ with an obvious bias against $\mathrm{SNe}$ that explode near high surface brightness regions. Yet it is precisely this redshift range that needs to be explored in detail in order to link the high-redshift results to low-redshift research 
Having well-understood selection criteria and search techniques which are not biased by where the $\mathrm{SNe}$ appear in the host galaxy, the Mount Stromlo Abell cluster supernova search also provides a means for the accurate measurement of $\mathrm{SN}$ rates. Since the selection effects and control times of our search are known, we can compare the rates of $\mathrm{SNe}$ of different types, both in the targeted clusters and in the field surrounding them (Reiss et al. 2003a,b).

Only one other non-photographic survey thus far has specifically searched for $\mathrm{SNe}$ in a similar redshift range. The Wise Observatory optical transients search (Gal-Yam et al. 2003) undertook a survey of rich Abell clusters in the northern hemisphere, with the primary goal of measuring $\mathrm{SN}$ rates as a function of various parameters. Concentrating on northern clusters, this search was complimentary to our own, but, with a month passing between successive epochs, was less sensitive to to discovering young $\mathrm{SNe}$. This is not a problem for $\mathrm{SN}$ rate calculations, but would certainly hinder using the SNe discovered for distance work, one of our primary objectives.

This paper presents the $\mathrm{SNe}$ discovered by the Mount Stromlo Abell cluster supernova search in Sect. 2. Section 3 describes the reduction of the followup data obtained for all the $\mathrm{SNe}$ and Sect. 4 details the processes to determine the distances to the SNIa. Section 5 focuses on the results we have obtained through the data presented here. In particular it discusses our measurement of the Hubble constant and shows that our data provide supportive evidence for an accelerating universe.

\section{Supernovae discovered}

A comprehensive description of the Mount Stromlo Abell cluster supernova search was presented in Paper I, where we detailed the instrumentation used in the search, target selection, the scheduling of search observations, and finding and identifying SN candidates.

Briefly, the Mount Stromlo and Siding Springs Observatories (MSSSO) $1.3 \mathrm{~m}$ telescope was used to target all southern, high-density clusters lying within $z \lesssim 0.08$, from the Abell et al. (1989) survey of nearby, rich galaxy clusters. Search observations were scheduled so that each cluster was revisited approximately every 10 days allowing us to find young SNIa suitable for distance work. Potential $\mathrm{SNe}$ were discovered by a search routine which processed the data automatically, with the final cuts on real SN candidates made by eye. Each potential SN candidate was reobserved and confirmed before followup observations were undertaken.

The Mount Stromlo Abell cluster SN search uncovered 48 SNe during its 3.5 year campaign (Table 1), 23 of which were found to be consistent with SNIa. Due to scheduling constraints and the faintness of the SNe (typical SNIa magnitude at maximum was $m_{V}=19$ ), only 15 of these SNIa were spectroscopically classified (see Sect. 3.1). These, and other SNe for which we obtained a spectral classification, are indicated in Table 1 with $\mathrm{a}^{\dagger}$. The remaining SNIa were classified by the goodness of fit of the template light curves (see Sect. 4.4.2) This is not a foolproof method, and although the light curves of these SNe are consistent with those of SNIa, the possibility exists that they have been misclassified as such (see Sect. 4.4.3).
Those SNe that were deemed not to be SNIa via the template fits, were not investigated further and have been assigned a typing of nIa (not Ia) in Table 1.

Based on the fits of the template light curves, it appears that most of the SNIa were discovered around maximum light. This is also confirmed for those SNIa which were spectroscopically classified, by cross-correlating the age of the SN determined from the spectra and the date of discovery of the SN. That most of our SNIa were discovered around maximum light (15 of them before maximum), is not surprising. As outlined in Paper I, we designed our search to have at most 2 weeks between successive observations of any particular field. This equates well with the rise time of a SNIa.

Table 1 also indicates with an * those SNe that appeared in a galaxy associated with the targeted Abell cluster. Cluster membership is defined by Reiss et al. $(2003 a, b)$ to be restricted to galaxies with

$\left|c z_{\mathrm{SN}}-c z_{\text {cluster }}\right|<2 \bar{\sigma}_{v}$

where $\bar{\sigma}_{v}=750 \mathrm{~km} \mathrm{~s}^{-1}$ is used for the typical velocity dispersion of these rich clusters.

Immediately obvious is that almost half of the discovered $\mathrm{SNe}$ were located behind the cluster under investigation. Again this is not surprising and can be traced back to our observing strategy. To minimise selection effects and determine accurate control times for our search, our sample is volume limited to more than a magnitude below the average brightness for a SNIa in the most distant clusters in our sample. Therefore it is not unexpected that we discover SNe behind the nearer clusters.

The other glaring omissions in Table 1 are the redshifts for SN 1998bt and SN 1998dy. These SNe had no visible host galaxy to the detection limits of the search $(V=21 \mathrm{mag})$, and the SNe themselves had faded before we could obtain a spectrum. SN $1998 \mathrm{dz}$ was also an apparently hostless SN, but we were able to obtain a spectrum of the $\mathrm{SN}$ in order to estimate the redshift of this object. Subsequent deep imaging of the field around SN 1998bt has revealed a faint galaxy at the position of the SN with $R=23.4 \pm 0.1$ (Germany \& Strolger 2003).

By far the most unusual SN discovered by the search was $\mathrm{SN}$ 1997cy. This was among the brightest $\mathrm{SNe}$ ever discovered and may have been associated with a Gamma Ray Burst (Germany et al. 2000), further contributing to the discussion of a possible connection between SNe and Gamma Ray Bursts.

\section{Observations}

Followup spectroscopic and photometric observations are crucial to the success of any SN campaign. Since SN types are based on maximum light spectra, spectroscopy is required to unequivocally determine the $\mathrm{SN}$ type and allows for the easy determination of the redshift. Photometry, obviously, is needed to trace the light curve of the SN as it fades. Both types of observation are most important immediately after the discovery of the SN. 
Table 1. List of SNe discovered by the Mount Stromlo Abell cluster SN search . * indicate those SNe which occurred within the targeted cluster. ${ }^{\dagger}$ indicates those SNe which were spectroscopically classified. The SNIa? (i.e. those without the ${ }^{\dagger}$ ) were classified through the goodness of fit of the template light curves (Sect. 4.4.2) and hence could be SNe of another type that have been misclassified. Those SNe designated nIa were deemed not to be SNIa through the template fits and were not investigated further.

\begin{tabular}{|c|c|c|c|c|c|c|c|c|}
\hline SN & Abell & Type & $z$ & Cluster $z$ & $\begin{array}{cc}\alpha & (\mathrm{J} 20\end{array}$ & 0) & Offset from & Discovery \\
\hline SN 1996af & A3879 & nIa & 0.100 & 0.067 & $22^{\mathrm{h}} 28^{\mathrm{m}} 31^{\mathrm{s}} .13$ & $-68 \circ 58^{\prime} 33^{\prime \prime} 0$ & $0.7 \mathrm{~W} 0.3 \mathrm{~S}$ & $12 / 06 / 96$ \\
\hline SN 1996ag & A3809 & Ia? & 0.140 & 0.062 & 214650.47 & -434150.4 & $1.7 \mathrm{E} 0.4 \mathrm{~S}$ & $12 / 06 / 96$ \\
\hline SN 1996aj & A3559 & $\mathrm{Ia}^{\dagger}$ & 0.110 & 0.046 & 132906.82 & -291402.0 & $4.5 \mathrm{~W} 0.4 \mathrm{~N}$ & $15 / 06 / 96$ \\
\hline SN 1996am & A3809 & $\mathrm{Ia}^{\dagger}$ & $0.065^{*}$ & 0.062 & 214607.56 & -440010.9 & $0.4 \mathrm{E} 1.3 \mathrm{~N}$ & $12 / 07 / 96$ \\
\hline SN 1996ao & A 3128 & $\mathrm{Ia}^{\dagger}$ & $0.058^{*}$ & 0.060 & 032803.60 & -525353.6 & $1.8 \mathrm{E} 0.8 \mathrm{~S}$ & $02 / 08 / 96$ \\
\hline SN 1996ap & A3806 & nIa & $0.075^{*}$ & 0.076 & 214553.49 & -570827.3 & $8.6 \mathrm{E} 4.0 \mathrm{~N}$ & $11 / 08 / 96$ \\
\hline SN 1996bm & A2819 & nIa & 0.164 & 0.075 & 004440.85 & -634444.6 & $0.3 \mathrm{~W} 0.6 \mathrm{~N}$ & $04 / 10 / 96$ \\
\hline SN 1996bx & A 3202 & $\mathrm{Ia}^{\dagger}$ & 0.058 & 0.069 & 035916.45 & -532226.3 & $0.8 \mathrm{E} 0.2 \mathrm{~N}$ & $18 / 11 / 96$ \\
\hline SN 1997by & A1736 & $\mathrm{Ia}^{\dagger}$ & $0.045^{*}$ & 0.046 & 132727.54 & -271921.7 & $7.0 \mathrm{~W} 6.6 \mathrm{~N}$ & $27 / 04 / 97$ \\
\hline SN 1997bz & A1238 & $\mathrm{Ia}^{\dagger}$ & 0.030 & 0.072 & 112225.46 & +011121.5 & $0.0 \mathrm{E} 0.2 \mathrm{~S}$ & $27 / 04 / 97$ \\
\hline SN 1997cl & A3577 & $\operatorname{IIn}^{\dagger}$ & $0.047^{*}$ & 0.050 & 135418.71 & -274427.9 & $2.5 \mathrm{~W} 3.9 \mathrm{~N}$ & $02 / 05 / 97$ \\
\hline SN $1997 \mathrm{~cm}$ & A 3528 & nIa & 0.071 & 0.053 & 125337.03 & -284848.5 & $3.4 \mathrm{E} 0.5 \mathrm{~N}$ & $02 / 05 / 97$ \\
\hline SN 1997cp & A3806 & Ia? & 0.160 & 0.076 & 214436.50 & -572530.4 & $2.3 \mathrm{~W} 1.3 \mathrm{~N}$ & $02 / 06 / 97$ \\
\hline SN 1997cr & A3744 & $\mathrm{II}^{\dagger}$ & 0.077 & 0.038 & 210548.21 & -251329.8 & $0.1 \mathrm{E} 0.6 \mathrm{~S}$ & $02 / 06 / 97$ \\
\hline SN 1997cu & A 3128 & $\mathrm{Ia}^{\dagger}$ & $0.062 *$ & 0.060 & 032903.05 & -524141.1 & 0.7E 0.9S & $04 / 07 / 97$ \\
\hline SN $1997 \mathrm{cv}$ & A3565 & nIa & 0.024 & 0.012 & 133520.77 & -341211.6 & $7.6 \mathrm{~W} 9.6 \mathrm{~N}$ & $07 / 07 / 97$ \\
\hline SN 1997cy & A3266 & $\operatorname{IIpec}^{\dagger}$ & $0.063^{*}$ & 0.059 & 043254.81 & -614257.5 & $0.9 \mathrm{E} 1.4 \mathrm{~N}$ & $16 / 07 / 97$ \\
\hline SN 1997cz & A3565 & nIa & 0.048 & 0.012 & 133709.39 & -334731.9 & $2.0 \mathrm{E} 4.4 \mathrm{~N} 2$ & $19 / 07 / 97$ \\
\hline SN 1997df & A 3223 & nIa & $0.059^{*}$ & 0.060 & 040913.80 & -304223.2 & $5.5 \mathrm{~W} 2.9 \mathrm{~N}$ & $08 / 09 / 97$ \\
\hline SN 1997dr & A3112 & Ia? & $0.075^{*}$ & 0.075 & 031849.21 & -442813.4 & $0.2 \mathrm{E} 0.5 \mathrm{~N}$ & $07 / 11 / 97$ \\
\hline SN $1997 \mathrm{fb}$ & A3301 & $\mathrm{Ia}^{\dagger}$ & $0.053^{*}$ & 0.054 & 050114.60 & -383812.7 & $1.3 \mathrm{~W} 0.4 \mathrm{~N}$ & $31 / 12 / 97$ \\
\hline SN $1997 \mathrm{fc}$ & A 3301 & $\mathrm{Ia}^{\dagger}$ & $0.054^{*}$ & 0.054 & 045958.81 & -385504.3 & $4.9 \mathrm{E} 1.9 \mathrm{~N}$ & $31 / 12 / 97$ \\
\hline SN 1997fd & A 3158 & Ia? & 0.190 & 0.059 & 034115.65 & -532930.8 & $7.1 \mathrm{E} 1.3 \mathrm{~S}$ & $28 / 12 / 97$ \\
\hline SN 1997fe & A 3266 & nIa & $0.059^{*}$ & 0.059 & 043307.60 & -612619.2 & 5.7E $6.2 \mathrm{~S}$ & $31 / 12 / 97$ \\
\hline SN 19980 & A3566 & nIa & $0.049^{*}$ & 0.050 & 133934.98 & -354632.5 & $1.1 \mathrm{E} 16.4 \mathrm{~N}$ & $27 / 01 / 98$ \\
\hline SN 1998P & A3566 & nIa & 0.097 & 0.050 & 133717.63 & -352732.6 & $2.3 \mathrm{E} 2.5 \mathrm{~N}$ & $27 / 01 / 98$ \\
\hline SN 1998Q & A3562 & nIa & 0.040 & 0.050 & 133246.38 & -313723.4 & $0.1 \mathrm{E} 1.5 \mathrm{~N}$ & $27 / 01 / 98$ \\
\hline SN $1998 Z$ & A3341 & $\mathrm{Ia}^{\dagger}$ & $0.038^{*}$ & 0.038 & 052458.78 & -312138.1 & $1.5 \mathrm{~W} 2.3 \mathrm{~S}$ & $16 / 03 / 98$ \\
\hline SN 1998bq & A2029 & nIa & 0.095 & 0.077 & 151024.94 & +055530.0 & $1.2 \mathrm{~W} 1.7 \mathrm{~S}$ & $18 / 04 / 98$ \\
\hline SN 1998br & A2029 & Ia? & $0.081^{*}$ & 0.077 & 151020.53 & +055116.5 & $5.1 \mathrm{E} 3.6 \mathrm{~N}$ & $28 / 04 / 98$ \\
\hline SN 1998bs & A 3558 & nIa & $0.047^{*}$ & 0.048 & 132631.51 & -310908.9 & $0.1 \mathrm{E} 0.5 \mathrm{~N}$ & $18 / 04 / 98$ \\
\hline SN 1998bt & A1736 & nIa & $?$ & 0.046 & 132541.93 & -264655.7 & - & $10 / 03 / 98$ \\
\hline SN 1998ch & A3559 & $\mathrm{II}^{\dagger}$ & $0.047 *$ & 0.046 & 132913.31 & -291726.8 & $1.3 \mathrm{~W} 1.8 \mathrm{~S}$ & $18 / 05 / 98$ \\
\hline SN $1998 \mathrm{~cm}$ & A1780 & $\mathrm{Ia}^{\dagger}$ & $0.080^{*}$ & 0.079 & 134412.97 & +030040.3 & $0.2 \mathrm{E} 0.4 \mathrm{~N}$ & $10 / 06 / 98$ \\
\hline SN 1998cq & A2819 & nIa & 0.150 & 0.075 & 004506.11 & -634950.4 & $0.3 \mathrm{E} 1.2 \mathrm{~N}$ & $17 / 06 / 98$ \\
\hline SN 1998do & A168 & Ia? & 0.092 & 0.045 & 011430.92 & +002813.0 & $0.2 \mathrm{E} 0.4 \mathrm{~N}$ & $01 / 08 / 98$ \\
\hline SN 1998dp & A2401 & nIa & $0.057^{*}$ & 0.057 & 215828.12 & -195805.9 & $0.0 \mathrm{E} 0.6 \mathrm{~N}$ & $14 / 08 / 98$ \\
\hline SN 1998du & A2819 & nIa & $0.076^{*}$ & 0.075 & 004511.73 & -634821.6 & $1.9 \mathrm{E} 0.8 \mathrm{~N}$ & $14 / 08 / 98$ \\
\hline SN 1998dv & A3266 & Ia? & 0.155 & 0.059 & 042946.79 & -613025.4 & $2.1 \mathrm{~W} 0.3 \mathrm{~N}$ & $22 / 08 / 98$ \\
\hline SN 1998dw & A151 & $\mathrm{Ia}^{\dagger}$ & $0.049^{*}$ & 0.053 & 010911.56 & -152946.7 & $2.7 \mathrm{E} 1.1 \mathrm{~N}$ & $28 / 08 / 98$ \\
\hline SN 1998dy & A3266 & nIa & $?$ & 0.059 & 042954.24 & $-61 \quad 1243.1$ & - & $14 / 07 / 98$ \\
\hline SN 1998dz & A 3122 & $\mathrm{Ia}^{\dagger}$ & 0.091 & 0.064 & 032058.18 & -410522.8 & - & 03/09/98 \\
\hline SN 1998ea & A3266 & $\mathrm{Ia}^{\dagger}$ & $0.057^{*}$ & 0.059 & 043346.59 & -613520.4 & $5.9 \mathrm{~W} 1.5 \mathrm{~N}$ & $06 / 09 / 98$ \\
\hline SN 1998el & A3122 & nIa & 0.100 & 0.064 & 032101.21 & -413306.6 & $0.8 \mathrm{~W} 1.1 \mathrm{~S}$ & $22 / 10 / 98$ \\
\hline SN 1998ez & A 3188 & nIa & $0.072^{*}$ & 0.076 & 035732.93 & -271213.6 & $0.7 \mathrm{E} 4.0 \mathrm{~N}$ & $08 / 12 / 98$ \\
\hline SN 1998fb & A 3128 & Ia? & $0.060^{*}$ & 0.060 & 033045.86 & -523212.2 & $0.3 \mathrm{E} 0.1 \mathrm{~N}$ & $20 / 12 / 98$ \\
\hline SN 1999Y & A3142 & nIa & 0.100 & 0.066 & 033655.81 & -393304.9 & $3.8 \mathrm{~W} 0.9 \mathrm{~S}$ & $10 / 01 / 99$ \\
\hline SN 1999ao & A3392 & $\mathrm{Ia}^{\dagger}$ & $0.055^{*}$ & 0.055 & 062726.37 & -355024.2 & $2.3 \mathrm{~W} 0.9 \mathrm{~N}$ & $05 / 03 / 99$ \\
\hline
\end{tabular}

\subsection{Spectroscopy}

The redshift for a SN is usually measured from a spectrum of the SN which, if taken around maximum light, also distinguishes the SN type (Filippenko 1997). The average discovery magnitude for a $\mathrm{SN}$ in the Mount Stromlo Abell cluster SN search was $m_{V} \sim 19.5$. Taking spectra of objects of this brightness with a $2 \mathrm{~m}$-class telescope is difficult in the best of circumstances, but when the objects are also transient, spectra are difficult to obtain. 
Spectroscopic followup for the Mount Stromlo Abell cluster SN search was primarily taken with the Double Beam Spectrograph on the MSSSO $2.3 \mathrm{~m}$ telescope at a resolution of $4.4 \AA$ A per pixel. Telescope time was scheduled in advance and we were generally awarded 2 nights/month with both the spectrograph and imager mounted simultaneously. This meant that if a new $\mathrm{SN}$ was discovered just after an observing run, it would be a month before a spectrum could be obtained. In general, by that time, the SN would be too faint to spectroscopically type. Many of our SNe fell into this category. Fortunately, the SN community was generous with its spectroscopic time on various telescopes around the world, and several SN typings were obtained through the IAU Circulars from the observations of other astronomers.

For consistency, the redshifts of all the $\mathrm{SNe}$ were determined from spectra of their host galaxies taken with the MSSSO $2.3 \mathrm{~m}$ telescope. The only exceptions were the three $\mathrm{SNe}$ in Table 1 for which we did not detect a host galaxy. All spectra encompassed a wavelength range of $\sim 3900-7500 \AA$, were wavelength calibrated using observations of a $\mathrm{Cu}-\mathrm{He}$ lamp taken at the position of the $\mathrm{SN}$, and flux calibrated using sensitivity curves determined from the observation of southern flux standards chosen from the list of Bessell (1999). The measured SN redshifts, and the redshifts of the target Abell clusters are shown in Table 1. The asterisked $\mathrm{SNe}$ are those which occurred in a galaxy associated with the targeted Abell cluster.

\subsection{Photometry}

As discussed in Paper I, all of the search observations were taken with the $\mathrm{MaCHO}$ wide-field, dual-colour imager on the MSSSO $1.3 \mathrm{~m}$ telescope. This mosaic covered $42^{\prime} \times 42^{\prime}$ and observed simultaneously in the red $\left(R_{\mathrm{M}}\right)$ and blue $\left(V_{\mathrm{M}}\right)$ through non-standard filters (Stubbs et al. 1993; Bessell \& Germany 1999) designed for the MaCHO project. A significant fraction of the SN followup was also taken with this system, since this time was scheduled for every third night and hence less affected than the MSSSO $2.3 \mathrm{~m}$ observations by poor weather. In order to do this, some of the search time on the MSSSO $1.3 \mathrm{~m}$ was sacrificed to take followup observations. Individual clusters with visible $\mathrm{SNe}$ were flagged and their weighting artificially increased (Paper I) so that they were scheduled to be observed every 6 days. Although these data were taken in the non-standard $V_{\mathrm{M}}$ and $R_{\mathrm{M}}$ passbands, they could be incorporated into the standard $B V(R I)_{\mathrm{KC}}$ light curves through $\mathrm{K}$-corrections (Sect. 4.4.2).

Other $B V(R I)_{\mathrm{KC}}$ photometric data, and in particular the photometric calibration data, were taken with the $0.9 \mathrm{~m}$ and $1.5 \mathrm{~m}$ telescopes at the Cerro Tololo Inter-American Observatory (CTIO; see Sect. 4.2).

\section{SN light curves and distances}

As shown in Paper I, image subtraction techniques are now central to the success of modern SN searches. The ability to subtract the light of the host galaxy has revealed many SNe that would have previously remained undiscovered. Subtraction techniques have also improved SN photometry by removing the contaminating light from the host galaxy. This crucial subtraction is, however, only the first step in obtaining the final SN photometry.

\subsection{Relative photometry}

Most of the images for the Mount Stromlo Abell cluster SN search were taken at either Siding Spring Observatory or Mount Stromlo Observatory. Since neither site is renowned for its photometric conditions, the SN magnitudes were determined using a sequence of photometrically calibrated stars in the field of the SN (local standards) to calculate the photometric offset for each frame. This allowed every observation to be used in constructing the SN light curves, regardless of whether or not the night was photometric. To construct a relative light curve for each SN, a procedure similar to that described in Paper I was used.

\subsubsection{The templates}

The photometric followup for the Mount Stromlo Abell cluster $\mathrm{SN}$ search utilised both the MaCHO $V_{\mathrm{M}}, R_{\mathrm{M}}$ system on the MSSSO $1.3 \mathrm{~m}$ telescope and the conventional $B V(R I)_{\mathrm{KC}}$ system on a variety of other telescopes. To avoid colour residuals in the subtractions (Sect. 4.1.3) due to the different bandpasses, different templates were required for each system.

For the MSSSO $1.3 \mathrm{~m}$ data, the template for each SN was selected from the entire database of search observations for the relevant Abell Cluster. To ensure the absence of SN light in the template, pre-discovery observations within one month of discovery were rejected as templates, as were observations taken less than 8 months after discovery. The seeing, sky brightness and transparency of the remaining observations were compared, and the best observation chosen as a template. The $V_{\mathrm{M}}$ and $R_{\mathrm{M}}$ images from this observation were checked to make sure there were no chip defects, cosmic rays or other blemishes that could affect the photometry before being incorporated as the blue and red templates respectively. Unlike the search templates (Paper I), beyond flat-fielding, there was no extra processing of any of these templates.

For the $B V(R I)_{\mathrm{KC}}$ data, no pre-SN images existed. The 4 templates for each field, one in each colour, were created from images taken once the SN had faded. This allowed the light from the host galaxy to be subtracted without removing any of the SN signal. These templates were created from data taken with the $0.9 \mathrm{~m}$ and $1.5 \mathrm{~m}$ telescopes at CTIO, at least 8 months after the discovery of the SN. Each template was created using an image combination method incorporating the AUTOCLEAN program developed by J. Tonry. In this technique, the images are added together to form a combined image, after being scaled to the same level and flux matched. To eliminate cosmic rays, the program looks for a gradient in the local sky brightness (which would indicate a star or galaxy) and interpolates over any sharp variations (cosmic rays). The cosmic ray rejection process works best with 3 or more images and was not found to reject the centres of unsaturated stars. 


\subsubsection{Image registration}

A more robust method of image registration than that used in Paper I was required to align the followup images with their templates. Only one template image per filter was created for each $\mathrm{SN}$, which meant that we could not fix the image orientation relative to the template. Also, since the $B V(R I)_{\mathrm{KC}}$ data was taken with different instruments on different telescopes, it was also not possible to constrain the pixel scale for this data.

In this revised registration method, the SourceExtractor artificial neural network (Bertin \& Arnouts 1996) was used to identify stars in the template and each image. The star matching algorithm of Groth (1986) was then used to identify similar triangles and each triangle was fit with a linear transformation of the form:

$x_{2}=a x_{1}+b y_{1}+c$

$y_{2}=d x_{1}+e y_{1}+f$.

The algorithm then looked for values of $a, b, c, d, e$ and $f$ constrained in a small region of this 6 parameter space to determine the correct registration. Typically 50 stars at a time were used from the template and the image. If no match was found, the next 50 stars were chosen and so on, until all stars had been compared to all others. The advantage of this method is that it robustly matches images of different rotation and scale provided there is a large region of overlap (as is the case here). However, this method does not work so well if one has a wide-field image and small-field image. In this case, more than 50 stars must be compared at a time (since the 50 stars chosen in the small-field image by SourceExtractor may only correspond to 2 stars in the wide-field image) and the algorithm scales as $N^{3}$.

\subsubsection{Image subtraction}

In Paper I we described how, after each search image was registered to the template, the template was convolved and photometrically scaled to match the atmospheric and photometric conditions of the registered image. This procedure allowed the template to be directly subtracted from the registered search image. While usually producing a good subtraction, the quality of the subtraction depends greatly on the quality of the kernel determined from isolated bright stars in the field.

A better method, in most cases, is the optimised subtraction process developed by Alard (2000). By decomposing the kernel into a series of functions, it is possible to convert a nonlinear least-squares problem into a linear least-squares problem which is easily solved to return the background, scale and optimal kernel for each image. The main advantage of this method over the one used in Paper I is that it accounts for PSF variations across the field - an important consideration when dealing with the MSSSO $2.3 \mathrm{~m}$ data.

The aim of the subtraction process is to create an image in which all the light from the host galaxy is removed, leaving only the flux of the SN. This is achieved by replacing a small region of the shifted image centred on the SN, with the equivalent region from the subtracted image which has been scaled so that the backgrounds in the two images match. With this technique, the light from the host galaxy is effectively removed from the shifted image and substituted with the SN, allowing the photometry of the SN and the local standard stars to be performed simultaneously.

In addition, to determine an appropriate error for the measured SN magnitude, $\sim 10$ stars with the same magnitude as the $\mathrm{SN}$ were added to galaxies and blank sky around the position of the $\mathrm{SN}$ in the shifted image. The template was then resubtracted from the shifted image and, as for the SN, regions centred on the positions of each of these added stars were replaced with the equivalent regions from the background scaled, subtracted image. The shifted image with the appropriate substitutions was then photometered to derive instrumental magnitudes and fluxes for the SN, the local standard stars and the added stars.

\subsubsection{Measuring the photometry}

Both aperture and PSF photometry were performed for each shifted image using IRAF tasks APPHOT and DoPHOT (Schechter et al. 1993) respectively. The aperture and PSF photometry for the $\mathrm{SN}$, the local standard stars and the added stars were calculated simultaneously and recorded in flux as well as magnitudes. Although PSF photometry works much better for faint objects such as $\mathrm{SNe}$, it is much more complicated than simple aperture photometry and more prone to returning incorrect photometry in a semi-automated routine such as was developed for this project. The most common problem encountered with the PSF photometry was incorrect photometry resulting from a poorly constructed PSF. If the PSF is not close to that of the data (if a saturated star or a star in a defective section of the CCD is included in the creation of the PSF), the returned photometry will not be correct. Therefore, although the PSF photometry was used to create the final SN light curves, the aperture photometry was used to first check that the PSF photometry was working as expected.

One other problem encountered with the PSF photometry from DoPHOT, arose in images taken before the SN was detected and after the SN had faded. The observation of the field taken immediately before the observation in which the SN was discovered, and the first observation where the SN was no longer visible, were used to obtain upper limits to help constrain the template fits (Sect. 4.4). On a few occasions, however, DoPHOT did not converge to a measurement, and for these non-detections, the magnitudes, fluxes and errors derived from aperture photometry were used.

\subsection{Calibrated photometry}

The above photometry gives the instrumental magnitudes of the SN, the added stars and the local standard stars. However, in order to derive calibrated magnitudes for the $\mathrm{SN}$, it is necessary to calibrate the magnitudes of the local standards.

The photometric observations required to calibrate the local standard stars were taken during an 8 month visit to CTIO with extensive use of the $0.9 \mathrm{~m}$ and $1.5 \mathrm{~m}$ telescopes. During this time, 21 of the nights scheduled for the Mount Stromlo 
Table 2. Averaged colour terms for the CTIO data.

\begin{tabular}{cccc}
\hline \hline Magnitude & Colour term & Mean error & Colour index \\
\hline \multicolumn{4}{c}{ CTIO 0.9 m Tek 3k_3 (7 nights) } \\
\hline$B$ & 0.092 & 0.014 & $B-V$ \\
$V$ & -0.016 & 0.002 & $B-V$ \\
$R$ & -0.007 & 0.007 & $V-I$ \\
$I$ & -0.020 & 0.003 & $V-I$ \\
\hline \multicolumn{5}{c}{ CTIO 0.9 m Site 2k_6 (6 nights) } \\
\hline$B$ & 0.094 & 0.003 & $B-V$ \\
$V$ & -0.018 & 0.005 & $B-V$ \\
$R$ & -0.007 & 0.006 & $V-I$ \\
$I$ & -0.015 & 0.004 & $V-I$ \\
\hline \multicolumn{5}{c}{ CTIO 1.5 m Site2k_6 (8 nights) } \\
\hline$B$ & 0.095 & 0.012 & $B-V$ \\
$V$ & -0.034 & 0.013 & $B-V$ \\
$R$ & -0.005 & 0.006 & $V-I$ \\
$I$ & -0.013 & 0.005 & $V-I$ \\
\hline
\end{tabular}

Abell cluster SN search were photometric and used in the calibrations. On each photometric night, Landolt standard fields (Landolt 1992) spanning a large range of airmass $(X \sim 1.07$ to $X \sim 2.1)$ and colour $(B-V \sim-0.3$ to $B-V \sim 1.9)$ were observed, in addition to some fraction of the $48 \mathrm{SN}$ fields in the sample. To determine accurately the colour terms and atmospheric extinction correction for each night, a Landolt standard field was observed approximately every half hour resulting in $\sim 15$ fields per night. Spacing the observations in this way also allowed the photometric quality of the night to be monitored.

The local standard stars in the field of each SN were selected by eye to be uncrowded and sufficiently bright for accurate photometry. Between 5 and 23 local standards were chosen for each SN field depending on the crowding of the field.

PSF photometry for the local standard stars was produced by processing all the calibration frames with DAOPHOT (Stetson 1987). As suggested in Harris et al. (1981), each of the 21 nights were reduced independently to solve for the nightly colour terms. The colour terms for each CCD/telescope combination were then averaged across all runs and used, with the nightly extinction corrections, to re-reduce the data to final photometry. The average colour terms calculated for the CTIO CCD/telescope combinations are listed in Table 2 with the sense that $v=a_{0}+V-0.016(B-V)+a_{1} \times X$ for the CTIO $0.9 \mathrm{~m}+$ Tek $3 \mathrm{k} 3$ as an example. Here, $a_{0}$ and $b_{0}$ are the coefficients to the fit, $X$ is the airmass, $v$ is the instrumental magnitude and $V$ is the absolute magnitude of the stars.

Since the local standard stars were selected based solely on proximity to the $\mathrm{SN}$, some of them were variable, and not useful as local standards. These stars were filtered out of the final photometry list by looking at the root-meansquare of the ratio of the observed observation-to-observation scatter and the expected observation-to-observation scatter based on the input error estimates for each star in each filter. Stars with a large ratio $(>2)$ were examined and those stars that appeared variable rejected. Stars with only one observation in all 4 colours were also rejected since there was insufficient information to check for variability.
Finding charts for each field are presented at the EDP Sciences website (EDP: http://www.edpsciences.org/) and the local standard star photometry in $B V(R I)_{\mathrm{KC}}$ are available from the Strasbourg astronomical Data Centre (CDS: http://cdsweb.u-strasbg.fr/cgi-bin/qcat?J/A+A/415/863).

For the $B V(R I)_{\mathrm{KC}}$ data, DAOPHOT and its associated programs output the final calibrated photometry for the local standard stars. For the MaCHO $V_{\mathrm{M}}, R_{\mathrm{M}}$ data, however, further calibrations were required to transform the $B V(R I)_{\mathrm{KC}}$ magnitudes into $V_{\mathrm{M}}$ and $R_{\mathrm{M}}$ magnitudes. The transformation equations were determined by measuring the slope of the regressions to the data presented in Bessell \& Germany (1999). They are:

$V_{\mathrm{M}}=0.153(B-V)+V$

$R_{\mathrm{M}}=-0.154(V-I)+R$

valid over a range of -0.13 to 1.12 in $(B-V)$ and -0.11 to 1.13 in $(V-I)$. The error in each transformation was determined by calculating the $V_{\mathrm{M}}$ and $R_{\mathrm{M}}$ magnitudes for the local standard stars from their calibrated $B V(R I)_{\mathrm{KC}}$ magnitudes using the above equations, and comparing these calculated magnitudes to the actual $V_{\mathrm{M}}$ and $R_{\mathrm{M}}$ magnitudes measured by the telescope. This yields an uncertainty of $0.020 \mathrm{mag}$ in the $V_{\mathrm{M}}$ transformation and 0.017 mag in the $R_{\mathrm{M}}$ transformation.

\subsection{Calibrating the $S N$ magnitude}

Once the magnitudes for the local standard stars were calibrated, the calibrated magnitude for the $\mathrm{SN}$ in each observation could be determined. To calibrate each frame, the mean difference between the calibrated photometry of the local standards in the SN field (Sect. 4.2) and the DoPHOT photometry for the local standards on each SN frame (Sect. 4.1.4) was calculated using the robust-mean method developed by Stetson (1996). This offset was then added to the SN magnitude measured by DoPHOT to return the calibrated magnitude of the SN. The fluxes were calibrated in the same way after transforming the calibrated magnitudes of the local standard stars into fluxes. An additional offset of 25 was added so that unit flux was equivalent to 25 th magnitude.

The error in the calibrated SN magnitude was derived from how well DoPHOT was able to recover the fluxes of the added stars. All of the stars added to the shifted image in Sect. 4.1.3 were identical, with the same flux as the $\mathrm{SN}$ in the frame. The added stars underwent the same processing as the SN (i.e. the subtraction and photometry was performed in exactly the same way) and so the dispersion in their recovered fluxes provides an estimate of the error in the $\mathrm{SN}$ measurement. The dispersion in the recovered fluxes was used to determine the uncertainty in the magnitude measurements rather than the dispersion in the recovered magnitudes, since the errors are symmetric in flux space.

To determine the uncertainty in the flux of the $\mathrm{SN}$, the mean flux of the added stars was calculated using the robust-mean technique of Stetson (1996) and the dispersion in the mean used 
to reject $5 \sigma$ outliers. The dispersion of the added stars was then calculated using:

$\sigma_{\text {added }}=\sqrt{\sum_{i} \frac{\bar{f}^{2}-f_{i}^{2}}{N}}$

where $\bar{f}$ is the robust mean for the flux of the added stars calculated using the technique of Stetson (1996), $f_{i}$ is the DoPHOT flux of each of the added stars, and $N$ is the number of added stars. This was used as the error in the DoPHOT flux for the $\mathrm{SN}$. To obtain the uncertainty in the calibrated DoPHOT magnitudes for each $\mathrm{SN}$, this flux error was converted into upper and lower magnitude errors via

magerr $_{-}^{+}=2.5 \log \left(\frac{\text { flux }_{+}^{-} \text {fluxerr }}{\text { flux }}\right)$.

The magnitudes, fluxes and associated errors for each $\mathrm{SN}$ in $B V(R I)_{\mathrm{KC}} V_{\mathrm{M}}$ and $R_{\mathrm{M}}$ are available at the CDS (http://cdsweb.u-strasbg.fr/cgi-bin/qcat?J/A+A/415/863).

The next step is to turn these calibrated $\mathrm{SN}$ magnitudes, measured in up to six different filters, into a distance to the SN. There are several different methods by which the SN distance can be measured - we have chosen to elaborate on the simplest of these, the $\Delta m_{15}$ method.

\section{4. $\Delta m_{15}$ revisited}

The $\Delta m_{15}$ distance indicator was pioneered by Phillips (1993), and was the first method to accurately account for the effects of light curve shape in measuring $\mathrm{SN}$ distances. This first implementation simply measured how fast a SN fades in the $B$ band over the 15 days following maximum light. However, to measure $\Delta m_{15}$ on noisy data, it is necessary to define a set of templates where the data are sufficiently good that $\Delta m_{15}$ can be measured directly from the data. These templates are then used to fit a given $\mathrm{SN}$, with the range of acceptable templates defining the range of acceptable $\Delta m_{15}$ (Hamuy et al. 1996a; Phillips et al. 1999).

A complete reconstruction of the $\Delta m_{15}$ method was required to address several features of the photometric data from the Mount Stromlo Abell cluster SN search: 1) a substantial portion of the dataset is $R$ band data for which a $\Delta m_{15}$ relation has not been determined, 2) most SNe from the Mount Stromlo Abell cluster SN search have only sparsely sampled data and hence non-detections and data with low $\mathrm{S} / \mathrm{N}$ were required to constrain the template fits, and 3) a significant fraction of the data was obtained through non-standard filters and many $\mathrm{SNe}$ were discovered at redshifts beyond $z>0.05$, requiring the implementation of large K-corrections.

To address point one, we apply the same techniques as Phillips et al. (1999) to create a $\Delta m_{15}$ vs. calibrated magnitude relation for the $R$ band data (Sect. 4.4.4). Points two and three have a lot in common with the high-redshift SN data. Perlmutter et al. (1997) advocate making flux (rather than magnitude) measurements and fitting the template light curves to the SN data (rather than correcting the data to the rest frame and fitting the data to the templates). This philosophy is adopted in
Sect. 4.4.2 in the context of our implementation of the $\Delta m_{15}$ method. Finally, since many of the Mount Stromlo Abell cluster SN search objects are beyond $z>0.05, \mathrm{~K}$-corrections are not negligible. This is further exacerbated due to the large fraction of SN data taken through non-standard passbands which must be transformed back to standard $B V(R I)_{\mathrm{KC}}$ through K-corrections. Nugent et al. (2002), Riess et al. (1998) and Perlmutter et al. (1999) suggest iteratively fitting K-corrections within the distance fitting procedure (providing a better estimate of $t_{0}^{B}$ ), and applying corrections to the spectra based on the actual colour evolution of the $\mathrm{SN}$ in question. This procedure is also adopted in Sect. 4.4.2.

The following sections describe in detail our implementation of the $\Delta m_{15}$ method and its use in determining the distances to the SNe. Section 4.4.1 describes the creation of the template light curves which are used in Sects. 4.4.2 and 4.4.3 to determine the $t_{0}^{B}, B_{\max }, V_{\max }, R_{\max }$ and $I_{\max }$ for each $\mathrm{SN}$. These values are used in Sect. 4.4.4 to determine the reddening-free luminosity-decline rate relations in $B, V, R$ and $I$ which are required in Sect. 4.4.3 to determine the distances to the $\mathrm{SNe}$.

\subsubsection{Constructing the template light curves}

Hamuy et al. (1996a) constructed a set of six template light curves in $B, V$ and $I$ to analyse the SN data taken during the Calán/Tololo Search. However, followup observations for the Mount Stromlo Abell cluster SN search were also obtained in $R$ and so template light curves needed to be constructed for $R$ band data.

We constructed template light curves in $B, V, R$ and $I$ for five of the six SNe used by Hamuy et al. (1996a): 1991T (Lira et al. 1998), 1991bg (Filippenko et al. 1992; Leibundgut et al. 1993; Turatto et al. 1996), 1992A (Suntzeff, private communication), 1992bc and 1992bo (Hamuy et al. 1996b). In addition, we have chosen several other well-observed objects to increase the number of templates to $16: 1990 \mathrm{~N}$ (Lira et al. 1998), 1994D (Smith, private communication), 1994ae, 1995D, 1995E, 1995ac, 1995al, 1995bd, 1996X, 1996bo (Riess et al. 1999a), and 1998bu (Jha et al. 1999a; Suntzeff et al. 1999).

Our templates were constructed by fitting a spline to each SN's light curve, selecting the knots of the spline by hand, and minimising the residual of the spline to the data set. A direct minimisation of the $\chi^{2}$ value could not be used to easily decide an appropriate model, since splines ring if the shape of the fit is complicated, there are large gaps between the data points, and too many knots are chosen. The best fit to the data was taken as the one that had a low chi-squared value and expressed the nuances of the actual data but did not ring. Once an acceptable fit was found, the time of $B$ maximum, $t_{0}^{B}$, for each $\mathrm{SN}$ was taken as the maximum in $B$ spline fit, and the spline fits for each colour were $\mathrm{K}$-corrected back to the rest-frame and corrected for time-dilation (see Sect. 4.4.2). $\Delta m_{15}$ for each template was then defined by the magnitude difference between the maximum in the $B$ spline, and that of the fitted spline, 15 days later.

Following the prescription in Hamuy et al. (1996a), the peak magnitudes were normalised so that $B=V=R=I=$ $0.0 \mathrm{mag}$ at $t_{0}^{B}$. As a final step, each template light curve was 
extended to $\sim 20$ days before $B_{\max }$ using the rise-time prescription of Riess et al. (1999b). This extrapolation was assigned an uncertainty of $\sim 20 \%$, and was useful in constraining the template fits to the $\mathrm{SN}$ data in Sect. 4.4.2, especially in cases where we had non-detections, or marginal detections before discovery.

Comparison with Phillips et al. (1999) indicates our two methods agree to a rms of $0.05 \mathrm{mag}$ in the measure of $\Delta m_{15}$ with one exception, SN 1995ac. This SN, which has a $\Delta m_{15}=$ 0.69 by our method, and $\Delta m_{15}=1.0$ according to Phillips et al. (1999), has a very flat evolution near maximum, making the exact date of $t_{0}^{B}$, and hence $\Delta m_{15}$, uncertain. This highlights the main difficulty in constructing the templates and in the $\Delta m_{15}$ method itself - if the $t_{0}^{B}$ is shifted by an interval as small as half a day - the $\Delta m_{15}$ can change substantially. In all that follows, SN 1995ac has not been included as a template, because of this uncertainty.

\subsubsection{K-correction to rest-frame magnitudes}

SN magnitudes are always measured in the observer's frame with a particular filter. However, to derive accurate SNIa distances using $\Delta m_{15}$, these observations must be corrected to restframe $B V(R I)_{\mathrm{KC}}$. In addition, observations taken through non-standard filters (in this case the $V_{\mathrm{M}}$ and $R_{\mathrm{M}}$ filters) must also be transformed to standard $B V(R I)_{\mathrm{KC}}$ light curves. These redshift and filter transformations are incorporated through K-corrections.

The single-filter K-correction was defined by Oke \& Sandage (1968) and used by Hamuy et al. (1993a) to calculate SNIa K-corrections in the $B$ and $V$ bandpasses. With the discovery of distant $(z>0.5) \mathrm{SNe}$, Kim et al. (1996) developed a more general K-correction which allows nearby and distant SN magnitudes measured in different filters to be compared. Equation (8) calculates the correction required to transform the magnitude of an object with spectrum $F(\lambda)$ observed in a filter with sensitivity function $S_{i}(\lambda)$, to the corresponding magnitude in a filter with sensitivity function $S_{j}(\lambda)$

$K_{i j}=2.5 \log \left[(1+z) \frac{\int F(\lambda) S_{i}(\lambda) \mathrm{d} \lambda}{\left.\int F(\lambda /(1+z)) S_{j}(\lambda) \mathrm{d} \lambda\right)}\right]+Z_{j}-Z_{i}$.

The ability to K-correct between different filters allows magnitudes measured in non-standard passbands to be converted to rest-frame magnitudes provided the sensitivity functions of the non-standard filters are known. This enables the $V_{\mathrm{M}}$ and $R_{\mathrm{M}}$ data taken during the Mount Stromlo Abell cluster SN search to be $\mathrm{K}$-corrected to standard, rest-frame $B V(R I)_{\mathrm{KC}}$, and has prompted the development of specialised filters designed to maximise sensitivity at certain redshifts (Schmidt et al. 1998).

The K-corrections from $V_{\mathrm{M}}$ to $V^{(z=0)}$ and $V_{\mathrm{M}}$ to $B^{(z=0)}, R_{\mathrm{M}}$ to $R_{\mathrm{KC}}^{(z=0)}$ and $R_{\mathrm{M}}$ to $I_{\mathrm{KC}}^{(z=0)}$ and redshifted $B V(R I)_{\mathrm{KC}}$ to rest-frame $B V(R I)_{\mathrm{KC}}$ for each SNIa redshift were calculated using Eq. (8). The series of SNIa spectra in Hamuy et al. (1993a) with the addition of SN 1994D spectra (Filippenko 1997) were used in the calculation. The $B V(R I)_{\mathrm{KC}}$ sensitivity functions were taken from Bessell (1990) and the sensitivity functions determined in Bessell \& Germany (1999) were used for the $V_{\mathrm{M}}$ and $R_{\mathrm{M}}$

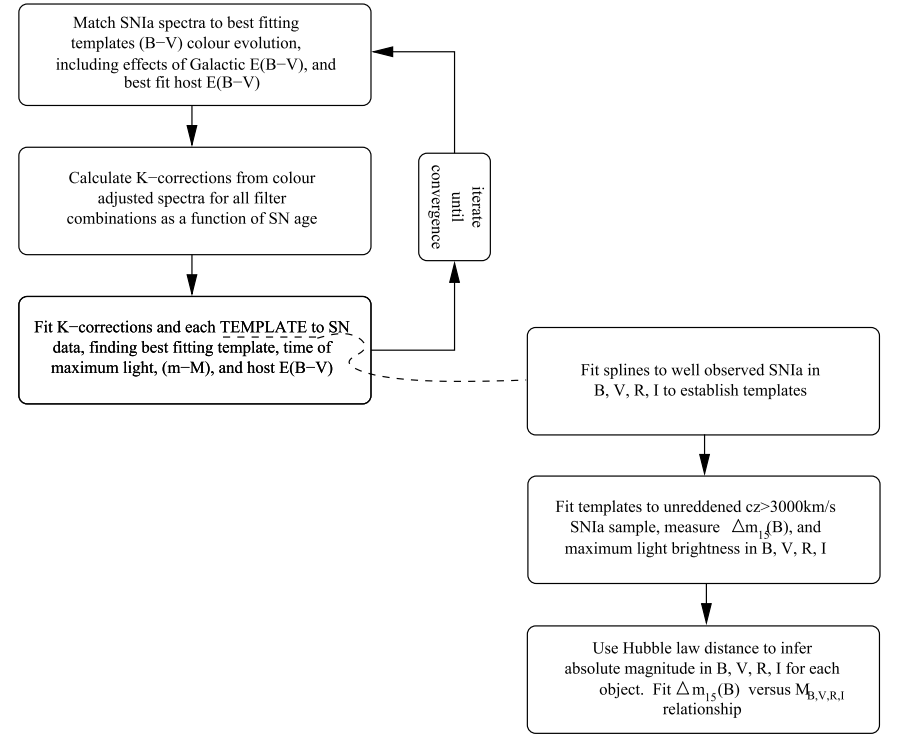

Fig. 1. Flowchart showing iterative procedure for template fitting.

filters. The resulting $\mathrm{K}$-corrections, which span from 15 days before $B_{\max }$ to 80 days after $B_{\max }$, were fit with a least-squares spline (knots predefined at $-15,-5,0,5,15,30$, and 80 days relative to maximum) to smoothly provide $\mathrm{K}$-corrections for arbitrary epochs. The uncertainty is gauged from the rms scatter of individual spectra around the fitted spline.

To apply the correct K-corrections to SN data, one must know the age of the SN. In addition, two colour-dependent complications haunt SNIa K-corrections: 1) extinction affects the spectral energy distribution of the SN and 2) the colours of SNIa at a given age are not homogeneous, but related to the absolute magnitude of the SN (e.g. Phillips et al. 1999; Riess et al. 1996). These have a significant effect on the $\mathrm{K}$-corrections and must be included in the calculation (Nugent et al. 2002). Because of these complications, instead of applying the $\mathrm{K}$-corrections to the $\mathrm{SN}$ data, a better method is to invert the process, applying K-corrections to the templates, to transform these $z=0$ light curves (with accurate colour evolution and time of maximum light pre-defined) to the observed redshift of each SN. The templates are then matched to the data in the observer's frame, rather than the restframe.

Before measuring the K-corrections, the spectra are corrected to match the colour evolution of the template, including the current best fit value for extinction, and the value of Galactic reddening. For purposes of the K-corrections, the colours of a given spectrum are made to match the desired $B-V$ colour by applying the Savage \& Mathis (1979) reddening law to the entire spectrum (either adding or subtracting reddening) as suggested by Nugent et al. (2002).

\subsection{3. $\mathrm{SN}$ fitting procedure}

To match a $\mathrm{SN}$ to a template, we use the iterative procedure schematically depicted in Fig. 1.

The first step is to estimate K-corrections. For iteration one, this is done by assuming zero host extinction, a 
colour evolution given by the Leibundgut template (thesis, Basel University), and using a value of $E(B-V)_{\text {gal }}$ from Schlegel et al. (1998). For subsequent iterations the best template with its best fitted value of host extinction is used. Given these parameters, each spectrum is matched to the expected $(B-V)$ colour as described above, and the $\mathrm{K}$-corrections calculated for each filter applied to the 15 template light curves. These template lightcurves are fit to the data through a $\chi^{2} \mathrm{~min}-$ imisation,

$$
\begin{aligned}
\chi^{2} & =\sum_{\text {filterfromto }} \\
& \sum_{\text {obs }} \frac{\left[\operatorname{mode}_{\text {from }}(t)-\text { data }_{\text {from }}(t)\right]^{2}}{\left(\sigma_{\text {template }}^{2}\left(t-t_{0}\right) /(1+z)+\sigma_{\text {data }}^{2}(t)+\sigma_{\mathrm{KC}}^{2}\left(t-t_{0}\right) /(1+z)\right.}
\end{aligned}
$$

where

$$
\begin{aligned}
\text { model }_{\text {from }}(t)= & \text { template }_{\mathrm{to}}\left(\frac{t-t_{0}}{1+z}\right)+M_{\mathrm{to}}\left(\Delta m_{15}\right) \\
& +K_{\text {from-to }}\left(\frac{t-t_{0}}{1+z}\right)+A_{\mathrm{to}} E(B-V)_{\mathrm{host}} \\
& +A_{\text {from }} E(B-V)_{\mathrm{gal}}+(m-M) .
\end{aligned}
$$

Here we are transforming a series of observer frame observations, denoted as "from" filters, to a corresponding rest frame filter, the "to" filter. $A_{\text {from }}$ and $A_{\text {to }}$ is the extinction value per magnitude of $E(B-V)$ for the "from" and "to" filters. To determine the best-fit template, the magnitude in the rest frame $\left(m_{\mathrm{to}}\right)$ for each bandpass, the host galaxy extinction $A_{\mathrm{to}} E(B-V)_{\text {host }}$ and the time of maximum $\left(t_{0}\right)$ were chosen so that $\chi^{2}$ was minimised. $M_{\mathrm{to}}\left(\Delta m_{15}\right)$ is the unreddened absolute magnitude of the SN template (based on its $\Delta m_{15}$ ) at maximum light in the "to" filter, and this relationship is estimated in Sect. 4.4.4.

The errors in $t_{0}^{B}, B_{\max }, V_{\max }, R_{\max }$ and $I_{\max }$ for each $\mathrm{SN}$ were estimated by varying the value of each parameter individually and marginalising over all other variables. The error was then derived from the range of fits for that variable with $\chi^{2}<\chi_{\min }^{2}+1$. In all cases, the $\chi^{2}$ were renormalised such that $\chi_{\min }^{2} /$ d.o.f. $=1$ (Press et al. 1994). The error in $\Delta m_{15}$ was taken as the range of the $\Delta m_{15}$ measurements for all the templates whose $\chi^{2}$ fit to the SN data was within $1 \sigma$ of the $\chi^{2}$ of the best fit. To this, an additional error of $0.1 \mathrm{mag}$, representing the intrinsic dispersion of our $\Delta m_{15}$ fitting method, was added in quadrature for those SNe with $0.9<\Delta m_{15}<1.45$, and an error of 0.2 added in quadrature for those SNe with $\Delta m_{15}$ outside this range. The larger additional error for the fast and slow decliners arises because these regions are only poorly sampled by the templates. Of the 15 templates used, 10 have a $\Delta m_{15}$ between 0.9 and 1.45 , only 2 have $\Delta m_{15}>1.45$ and 3 have $\Delta m_{15}<0.9$.

In practice, on real-world data, the derived value of $E(B-V)_{\text {host }}$ can be negative, which does not indicate that the $\mathrm{SN}$ is "blue-ened", but rather indicates either an allowable fluctuation due to noise, or a departure from the mean $\Delta m_{15}$ relation by the object (as is probably the case for SN 1994D). The noise fluctuation may be remedied by applying a "Baysian filter", with an a priori extinction distribution, $p\left(A_{V}\right)$, of a onesided Gaussian with a maximum at $A_{V}=0$ and $\sigma\left(A_{V}\right)=1 \mathrm{mag}$ (Riess et al. 1996; Jha et al. 1999) to the $E(B-V)_{\text {host }}$ of each SN.
The second situation is difficult to pinpoint, especially in the sparsely sampled data of the Mount Stromlo Abell cluster SN search. Although applying a Baysian correction effectively ignores the possibility of a departure from a one-parameter brightness-decline relation, due to the poor quality of much of the data, any real "blue-ing" would be hidden by the uncertainties. We apply the Baysian filter to the data, but acknowledge that this is not the correct thing to do to correct for intrinsic colour variation of SNIa.

The best fit value for $E(B-V)$ host was then used, in combination with the colour evolution of the best template (time zero pointed using the best fit time of maximum light), to colour match the spectra used in the K-corrections. The whole fitting procedure was iterated with all 15 templates until convergence, and returned the distance modulus, $(m-M)$, and the $E(B-V)_{\text {host }}$ to each SN.

Once again, the errors in $(m-M)$ and $E(B-V)_{\text {host }}$ were estimated by varying each parameter individually and marginalising over all other variables and all templates. The error was derived from the range of fits for that variable with $\chi^{2}<\chi_{\min }^{2}+1$. This error provides for the statistical uncertainty in the measurement due to photometric noise, and template errors, however, it does not include the intrinsic uncertainty in our $\Delta m_{15}$ method.

The SNIa and untyped SNe from the Mount Stromlo Abell cluster SN search, as well as 61 of the SNe analysed by Phillips et al. (1999), were processed in this manner. However, the $\Delta m_{15}$ technique is only valid for SNIa. Unfortunately, most of the $\mathrm{SNe}$ discovered in the Mount Stromlo Abell cluster SN search were not spectroscopically typed (see Sect. 3.1) which meant that their typing had to be determined photometrically.

To select the SNIa from the sample, each untyped SN was classified as SNIa or not based on the goodness of fit of the best fitting template light curve. This was done by calculating a $\chi^{2}$ value for each of the template fits to the data for each SN, and visually inspecting the fit with the lowest $\chi^{2}$ value. Simply taking the $\mathrm{SNe}$ with low $\chi^{2}$ values as the SNIa is not a valid way of extracting the SNIa from the data set, since each SN has a different number of data points and is not necessarily observed in all colours. In addition, since the shape of the light curve is more complicated at early times, it is more important to fit the data well at these epochs. For this reason, regardless of the numerical value for $\chi^{2}$, if the template appeared to fit the data well in all colours, the SN was classified as SNIa. If the data were not well fit by the template with the lowest $\chi^{2}$ value, they were classified as nIa.

Of course, this method is far from foolproof and it is possible that some SNe have been misclassified as SNIa. For example, as a test we applied the above method to SN 1997cl, the only spectroscopically classified SNII-n (a type of SN whose light curves could look like those of a SNIa) in our sample. The template with the lowest chi-squared value does indeed fit the data nicely, and under our scheme, this SN would have been misclassified as a SNIa. This demonstrates once again the importance of obtaining maximum-light spectra in order to unequivocally classify each SN. However, we do not have this information, and proceed assuming that all of the SNe we classified as SNIa, are indeed of this type. 
Table 3. The $\Delta m_{15}, B_{\max }, V_{\max }, R_{\max }, I_{\max }, z$ and the distance modulus for each of the SNe determined to be SNIa. Also included are the values we derived for the SNe listed in Phillips et al. (1999). The errors are listed in parentheses in units of $0.01^{\mathrm{m}}$.

\begin{tabular}{|c|c|c|c|c|c|c|c|}
\hline $\mathrm{SN}$ & $\Delta m_{15}$ & $B_{\max }$ & $V_{\max }$ & $R_{\max }$ & $I_{\max }$ & $z$ & $\begin{array}{c}\Delta m_{15} \\
\text { distance }\end{array}$ \\
\hline SN 1986G & $1.58(21)$ & $12.08(02)$ & $11.16(03)$ & - & - & 0.0018 & $27.40(10)$ \\
\hline SN 1989B & $1.27(11)$ & $12.25(03)$ & $11.80(03)$ & $11.61(03)$ & $11.60(04)$ & 0.0024 & $29.73(04)$ \\
\hline SN 1990N & $0.94(21)$ & $12.67(02)$ & $12.70(02)$ & $12.67(01)$ & $12.91(02)$ & 0.0034 & $32.18(03)$ \\
\hline SN 19900 & $0.93(10)$ & $16.15(04)$ & $16.16(05)$ & $16.16(06)$ & $16.53(07)$ & 0.0303 & $35.72(04)$ \\
\hline SN 1990T & $1.09(10)$ & $17.19(05)$ & $17.10(05)$ & $17.09(03)$ & $17.32(03)$ & 0.0404 & $36.22(08)$ \\
\hline SN 1990Y & $0.90(11)$ & $17.65(10)$ & $17.32(08)$ & $17.21(08)$ & $17.54(09)$ & 0.0391 & $35.85(07)$ \\
\hline SN 1990af & $1.58(21)$ & $17.76(03)$ & $17.74(02)$ & - & - & 0.0506 & $36.54(04)$ \\
\hline SN 1991S & $0.90(11)$ & $17.78(14)$ & $17.77(11)$ & $17.73(11)$ & $17.99(10)$ & 0.0546 & $37.18(12)$ \\
\hline SN 1991T & $1.00(10)$ & $11.62(01)$ & $11.46(01)$ & $11.42(01)$ & $11.62(01)$ & 0.0058 & $30.44(06)$ \\
\hline SN 1991U & $0.95(11)$ & $16.52(13)$ & $16.41(10)$ & $16.39(08)$ & $16.56(07)$ & 0.0317 & $35.51(09)$ \\
\hline SN 1991ag & $0.89(20)$ & $14.30(03)$ & $14.29(02)$ & $14.31(04)$ & $14.66(05)$ & 0.0141 & $33.75(08)$ \\
\hline SN 1992A & $1.43(10)$ & $12.52(01)$ & $12.53(01)$ & $12.51(01)$ & $12.81(01)$ & 0.0063 & $31.59(05)$ \\
\hline SN 1992J & $1.46(13)$ & $17.73(08)$ & $17.54(05)$ & - & $17.86(06)$ & 0.0446 & $36.34(10)$ \\
\hline SN 1992K & $1.88(20)$ & $15.86(03)$ & $15.17(04)$ & - & $14.92(05)$ & 0.0103 & $32.47(08)$ \\
\hline SN 1992P & $0.89(10)$ & $16.04(04)$ & $16.09(02)$ & - & $16.39(05)$ & 0.0252 & $35.59(07)$ \\
\hline SN 1992ae & $1.20(11)$ & $18.43(07)$ & $18.47(05)$ & - & - & 0.0752 & $37.69(21)$ \\
\hline SN 1992ag & $1.13(12)$ & $16.35(06)$ & $16.07(04)$ & - & $16.42(07)$ & 0.0249 & $34.96(09)$ \\
\hline SN 1992al & $1.08(10)$ & $14.49(02)$ & $14.52(02)$ & $14.59(02)$ & $14.86(02)$ & 0.0146 & $33.97(04)$ \\
\hline SN 1992aq & $1.46(13)$ & $19.27(08)$ & $19.31(05)$ & - & $19.78(08)$ & 0.1018 & $38.45(15)$ \\
\hline SN 1992au & $1.40(15)$ & $18.14(12)$ & $18.08(07)$ & - & $18.58(06)$ & 0.0614 & $37.32(12)$ \\
\hline SN 1992bc & $0.85(20)$ & $15.08(01)$ & $15.17(01)$ & $15.19(01)$ & $15.59(01)$ & 0.0202 & $34.79(02)$ \\
\hline SN 1992bg & $1.08(10)$ & $16.69(05)$ & $16.72(04)$ & - & $17.01(05)$ & 0.0352 & $36.02(10)$ \\
\hline SN 1992bh & $1.02(10)$ & $17.60(05)$ & $17.54(04)$ & - & $17.79(04)$ & 0.0450 & $36.66(16)$ \\
\hline SN 1992bk & $1.46(22)$ & 18.17(09) & $18.18(06)$ & - & $18.43(06)$ & 0.0581 & $37.20(10)$ \\
\hline SN 1992bl & $1.43(10)$ & $17.30(04)$ & $17.36(03)$ & - & $17.70(04)$ & 0.0437 & $36.46(05)$ \\
\hline SN 1992bo & $1.72(20)$ & $15.76(02)$ & $15.76(02)$ & $15.70(02)$ & $16.00(02)$ & 0.0189 & $34.56(02)$ \\
\hline SN 1992bp & $1.33(10)$ & $18.27(04)$ & $18.35(03)$ & - & $18.74(04)$ & 0.0793 & $37.55(05)$ \\
\hline SN 1992br & $1.88(20)$ & $19.59(11)$ & $19.37(06)$ & - & - & 0.0882 & $38.09(08)$ \\
\hline SN 1992bs & $1.08(10)$ & $18.26(05)$ & $18.26(04)$ & - & - & 0.0637 & $37.55(16)$ \\
\hline SN 1993B & $1.06(13)$ & $18.39(07)$ & $18.35(06)$ & - & $18.62(06)$ & 0.0696 & $37.44(08)$ \\
\hline SN $1993 \mathrm{H}$ & $1.72(20)$ & $16.75(03)$ & $16.49(02)$ & $16.43(02)$ & $16.58(03)$ & 0.0239 & $34.96(04)$ \\
\hline SN 19930 & $1.20(11)$ & $17.60(04)$ & $17.66(02)$ & - & $18.03(03)$ & 0.0510 & $36.99(06)$ \\
\hline SN 1993ac & $1.06(13)$ & 17.77(09) & $17.77(09)$ & $17.59(08)$ & 17.81(09) & 0.0493 & $36.76(15)$ \\
\hline SN 1993ae & $1.43(10)$ & $15.32(03)$ & $15.37(03)$ & $15.40(03)$ & $15.63(02)$ & 0.0190 & $34.47(03)$ \\
\hline SN 1993ag & $1.20(10)$ & $17.79(03)$ & $17.65(02)$ & - & $17.86(03)$ & 0.0490 & $36.89(11)$ \\
\hline SN 1993ah & $1.20(10)$ & $16.37(08)$ & $16.32(05)$ & - & $16.69(04)$ & 0.0297 & $35.57(09)$ \\
\hline SN 1994D & $1.20(10)$ & $11.85(01)$ & $11.84(01)$ & $11.85(01)$ & $12.17(01)$ & 0.0015 & $31.10(07)$ \\
\hline SN 1994M & $1.32(11)$ & $16.33(03)$ & $16.21(03)$ & $16.14(03)$ & $16.33(03)$ & 0.0230 & $34.99(06)$ \\
\hline
\end{tabular}

The photometric parameters from the fits to the Phillips et al. (1999) SNe and the SNe from the Mount Stromlo Abell cluster SN search deemed to be SNIa, are shown in Table 3. The multi-colour fits for the SNIa from the Mount Stromlo Abell cluster SN search can be found at the EDP Sciences website (http: //WwW . edpsciences . org/).

\subsubsection{Establishing a reddening-free $\Delta m_{15}$ relation}

To measure distances via Eq. (9), we need to establish the absolute magnitude of a SNIa for a set of filters $\left(B, V, R_{c}, I_{c}\right)$, based on $\Delta m_{15}$ and $M_{\Delta m_{15}}$. To do this, we require a set of SNIa, with a large range of $\Delta m_{15}$, known relative distances, and known reddenings. Phillips et al. (1999) has measured host galaxy extinction based on their late time light curves, colour evolution, and host type for a large number of objects. We use a subsample of their objects with $c z>3000 \mathrm{~km}^{-1} \mathrm{~s}^{-1}$ and with a measured host galaxy reddening $\left(E(B-V)_{\text {Avg }}\right)$ of less than 0.06 . These $\mathrm{SNe}$ are assumed to be unreddened by their host galaxy and to be at a high enough redshift that their peculiar velocities are relatively small. The distance to each SN was calculated from the redshift of the host galaxy, assuming $H_{0}=65 \mathrm{~km} \mathrm{~s}^{-1} \mathrm{Mpc}^{-1}$, and an additional peculiar velocity error of $400 \mathrm{~km} \mathrm{~s}^{-1}$ was included in the error bars of the absolute 
Table 3. continued.

\begin{tabular}{|c|c|c|c|c|c|c|c|}
\hline SN & $\Delta m_{15}$ & $B_{\max }$ & $V_{\max }$ & $R_{\max }$ & $I_{\max }$ & $z$ & $\begin{array}{c}\Delta m_{15} \\
\text { distance }\end{array}$ \\
\hline SN 1994Q & $0.90(11)$ & $16.36(12)$ & $16.32(10)$ & $16.23(10)$ & $16.61(09)$ & 0.0290 & $35.64(10)$ \\
\hline SN 1994S & $0.98(10)$ & $14.75(03)$ & $14.79(02)$ & $14.86(03)$ & $15.16(02)$ & 0.0151 & $34.36(03)$ \\
\hline SN 1994T & $1.46(22)$ & $17.20(14)$ & $17.15(09)$ & $17.00(08)$ & $17.39(10)$ & 0.0347 & $35.89(13)$ \\
\hline SN 1994ae & $0.93(10)$ & $13.05(01)$ & $13.01(01)$ & $12.94(01)$ & $13.31(02)$ & 0.0043 & $32.33(08)$ \\
\hline SN 1995D & $1.02(10)$ & $13.18(02)$ & $13.24(02)$ & $13.25(02)$ & $13.59(02)$ & 0.0065 & $32.73(07)$ \\
\hline SN 1995E & $1.11(10)$ & $16.68(03)$ & $15.96(03)$ & $15.50(03)$ & $15.33(03)$ & 0.0116 & $32.89(07)$ \\
\hline SN 1995ac & $1.00(10)$ & $17.10(02)$ & $17.15(02)$ & $17.14(02)$ & $17.24(03)$ & 0.0500 & $36.65(06)$ \\
\hline SN 1995ak & $1.33(10)$ & $15.92(05)$ & $15.93(04)$ & $15.77(03)$ & $15.97(04)$ & 0.0230 & $34.65(07)$ \\
\hline SN 1995al & $0.79(20)$ & $13.30(01)$ & $13.26(02)$ & $13.22(02)$ & $13.48(02)$ & 0.0051 & $32.68(14)$ \\
\hline SN 1995bd & $0.88(20)$ & $15.20(02)$ & $14.94(01)$ & $14.82(02)$ & $15.06(02)$ & 0.0160 & $33.98(10)$ \\
\hline SN 1996C & $0.89(20)$ & $16.47(04)$ & $16.41(04)$ & $16.35(05)$ & $16.66(05)$ & 0.0296 & $35.62(06)$ \\
\hline SN 1996X & $1.33(10)$ & $12.98(02)$ & $13.00(02)$ & $13.01(02)$ & $13.32(02)$ & 0.0068 & $32.19(07)$ \\
\hline SN $1996 Z$ & $1.11(15)$ & $14.27(12)$ & 13.91(09) & $13.82(11)$ & - & 0.0076 & $32.45(14)$ \\
\hline SN 1996ag † & $1.26(18)$ & $19.89(09)$ & $19.48(23)$ & $19.82(10)$ & $20.39(21)$ & 0.140 & $39.27(14)$ \\
\hline SN 1996ai & $0.79(20)$ & $16.86(02)$ & $15.25(02)$ & 14.44 & 14.00 & 0.0032 & $30.15(09)$ \\
\hline SN 1996aj & $1.26(18)$ & $19.77(17)$ & $19.42(22)$ & $19.07(12)$ & $19.77(25)$ & 0.110 & $37.66(43)$ \\
\hline SN 1996am & $0.90(11)$ & $18.54(05)$ & $18.28(04)$ & $18.38(04)$ & $18.61(08)$ & 0.0650 & $37.55(12)$ \\
\hline SN 1996ao & $0.90(11)$ & $18.62(21)$ & $18.26(14)$ & $18.16(14)$ & $17.80(21)$ & 0.0580 & $36.91(28)$ \\
\hline SN 1996bk & $1.88(20)$ & 15.29 & 14.5 & 14.22 & 14.34 & & $31.88(09)$ \\
\hline SN 1996bl & $1.08(10)$ & $16.65(03)$ & $16.60(02)$ & $16.62(02)$ & $16.87(03)$ & 0.0360 & $35.85(07)$ \\
\hline SN 1996bo & $1.30(10)$ & $15.83(02)$ & $15.51(02)$ & $15.24(02)$ & $15.53(02)$ & 0.0173 & $33.75(03)$ \\
\hline SN 1996bv & $0.90(20)$ & 15.27 & 15.10 & 14.96 & 15.12 & 67 & $33.77(06)$ \\
\hline SN 1996bx & $1.46(22)$ & $18.05(36)$ & $19.27(32)$ & $18.35(15)$ & $20.81(63)$ & 0.0580 & $37.04(62)$ \\
\hline SN 1997by & $1.43(10)$ & $17.28(05)$ & $17.26(03)$ & $17.29(03)$ & $17.39(06)$ & 0.0450 & $36.24(09)$ \\
\hline SN 1997bz & $1.04(22)$ & - & $18.73(07)$ & 18.11(07) & $18.19(49)$ & 0.0300 & $35.21(54)$ \\
\hline SN 1997cp $\dagger$ & $1.34(21)$ & $19.69(54)$ & - & $19.53(49)$ & - & & $39.69(45)$ \\
\hline SN 1997cu & $1.54(23)$ & - & $18.13(16)$ & $18.08(16)$ & - & 0.0620 & $37.07(14)$ \\
\hline SN $1997 \mathrm{dr} \dagger$ & $1.88(20)$ & $18.56(20)$ & $18.81(11)$ & $18.64(08)$ & $18.76(27)$ & 0.0750 & $37.35(09)$ \\
\hline SN 1997fb & 1.06 & & & & & & $36.92(14)$ \\
\hline SN 1997fc & $0.94(11)$ & $17.47(08)$ & $17.59(05)$ & $17.55(04)$ & $17.75(08)$ & 0.0540 & $36.99(08)$ \\
\hline SN $1997 \mathrm{fd} \dagger$ & $1.26(25)$ & $20.45(16)$ & - & $20.84(17)$ & - & 0.190 & $40.31(27)$ \\
\hline SN 1998Z & $1.06(22)$ & - & $17.65(16)$ & $17.51(14)$ & - & 0.0380 & $37.16(39)$ \\
\hline SN 1998br† & $1.26(18)$ & $18.87(08)$ & - & $18.70(07)$ & - & 0.0810 & $37.96(26)$ \\
\hline SN 1998bu & $1.08(10)$ & $12.10(01)$ & $11.79(01)$ & $11.63(01)$ & $11.66(01)$ & 0.0025 & $30.05(05)$ \\
\hline SN $1998 \mathrm{~cm}$ & $0.90(20)$ & $18.51(10)$ & - & $18.36(06)$ & - & 0.0800 & $37.75(24)$ \\
\hline SN $1998 \mathrm{do}^{\dagger} \dagger$ & $1.34(21)$ & $18.57(21)$ & - & $18.90(19)$ & - & 0.0920 & $38.18(30)$ \\
\hline SN 1998dv $\dagger$ & $1.34(21)$ & $19.71(32)$ & - & $19.67(28)$ & - & 0.155 & $39.27(59)$ \\
\hline SN 1998dw & $1.27(11)$ & - & $17.17(29)$ & $17.04(32)$ & - & 0.0490 & $36.91(18)$ \\
\hline SN 1998dz & $1.01(10)$ & $18.79(07)$ & $18.90(12)$ & $18.82(06)$ & $19.06(18)$ & 0.0910 & $38.11(14)$ \\
\hline SN 1998ea & $1.06(13)$ & $19.87(55)$ & $17.82(07)$ & $18.15(07)$ & 19.71(80) & 0.0570 & $37.01(17)$ \\
\hline $\mathrm{SN} 1998 \mathrm{fb} \dagger$ & $1.34(27)$ & - & $17.61(38)$ & $17.99(21)$ & - & 0.0597 & $37.18(23)$ \\
\hline SN 1999ao & $0.90(11)$ & $17.78(05)$ & $17.83(05)$ & $17.77(04)$ & $18.15(06)$ & 0.0550 & $37.00(08)$ \\
\hline
\end{tabular}

magnitudes (Marzke et al. 1995). From this subsample, we derive the reddening-free luminosity-decline rate relations in $B$, $V, R$ and $I$ shown in Fig. 2.

Given the large errors in $\Delta m_{15}$, the least squares fits to the unreddened luminosity-decline rate relations were initially assumed to be linear. However, we found that those SNIa with extreme values of $\Delta m_{15}$ did not fit the Hubble line. Phillips et al. (1999) found that the peak luminosity-decline rate relations were nonlinear in the $B$ and $V$ bands and possibly in the $I$ band. By recalculating the distance moduli with quadratic least squares fits to the $B$ and $V$ data, the dispersion in the Hubble diagram was reduced significantly (i.e. more than compensated for the extra degree of freedom). This was not the case for the $R$ and $I$ band relations, and these were kept as linear fits. 


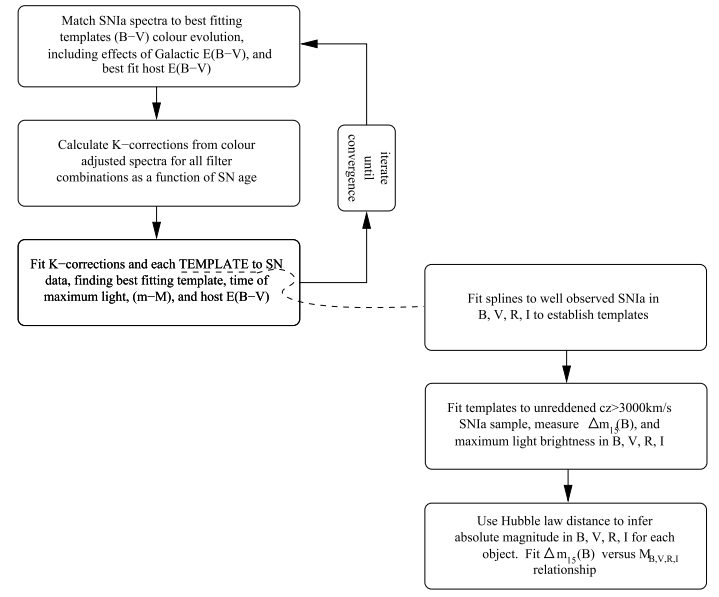

Fig. 2. The luminosity-decline rate relations in $B, V, R$ and $I$ for those $\mathrm{SNe}$ from the sample of Phillips et al. (1999) with host galaxy reddenings measured to be less than 0.06 . The solid lines in $B$ and $V$ indicate the quadratic fits to the data, the dashed lines are the linear fits for reference.

The unreddened luminosity-decline rate relations are shown in Eqs. (11)-(14).

$$
\begin{aligned}
B_{\max }= & -19.47( \pm 0.06)+0.74( \pm 0.33)\left(\Delta m_{15}-1.1\right)+ \\
& 0.70( \pm 0.56)\left(\Delta m_{15}-1.1\right)^{2}
\end{aligned}
$$

$$
V_{\max }=-19.42( \pm 0.06)+0.75( \pm 0.32)\left(\Delta m_{15}-1.1\right)+
$$$$
0.25( \pm 0.53)\left(\Delta m_{15}-1.1\right)^{2}
$$

$R_{\max }=-19.42( \pm 0.12)+0.84( \pm 0.31)\left(\Delta m_{15}-1.1\right)$

$I_{\max }=-19.06( \pm 0.08)+0.83( \pm 0.27)\left(\Delta m_{15}-1.1\right)$.

\subsubsection{The pros and cons of $\Delta m_{15}$}

One of the major problems with the $\Delta m_{15}$ method lies in determining the time of $B$-maximum (Sect. 4.4.1). A very small change in $t_{0}^{B}$ can change the $\Delta m_{15}$ measured for the SN by quite a large amount. This is especially problematic for $\mathrm{SNe}$ like SN 1991T that are very flat at maximum and where, even with very good data, the $t_{0}^{B}$ is difficult to constrain.

Perhaps it is worthwhile to revisit the original $\beta$ parameter introduced by Pskovskii $(1967,1977)$ which measures the decline rate directly as the $B$ band post-maximum decline in magnitudes per 100 days. This parameter is unaffected by the exact time of $B$-maximum, and could be determined from the peak in the derivative of a fit to the SN data. With the well-sampled, CCD-based SNIa light curves now being collected around the world, this should be a relatively straightforward procedure and more revealing than when first attempted by Pskovskii.

The real strength of the $\Delta m_{15}$ method is that it fits the $\mathrm{SN}$ data to templates created directly from well-observed SN light curves. The Stretch (Perlmutter et al. 1997) and Multi-colour Light Curve Shapes (MLCS; Riess et al. 1995, 1996) techniques on the other hand, take these original templates and create "intermediate" templates which capture most of the nuances of SN light curves, but not all. Although not perfect, these

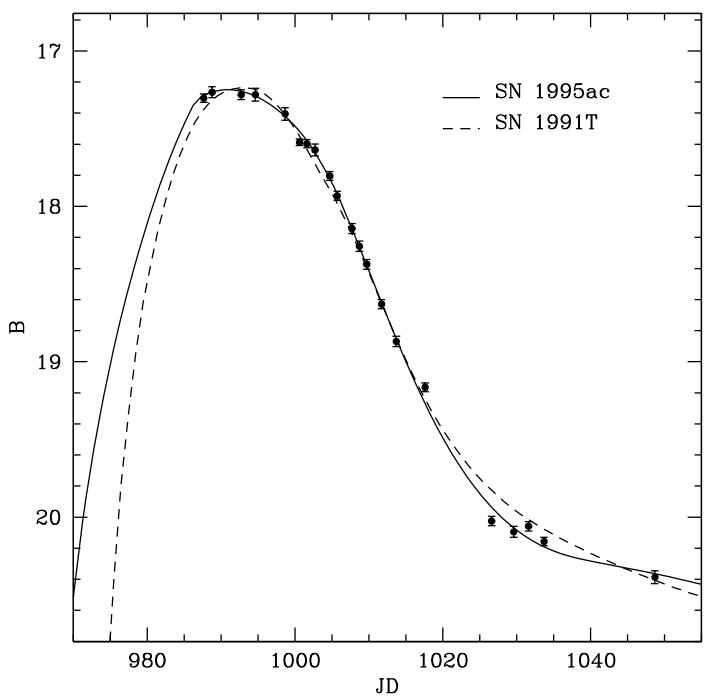

Fig. 3. Illustration, using template fits to the data of SN 1995ac, of one of the problems with the $\Delta m_{15}$ method which arises due to the discreteness of the $\Delta m_{15}$ values of the template light curves. The solid line is a template created from the data of SN 1995ac and the dashed line is the template of SN 1991T, which is the best-fit template if the SN 1995 ac template is excluded in the $\Delta m_{15}$ fits.

"intermediate" templates overcome the second major problem with the $\Delta m_{15}$ method - it relies on a finite number of templates with discrete values of $\Delta m_{15}$. With only 15 templates spanning the entire range of SN decline rates, the steps between the values of $\Delta m_{15}$ for the templates fitted to the data can result in the wrong value of $\Delta m_{15}$ for the SN, particularly for those SNe with $\Delta m_{15}>1.45$ and $\Delta m_{15}<0.9$. A good example of this limitation is SN 1995ac. A template was constructed for this $\mathrm{SN}$ as in Sect. 4.4.1 and was found to have a $\Delta m_{15}$ of 0.686. If, however, the SN 1995ac template is removed and only the remaining 15 templates fit to the data, the best fit template is that of SN $1991 \mathrm{~T}$ with a $\Delta m_{15}$ of 1.0. The differences between these fits is shown in Fig. 3. It is immediately apparent that the SN 1995ac template fits the data much better than the SN 1991T template (as one would expect since it is created from the data itself) and hence the measured $\Delta m_{15}$ derived from the fitting process is too large.

The discreteness of the method will, of course, become less of a problem as a larger sample of well observed SNe with data starting before $B$ maximum is collected. Indeed, Hamuy et al. (1995) attempted to correct for this by fitting a parabola to the reduced $\chi^{2}$ values resulting from their template fits. But for the present time, it poses a real problem for our implementation of the $\Delta m_{15}$ technique.

That being said - the tight fit of the data around the Hubble line (see Sect. 5.1) and the agreement in the distances to two of the Mount Stromlo Abell cluster SN search SNe which occurred in the same cluster, SN $1997 \mathrm{fb}$ and SN $1997 \mathrm{fc}$, illustrate that the $\Delta m_{15}$ method does work remarkably well, even for our sparsely sampled dataset. While not found in the same galaxy, SN $1997 \mathrm{fb}$ and SN $1997 \mathrm{fc}$ both occurred in the same cluster and we expect their distance moduli to be very similar. As can be seen from Table 3 this is indeed the case. Although they had different decline rates, hence different values for $\Delta m_{15}$, and 


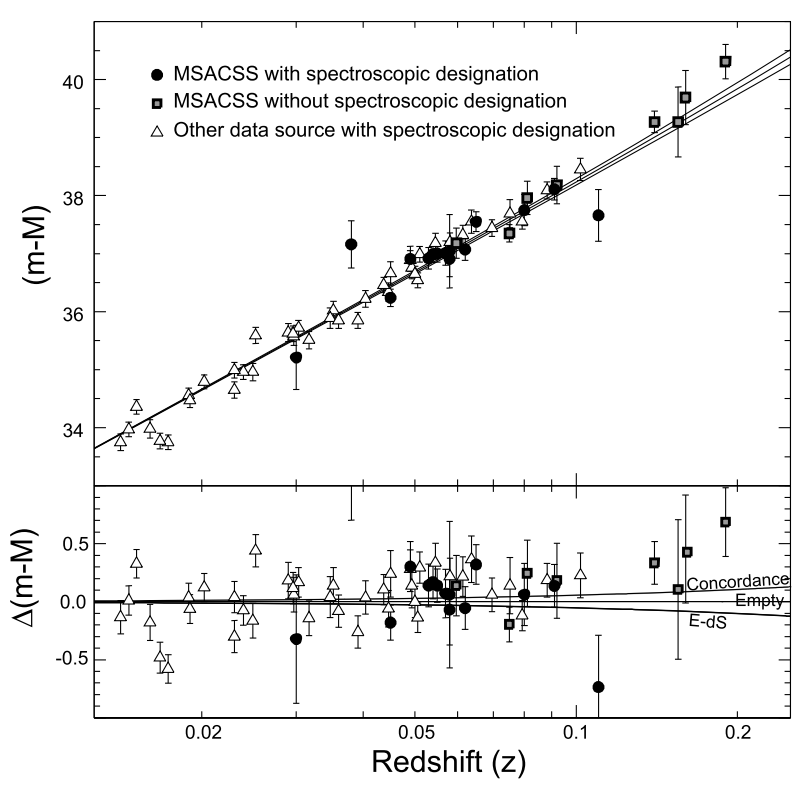

Fig. 4. Top panel: the Hubble diagram constructed with the SNe from the Mount Stromlo Abell cluster SN search, and the Phillips et al. (1999) SNIa with $z>0.01$. Bottom panel: the distances shown as a residual Hubble diagram, relative to an Empty Universe. Lines showing the luminosity distances for a Concordance Cosmology $\left(\Omega_{\Lambda}=\right.$ $0.72, \Omega_{\mathrm{M}}=0.28$ ), empty Universe, and Einstein de-Sitter Universe are are plotted).

were fit by different templates (1.06/SN 1995E for SN $1997 \mathrm{fb}$ and $0.94 / \mathrm{SN} 1991 \mathrm{~T}$ for SN 1997fc), the data are fit quite well by the corresponding templates and the same distance is measured to within the uncertainties.

\section{Discussion}

All of the above has described our data reduction techniques, how we constructed our SN light curves and our implementation of the $\Delta m_{15}$ method. This is important to describe in detail so that other researchers may develop software similar to ours. However, what have we learned from all this data reduction?

\subsection{The Hubble diagram}

We can use the SNIa with $z>0.01$ from Phillips et al. (1999) and our own search to accurately probe the Hubble flow. Figure 4 shows the Hubble diagram for this sample of SNIa, corrected for Galactic reddening, host galaxy reddening and the luminosity-decline rate relation. Our $\Delta m_{15}$ distances are intrinsically relative to each other. We place them on an absolute scale by finding the offset which matches them to the cosmological line of $\Omega_{\mathrm{M}}=0.3, \Omega_{\Lambda}=0.7$ with $H_{0}=$ $70 \mathrm{~km}^{-1} \mathrm{~s}^{-1} \mathrm{Mpc}^{-1}$.

The two outliers in the Hubble diagram, SN 1996aj and SN 1998Z, are spectroscopically confirmed SNIa from the Mount Stromlo Abell cluster SN search which contain little photometric information to tie down the template fit. With so few points, it is difficult to constrain the light curve width and hence the distance to the $\mathrm{SN}$. It is therefore not a surprise that the calibration fails. The lack of other outliers suggests that the ranking of SNe based on the goodness of fit of the template light curves has probably done a reasonable job of selecting the SNIa from the untyped SNe. However, there is no way of knowing whether some true SNIa have been eliminated in this process, or whether some of the SNe included in the SNIa sample are of some other type. For example, the previously mentioned SNII-n, SN 1997cl, when plotted on this Hubble diagram, also lies on the Hubble line.

Based on the criteria described above, the sample of SNe listed in Table 3 includes all photometrically and spectroscopically classified SNIa. The objects with photometric classification are indicated with $\mathrm{a} \dagger$ to reflect their uncertain designation. Figure 4 shows a number of features which are worth discussing.

The dispersion about the Hubble line, discarding the two outliers, is $\sigma=0.19(\sigma=0.17$ over the same range as Phillips et al. (1999) This is larger than the $\sigma=0.14$ that Phillips et al. (1999) derive, probably due to: 1) the Mount Stromlo Abell cluster SN search data included here is much poorer than the data used by Phillips; and 2) SNe with $B_{\max }-V_{\max }>0.2$ are included. Since the error estimates in Table 3 only include statistical noise, and do not include the intrinsic dispersion of the SNIa class, we find that we must add $0.12 \mathrm{mag}$ in quadrature to each of our $\Delta m_{15}$ distances in the table to explain the scatter about the Hubble line above $z>0.03$ where peculiar motions are not significant.

Zehavi et al. (1998) claimed to see a local Hubble bubble, where the Hubble law changed value, at approximately $z=0.06$. This change in $H_{0}$ is noticeable in Fig. 4 , with the data sample presented here providing distances consistent with those used by Zehavi at $z>0.06$. However, since our dataset does not probe $z<0.05$ and therefore misses the putative transition region, we leave it to future datasets to examine this feature more closely.

To measure $H_{0}$, it is necessary to tie our $\Delta m_{15}$ distances to objects which have Cepheid distances to their host galaxies. We only include objects that were observed with modern electronic detectors. The Cepheid distances and our derived SN distances for each object (from Sect. 4.4.3) are listed in Table 4. For each SN host galaxy, there are two Cepheid distances listed. These were collated by Jha (2002; thesis Harvard University) - one using the methodology summarised by Saha et al. (2001a), the other using the methodology summarised in Freedman et al. (2001). Using the MLCS method, Jha (2002) has argued that systematic differences in the distances from the two Cepheid distance methodologies exceeds the statistical noise in the comparison of SN and Cepheid distances. Table 4 shows a similar pattern using our $\Delta m_{15}$ distances.

For each Cepheid distance methodology, we derive a value of $H_{0}$ based on a $\chi^{2}$ minimisation (equivalent to a weighted average) to match the Cepheid and SN distances, where the Cepheids provide the absolute calibration of the SNIa distance, and the SNIa provide the probe of the Hubble flow itself. The relative SN distances pin down the Hubble flow precisely, with the $1 \sigma$ uncertainty (not including Cepheid calibration) on $H_{0}$ being $1.5 \%$. Excluding or including the photometrically classified SNIas changes the derived $H_{0}$ values by less than $0.2 \%$. 
Table 4. Cepheid Calibration of SNIa for Measuring $H_{0}$.

\begin{tabular}{ccccc}
\hline \hline SN & Host & $\mu$ (Saha et al. 2001a) & $\mu($ Freedman et al. 2001) & $\mu\left(\Delta m_{15} H_{0}=70\right)$ \\
$1972 \mathrm{E}$ & NGC 5253 & $27.93 \pm 0.08$ & $27.49 \pm 0.15$ & $27.85 \pm 0.14$ \\
$1981 \mathrm{~B}$ & NGC 4536 & $31.17 \pm 0.13$ & $30.87 \pm 0.06$ & $31.14 \pm 0.14$ \\
$1989 \mathrm{~B}$ & NGC 3627 & $30.37 \pm 0.13$ & $30.01 \pm 0.06$ & $29.73 \pm 0.13$ \\
1990N & NGC 4639 & $32.13 \pm 0.22$ & $31.71 \pm 0.09$ & $32.18 \pm 0.12$ \\
$1991 \mathrm{~T}$ & NGC 4527 & $30.82 \pm 0.13^{a}$ & $30.61 \pm 0.09^{b}$ & $30.44 \pm 0.13$ \\
$1998 \mathrm{bu}$ & NGC 3368 & $30.51 \pm 0.16$ & $30.11 \pm 0.07$ & $30.05 \pm 0.13$ \\
\hline
\end{tabular}

${ }^{a}$ From Saha et al. (2001bb).

${ }^{b}$ From Gibson \& Stetson (2001).

We find using the Saha et al. (2001a) Cepheid distances, $H_{0}=62 \pm 3 \mathrm{~km}^{-1} \mathrm{~s}^{-1} \mathrm{Mpc}^{-1}$, with $\chi^{2}=9.9$ for 5 degrees of freedom, whereas the Freedman et al. (2001) distances give $H_{0}=73 \pm 3 \mathrm{~km}^{-1} \mathrm{~s}^{-1} \mathrm{Mpc}^{-1}$, with $\chi^{2}=16.9$ for 5 degrees of freedom. The larger than expected $\chi^{2}$ values can be explained by underestimated errors, either on the part of the Cepheids, or the SNe. These values are consistent with the values derived by Saha et al. (2001a), $H_{0}=61 \pm 2 \mathrm{~km}^{-1} \mathrm{~s}^{-1} \mathrm{Mpc}^{-1}$, and Freedman et al. (2001), $H_{0}=71 \pm 2 \mathrm{~km}^{-1} \mathrm{~s}^{-1} \mathrm{Mpc}^{-1}$, using slightly different subsets of these SN with their own SN distance methodology. In addition, all of the above quoted values of $H_{0}$ have in common a systematic uncertainty component of $\pm 10 \%$ related to the distance to the LMC (assumed to be $(m-M)=18.5)$ as well as HST calibration uncertainties. The large difference between the value of $H_{0}$ derived using distances from the two Cepheid methods (and the statistical consistency within either set of Cepheids) argues strongly that the largest uncertainty in $H_{0}$ has nothing to do with SN, but rather the use of Cepheids as calibrators. Taking the discrepancy between the two Cepheid distance methodologies at face value, and including the systematic error common to both teams, we find, using our $\Delta m_{15}$ distances, a value of $53<H_{0}<$ $83 \mathrm{~km}^{-1} \mathrm{~s}^{-1} \mathrm{Mpc}^{-1}$, where this range is largely dictated by systematic error, not statistical error. This uncertainty is much larger than is normally quoted for the Hubble constant, but our analysis indicates this is the true range of the current uncertainty, in concordance with the work of Jha (2002).

\subsection{Acceleration of the Universe}

SNIa have gained special notoriety because of the inferred acceleration of the Universe based on distances to several objects at $z>0.3$. One criticism of this work is the discontinuous redshift distribution of the sample, with a fraction of objects at $z<0.1$ and another fraction at $z>0.3$, but no connection in between. Since the acceleration is deduced by the relative faintness of objects at $z \sim 0.5$ compared to those at $z \sim 0.05$, it is desirable to test if the continuous low redshift sample by itself provides evidence for acceleration.

The deceleration parameter is a useful parameterisation of cosmological models for any combinations of matter, with sufficient accuracy at $z<0.2$. As a single parameter which measures acceleration, it is well suited to this test. Since $q_{0}=\frac{1}{2} \sum_{i} \Omega_{i}\left(1+3 w_{i}\right)$, the "Concordance Cosmology", $\left(\Omega_{M}=\right.$ $0.3, \Omega_{\Lambda}=0.7$ ) has a large deceleration parameter, $q_{0}=-0.55$.

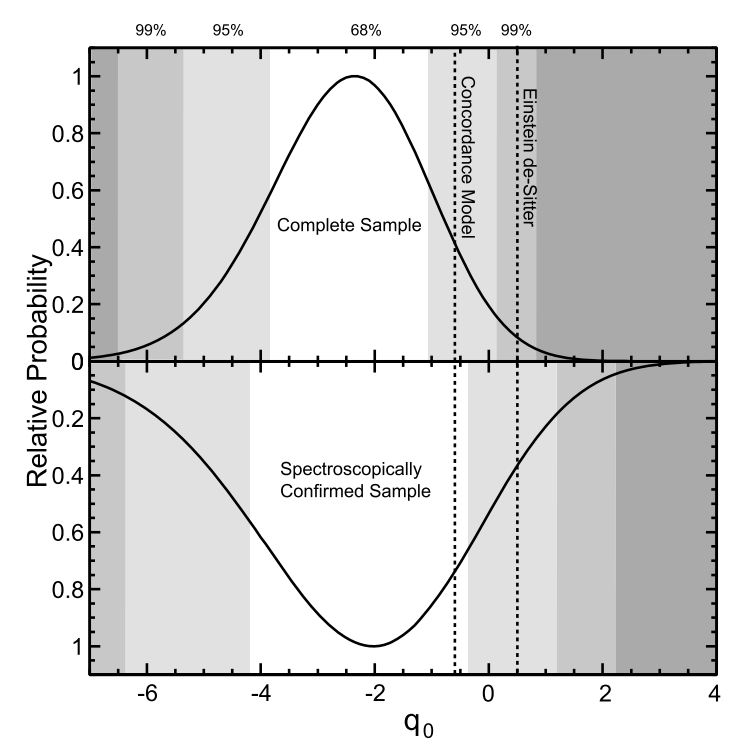

Fig. 5. Top panel: the relative probability of $q_{0}$ fit to all $\mathrm{SN}$ with $c z>3000 \mathrm{~km}^{-1} \mathrm{~s}^{-1}$. Bottom panel: same as top panel except objects without spectroscopic confirmation are excluded.

The Abell cluster SN search provides a significant increase in the number of objects at $0.05<z<0.2$, and therefore provides enough statistical leverage for a rough test.

We used all data from Table 3 with $z>0.03$, assumed a cosmic scatter of $0.12 \mathrm{mag}$ (added in quadrature to all error estimates in Table 3), marginalised over $H_{0}$, calculated $\chi^{2}$ for each value of $q_{0}$, and converted this to a probability via $p \propto \mathrm{e}^{\chi^{2}-\chi_{\min }^{2} / 2}$. Figure 5 shows that the $95 \%$ confidence region includes $-5.4<q_{0}<0.05$, with a Concordance Cosmology model ruled out at $84 \%$ (which is not highly significant), and models as extreme as Einstein-de Sitter $\left(\Omega_{\mathrm{M}}=1\right)$ Universe ruled out at $98 \%$ level.

The strength of these conclusions is largely based on the most distant objects in Table 3, and as illustrated in Fig. 4, a disproportionate number of these objects are without spectral classification. Richardson et al. (2002) have investigated luminosity distributions of various $\mathrm{SN}$ classes, and a non-negligible fraction of SN II-L, SN II-n, and SN Ib/c are bright enough and similar enough in light curve shape to be confused by our photometric classification scheme. However, these objects are not common. For example, from the Calàn/Tololo sample (Hamuy et al. 1996b), of the 50 objects discovered, 32 were SNIa, and 
2 were objects that could have been mistaken by our photometric classification process. Unfortunately, this mistaken classification rate of $(5 \%-10 \%)$ suggests it is possible that one, or even two of our photometrically classified objects are not SNIa. To account for this problem we apply a jack knife test where we remove each photometrically classified SN in turn, and rerun the analysis. The procedure reveals that while the exclusion of any one or two objects does not significantly change the best derived value for $q_{0}$, the tightness of the preferred region is very sensitive to the inclusion of both SN 1997fd $(z=0.19)$ and SN 1996ag $(z=0.14)$. If either of these objects is excluded, the $95 \%$ confidence region extends to $-5.12<q_{0}<0.69$ and $-5.63<q_{0}<0.54$, respectively. If we use only the spectroscopically confirmed objects, the $95 \%$ confidence limits are then $-6.46<q_{0}<1.20$.

We can also ask the question, is the Universe decelerating (i.e. is $q_{0}>0$ ) by integrating the total probability above $q_{0}=0$. Using the entire dataset from Table 3 , this calculation indicates there is a $97 \%$ chance the Universe is not decelerating. Excluding $1996 \mathrm{ag}$ and $1997 \mathrm{fd}$ changes the probability to $94 \%$ and $92 \%$, respectively. Including only spectroscopically confirmed objects, lowers the constraint to an $89 \%$ chance the Universe is not decelerating.

Clearly, the $z<0.2$ SNIa delineated in this paper are not only consistent with an accelerating Universe, but argue for it. However, much of the apparent strength of the argument comes from the fact that the best fit value for $q_{0}$ happens to lie on the negative side of the expected value, thereby making models with positive values of $q_{0}$ unfavourable. This is consistent with statistical fluctuations ( 1 in 6 datasets would differ from the Concordance model by this amount), but could also be due to any number of systematic effects, such as those discussed in Schmidt et al. (1998), or Perlmutter et al. (1999) because the effect being probed is so small. Of special concern are $\mathrm{K}$-corrections. Our observations of objects at $0.1<z<0.15$ do not match well to the rest frame (BVRI) bandpasses upon which our SN distance estimates rely. Selection effects should also be present, but will cause us to select preferentially bright objects and therefore underestimate distances. Selection effects therefore drive our experiment to overestimate $q_{0}$. In summary, this data set demonstrates that there is consistency of the low- $z$ SNIa with Concordance Cosmology model, but without significant augmentation to the sample it is impossible to make definitive statements.

\section{Conclusion}

Although not a popular target with currently active SN searches, the Mount Stromlo Abell cluster SN search has shown that finding and following $\mathrm{SNe}$ in the little observed redshift range of $0.02<z<0.2$ can be successfully achieved with a wide field camera mounted on a $1 \mathrm{~m}$ or $2 \mathrm{~m}$ class telescope. Taking into account the poor observing conditions in Australia, the discovery of $48 \mathrm{SNe}$ over the course of 3.5 years demonstrates that targeting rich galaxy clusters and careful scheduling of observations can yield many young SNe suitable for distance work at these important redshifts. However, the lack of spectroscopic classifications of the $\mathrm{SNe}$ in this search also highlight one of the problems of observing $\mathrm{SNe}$ at these redshifts: they are too faint to obtain good spectra with a $2 \mathrm{~m}$-class telescope in a short period of time.

Given the generally poor quality of the data obtained for these $\mathrm{SNe}$, we have once again reaffirmed the power of template-fitting techniques for deriving distances to SNIa, in this case using a modified $\Delta m_{15}$ technique. Although several problems were encountered with our implementation of this technique, the dispersion in the Hubble diagram constructed from the Mount Stromlo Abell cluster SN search SNIa and the SNIa presented in Phillips et al. (1999) was small $(\sigma=0.17)$ and our $\mathrm{SNe}$ are consistent with an accelerating universe.

This research was partially supported by NSF grant AST 9320045 and by grants from the Seaver Institute and the Packard Foundation to the University of Washington. Further support was provided by the Space Telescope Science Institute through grant GO-07505.02-96A and the Australian Research Council via grant s3504089. We would also like to thank the observatory director at MSSSO for his selection of this project for director's discretionary time and Lisa Germany thanks the director of CTIO for director's discretionary time on the CTIO $0.9 \mathrm{~m}$ and $1.5 \mathrm{~m}$ telescopes during her 8 month visit there. We appreciate the efforts of our referee who substantially improved this paper.

\section{References}

Abell, G. O., Corwin, H. G., Jr., \& Olowin, R. P. 1989, ApJS, 70, 1

Alard, C. 2000, A\&AS, 144, 363

Bertin, E., \& Arnouts, S. 1996, A\&A, 117, 393

Bessell, M. S. 1990, PASP, 102, 1181

Bessell, M. S. 1999, PASP, 111, 1426

Bessell, M. S. 1999, PASP, 111, 1421

Filippenko, A. V., Richmond, M. W., Branch, D., et al. 1992, AJ, 104, 1543

Filippenko, A. V. 1997, ARA\&A, 35, 309

Freedman, W. L., Madore, B. F., Gibson, B. K., et al. 2001, ApJ, 553, 47

Gal-Yam, A., et al. 2003, AJ, in preparation

Germany, L. M., Reiss, D. J., Schmidt, B. P., \& Stubbs, C. W. 2000, ApJ, 533, 320

Germany, L. M., \& Strolger, L.-G. 2003, in preparation

Gibson, B. K., Stetson, P. B., Freedman, W. L., et al. 2000, ApJ, 529, 723

Gibson, B. K., \& Stetson, P. B. 2001, ApJ, 547, L103

Groth, E. J. 1986, AJ, 91, 1244

Hamuy, M., Phillips, M. M., Wells, L. A., et al. 1993a, PASP, 105, 787

Hamuy, M., Maza, J., Phillips, M. M., et al. 1993b, AJ, 106, 2392

Hamuy, M., Phillips, M. M., Maza, J., et al. 1995, AJ, 109, 1

Hamuy, M., Phillips, M. M., Suntzeff, N. B., et al. 1996a, AJ, 112, 2438

Hamuy, M., Phillips, M. M., Suntzeff, N. B., et al. 1996b, AJ, 112, 2408

Hamuy, M., Phillips, M. M., \& Suntzeff, N. B. 1996c, AJ, 112, 2391

Harris, W. H., Fitzgerald, M. P., \& Reed, B. C. 1981, PASP, 93, 507

Hamuy, M., \& Pinto, P. A. 1999, AJ, 117, 1185

Jha, S., Garnavich, P. M., Kirshner, R. P., et al. 1999, ApJS, 125, 74

Kim, A., Goobar, A., \& Perlmutter, S. 1996, PASP, 108, 190

Landolt, A. U. 1992, AJ, 104, 340

Leibundgut, B., Kirshner, R. P., Phillips, M. M., et al. 1993, AJ, 105, 301 
Lira, P., Hamuy, M., Wells, L. A., et al. 1998, AJ, 115, 234

Marzke, R. O., Geller, M. J., da Costa, L. N., \& Huchra, J. P. 1995, AJ, 110, 447

Maza, J., Wischnjewsky, M., \& Torres, C. 1981, PASP, 93, 239

Oke, J. B., \& Sandage, A. 1968, ApJ, 154, 21

Perlmutter, S., Gabi, S., Goldhaber, G., et al. 1997, ApJ, 483, 595

Perlmutter, S., Aldering, G., Goldhaber, G., et al. 1999, ApJ, 517, 565

Phillips, M. M. 1993, ApJ, 413, L105

Philips, M. M., Lira, P., Suntzeff, N. B., et al. 1999, AJ, 118, 1766

Pskovskii, Y. 1967, Soviet Astron., 11, 63

Pskovskii, Y. 1977, Soviet Astron., 21, 675

Nugent, P., Kim, A., \& Perlmutter, S. 2002, PASP, 114, 803

Reiss, D. J., Germany, L. M., Schmidt, B. P., \& Stubbs, C. W. 1998, AJ, 115, 26

Reiss, D. J., Germany, L. M., Schmidt, B. P., \& Stubbs, C. W. 2003a, in preparation

Reiss, D. J., Germany, L. M., Schmidt, B. P., \& Stubbs, C. W. 2003b, in preparation

Richardson, D., Branch, D., Casebeer, D., et al. 2002, AJ, 123, 745

Riess, A. G., Press, B., \& Kirshner, R. P. 1995, ApJ, 438, L17

Riess, A. G., Press, B., \& Kirshner, R. P. 1996, ApJ, 473, 88

Riess, A. G., Filippenko, A. V., Challis, P., et al. 1998, AJ, 116, 1009

Riess, A. G., Kirshner, R. P., Schmidt, B. P., et al. 1999a, AJ, 117, 707
Riess, A. G., Filippenko, A. V., Li, W., et al. 1999b, AJ, 118, 2675

Saha, A., Sandage, A., Labhardt, L., et al. 1995, ApJ, 438, 8

Saha, A., Sandage, A., Tammann, G. A., et al. 2001a, ApJ, 562, 314

Saha, A., Sandage, A., Thim, F., et al. 2001b, ApJ, 551, 973

Savage, B. D., \& Mathis, J. S. 1979, ARA\&A, 17, 73

Schechter, P. L., Mateo, M., \& Saha, A. 1993, PASP, 105, 1342

Schlegel, D., Finkbeiner, D., \& Davis, M. 1998, ApJ, 500, 525

Schmidt, B. P., Suntzeff, N. B., Phillips, M. M., et al. 1998, ApJ, 507, 46

Schwartz, M. 1997, IAU Circ \#6700

Shaw, R. L. 1979, A\&A, 76, 188

Stetson, P. B. 1987, PASP, 99, 191

Stetson, P. B. 1996, PASP, 108, 851

Stubbs, C. W., Akerlof, C., Bennett, D., et al. 1993, Proc. SPIE, 192, 1900

Suntzeff, N. B., Phillips, M. M., Covarrubias, R., et al. 1999, AJ, 117, 1175

Treffers, R. R., Peng, C. Y., Filippenko, A. V., \& Richmond, M. W. 1997, IAU Circ. \#6627

Turatto, M., Benetti, S., Cappellaro, E., et al. 1996, MNRAS, 283, 1

Wang, L., Hoeflich, P., \& Wheeler, J. C. 1997, ApJL, 483, L29

Zehavi, I., Riess, A. G., Kirshner, R. P., \& Dekel, A., 1998, ApJ, 503, 483 


\section{Online Material}


L. M. Germany et al.: Results Mount Stromlo Abell cluster SN search, Online Material p 2

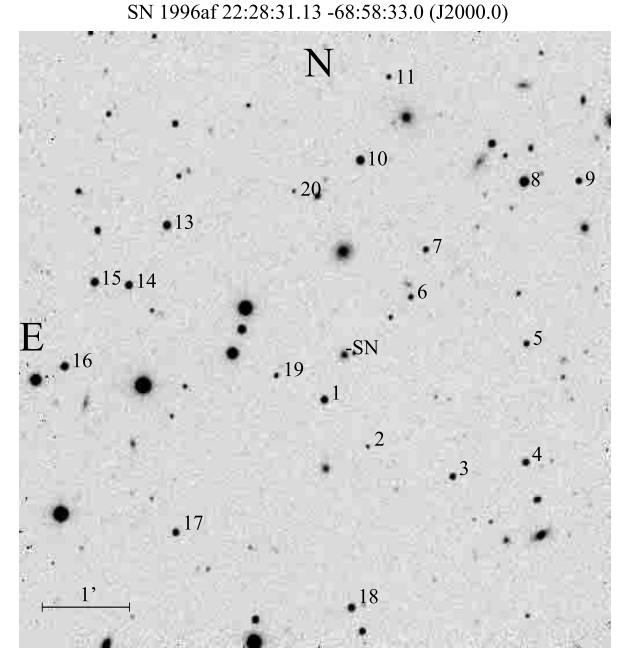

Fig. A.1. Finding chart for the local standard stars in the field of SN 1996af.

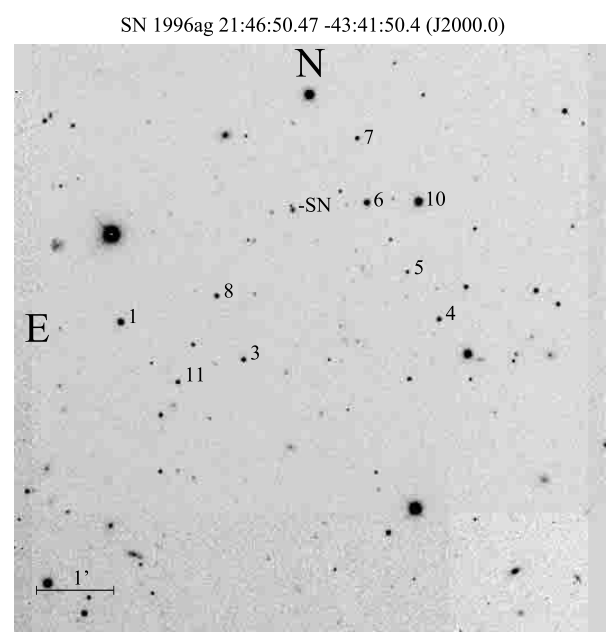

Fig. A.2. Finding chart for the local standard stars in the field of SN 1996ag.

\section{Appendix A: The Finding Charts}

In Sect. 4.2, the selection and calibration of the local standard stars was discussed. This Appendix presents the finding charts for each SN. The numbers in each field indicate the number of the local standard star whose $B V(R I)_{\mathrm{KC}}$ photometry is made available at the CDS (http://cdsweb.u-strasbg. fr/).

Each finding chart is a montage of all the $B$ images taken at CTIO during 1999 (Sect. 4.2) for that SN field. Since each SN was observed a different number of times on different telescopes, the exposure times for the finding charts vary from approximately $600 \mathrm{~s}$ to $2400 \mathrm{~s}$, calculated for a $0.9 \mathrm{~m}$ telescope. Each finding chart has the local standard stars clearly numbered and the host galaxy marked -SN. North is to the top, East to the left and the image scale is shown at the bottom of each image.

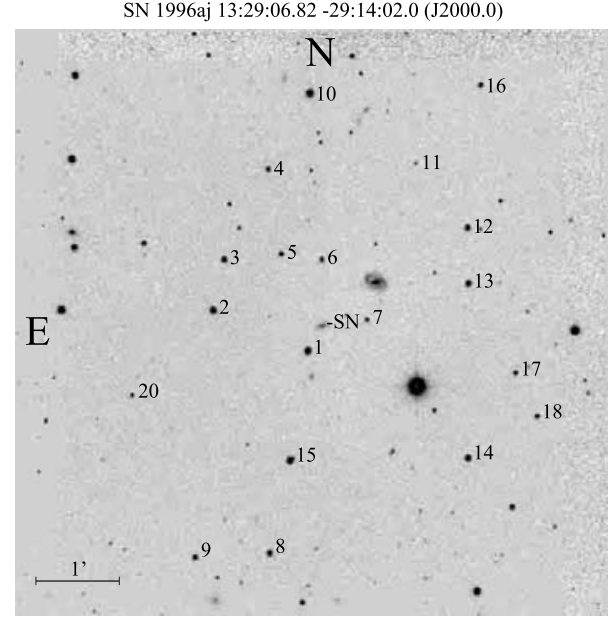

Fig. A.3. Finding chart for the local standard stars in the field of SN 1996aj.

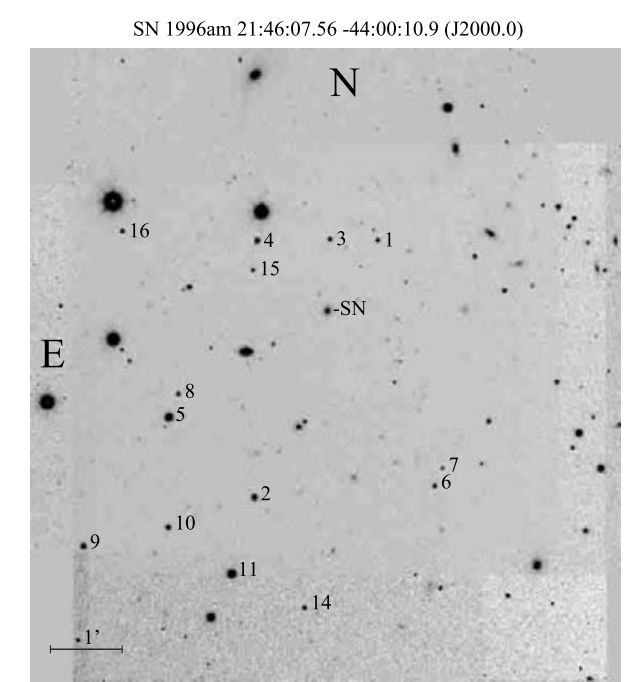

Fig. A.4. Finding chart for the local standard stars in the field of SN 1996am.

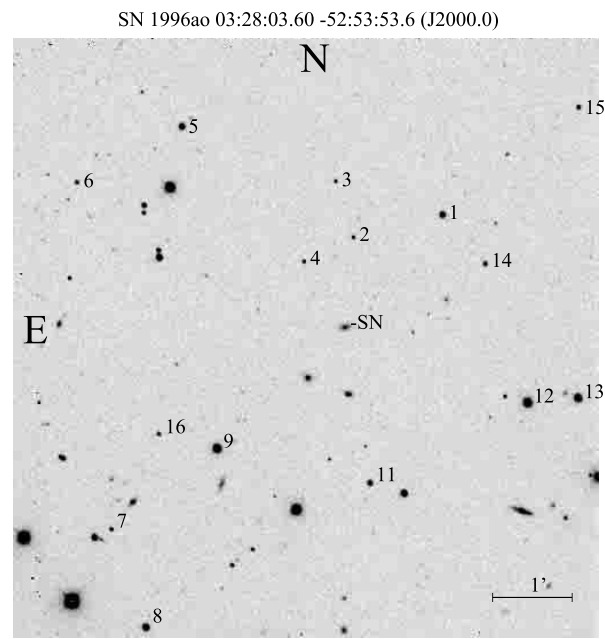

Fig. A.5. Finding chart for the local standard stars in the field of SN 1996ao. 
L. M. Germany et al.: Results Mount Stromlo Abell cluster SN search, Online Material $p 3$

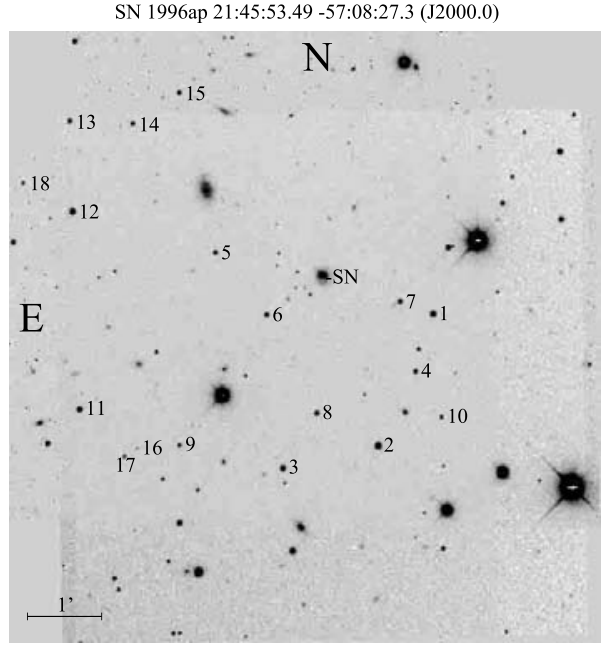

Fig. A.6. Finding chart for the local standard stars in the field of SN 1996ap.

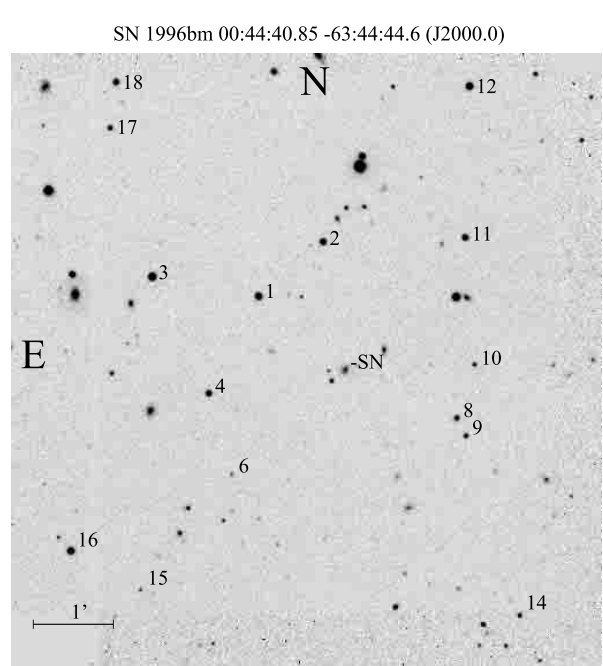

Fig. A.7. Finding chart for the local standard stars in the field of SN 1996bm.

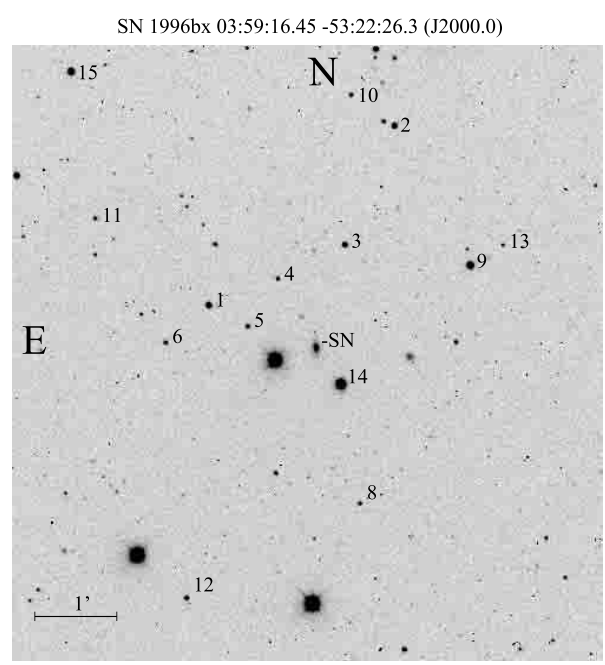

Fig. A.8. Finding chart for the local standard stars in the field of SN 1996bx.

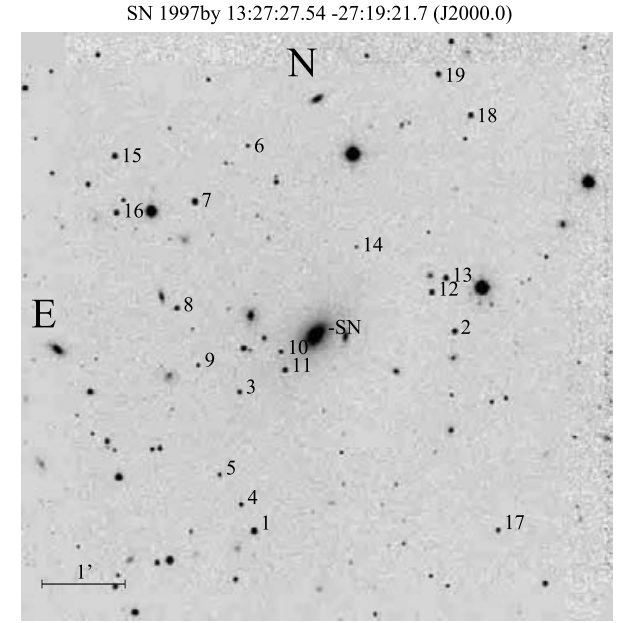

Fig. A.9. Finding chart for the local standard stars in the field of SN 1997by.

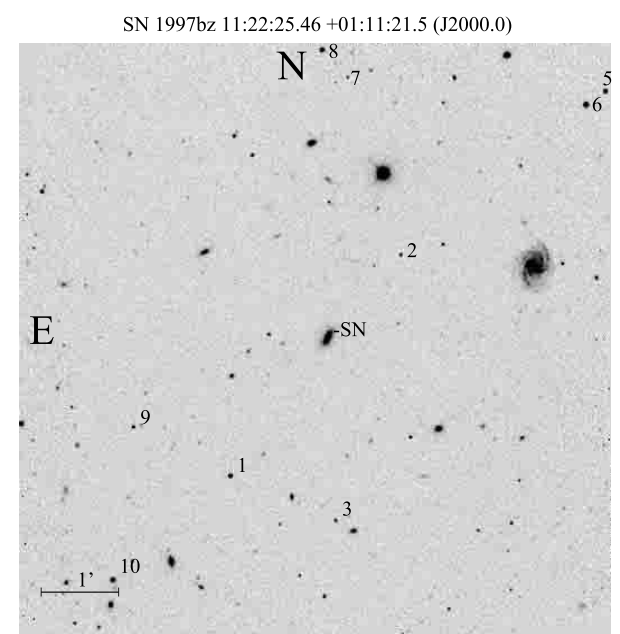

Fig. A.10. Finding chart for the local standard stars in the field of SN 1997bz.

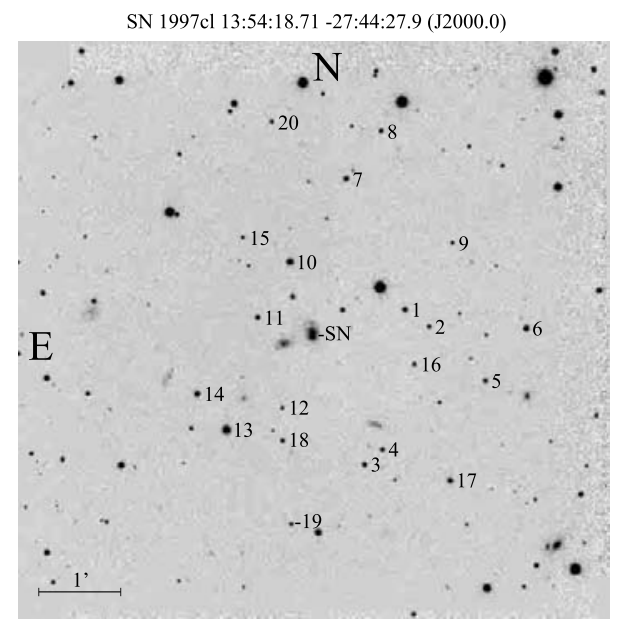

Fig. A.11. Finding chart for the local standard stars in the field of SN $1997 \mathrm{cl}$. 
L. M. Germany et al.: Results Mount Stromlo Abell cluster SN search, Online Material p 4

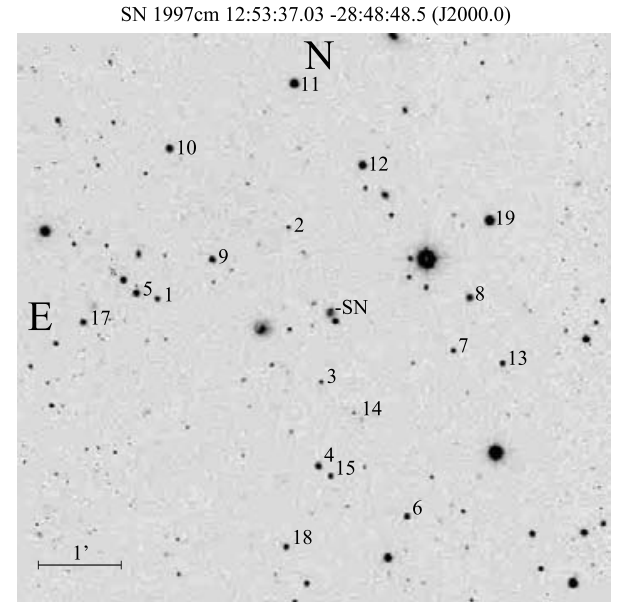

Fig. A.12. Finding chart for the local standard stars in the field of SN $1997 \mathrm{~cm}$.

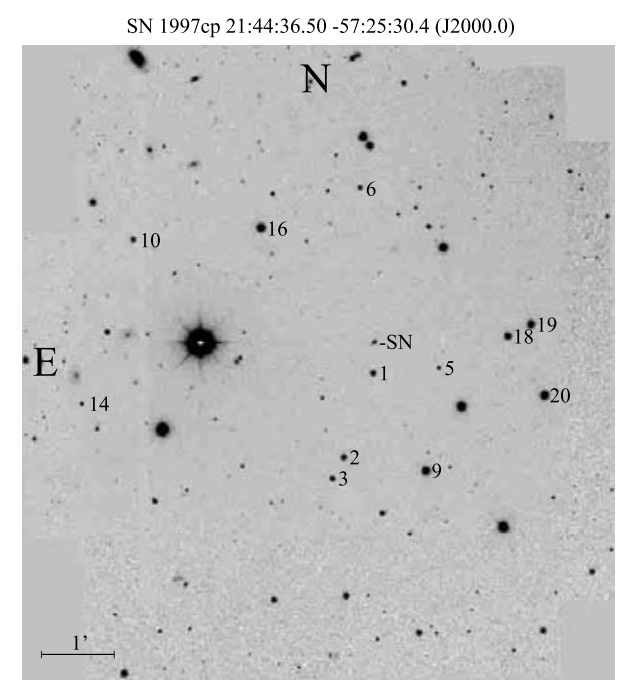

Fig. A.13. Finding chart for the local standard stars in the field of SN $1997 \mathrm{cp}$.

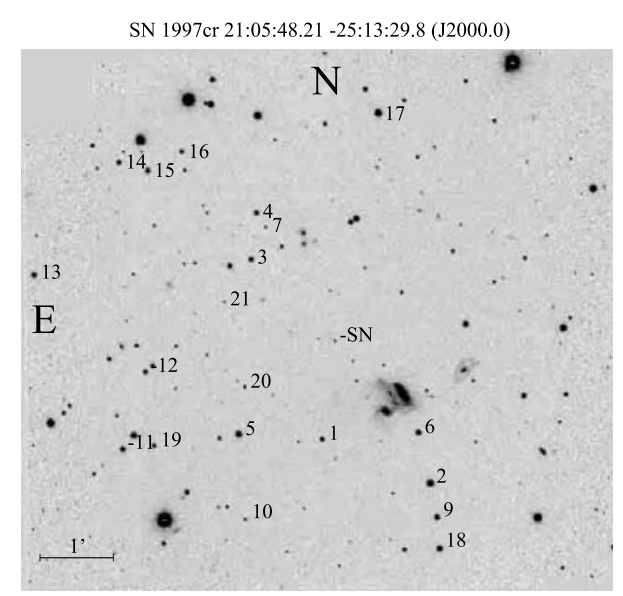

Fig. A.14. Finding chart for the local standard stars in the field of SN $1997 \mathrm{cr}$.

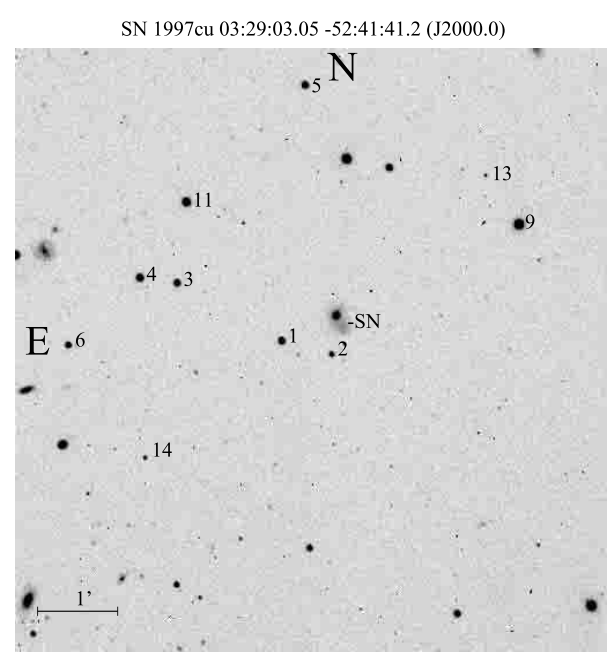

Fig. A.15. Finding chart for the local standard stars in the field of SN 1997cu.

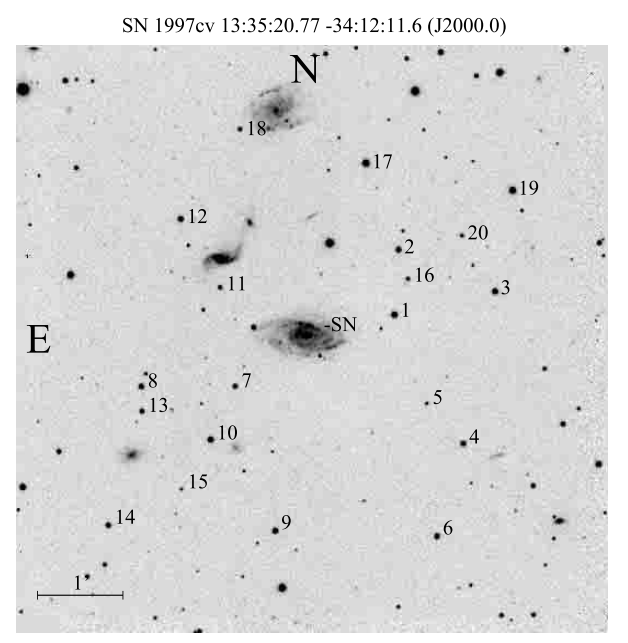

Fig. A.16. Finding chart for the local standard stars in the field of SN $1997 \mathrm{cv}$.

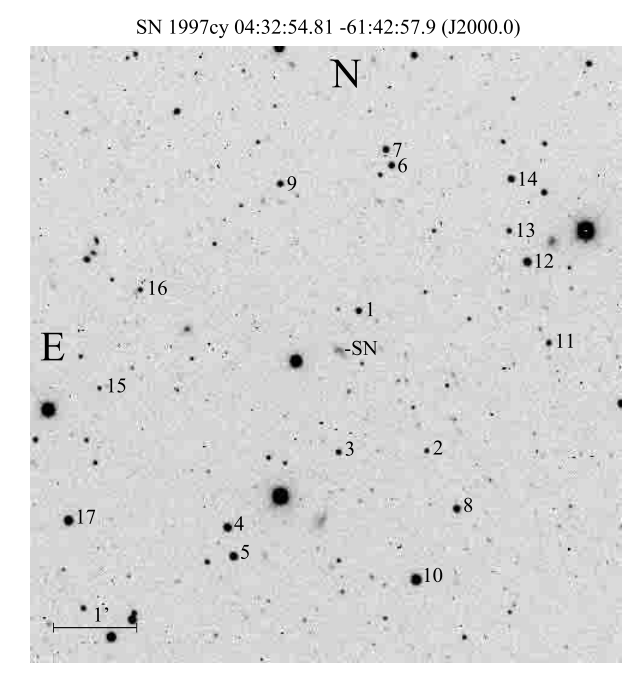

Fig. A.17. Finding chart for the local standard stars in the field of SN 1997cy. 
L. M. Germany et al.: Results Mount Stromlo Abell cluster SN search, Online Material p 5

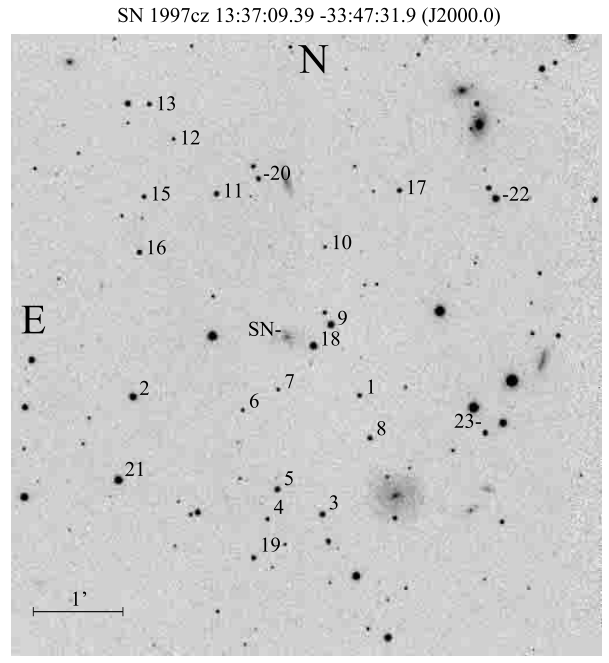

Fig. A.18. Finding chart for the local standard stars in the field of SN 1997cz.

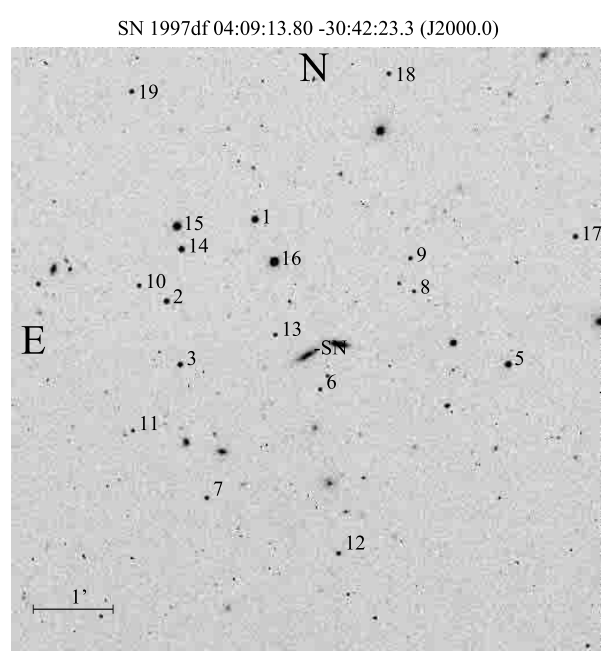

Fig. A.19. Finding chart for the local standard stars in the field of SN 1997 df.

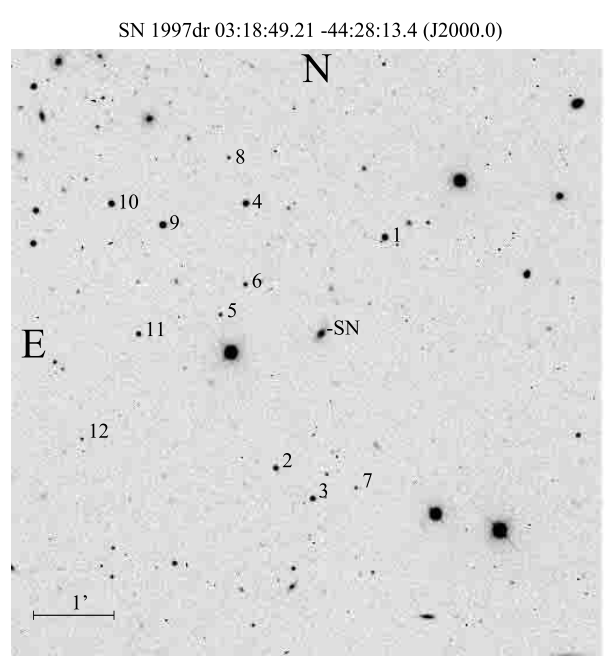

Fig. A.20. Finding chart for the local standard stars in the field of SN $1997 d r$.

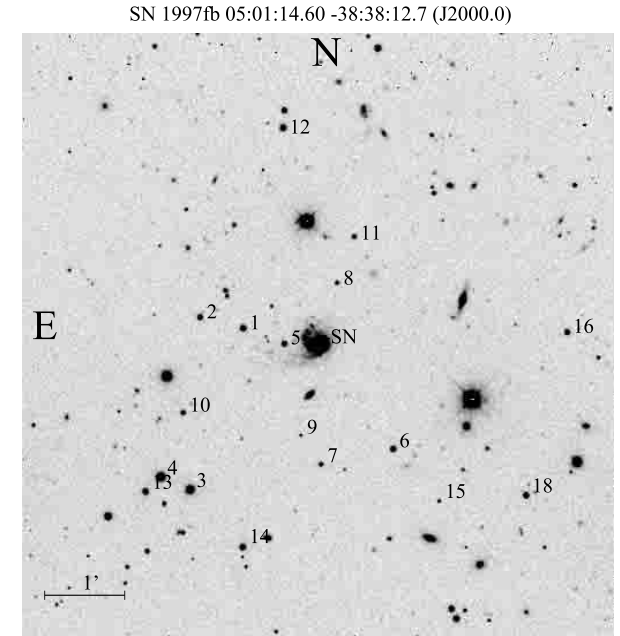

Fig. A.21. Finding chart for the local standard stars in the field of SN 1997fb.

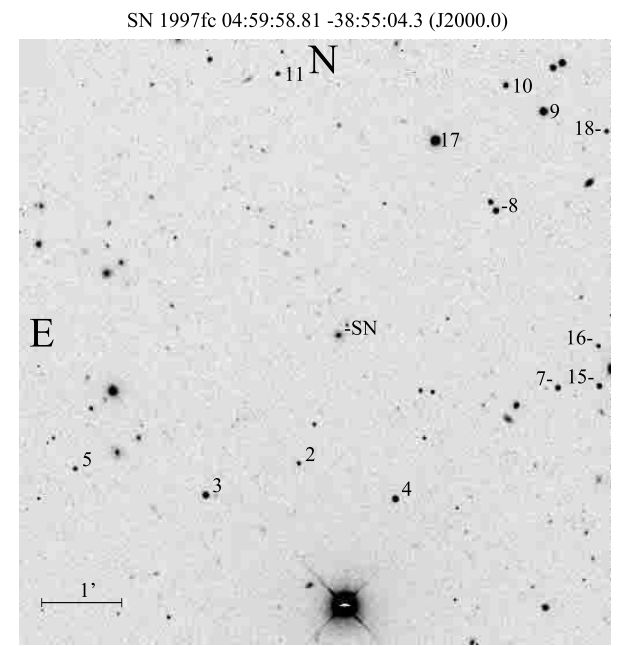

Fig. A.22. Finding chart for the local standard stars in the field of SN $1997 \mathrm{fc}$.

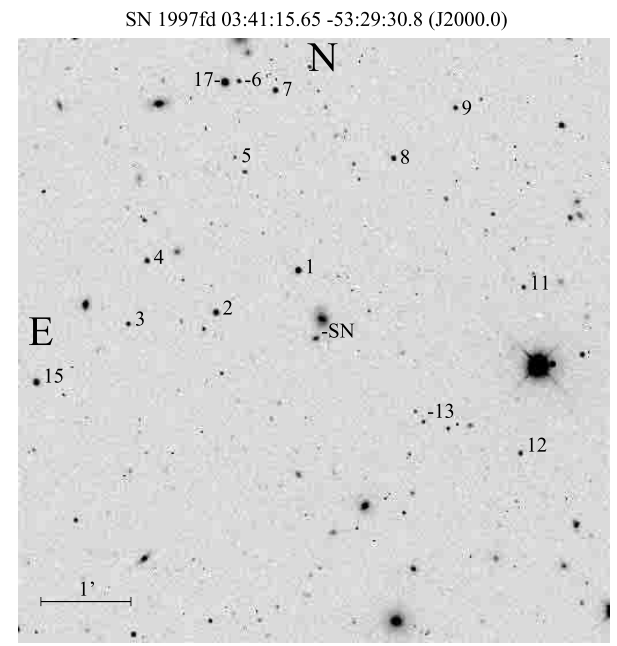

Fig. A.23. Finding chart for the local standard stars in the field of SN $1997 f$ f. 
L. M. Germany et al.: Results Mount Stromlo Abell cluster SN search, Online Material $p 6$

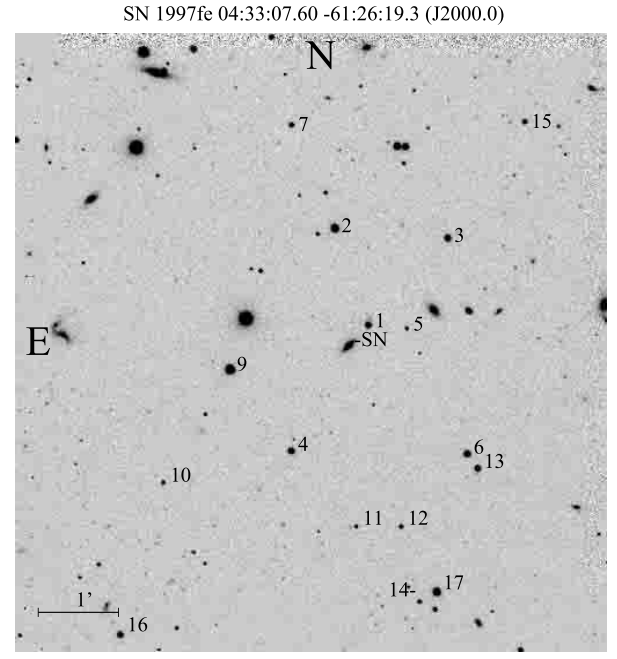

Fig. A.24. Finding chart for the local standard stars in the field of SN 1997fe.

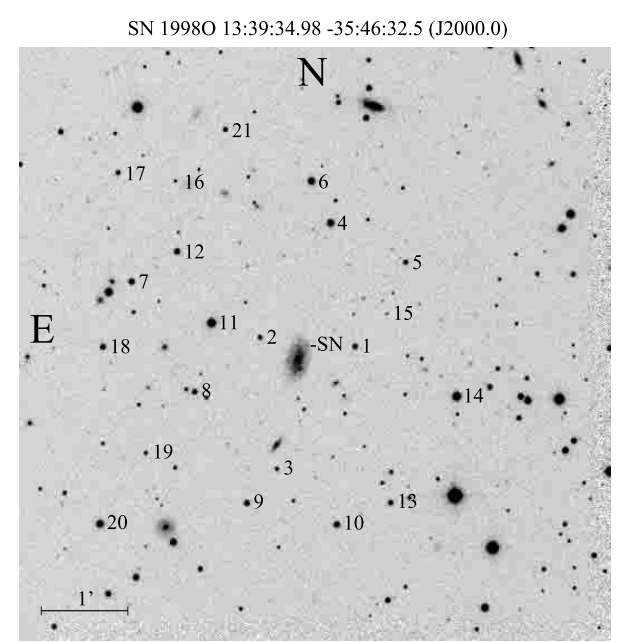

Fig. A.25. Finding chart for the local standard stars in the field of SN 19980.

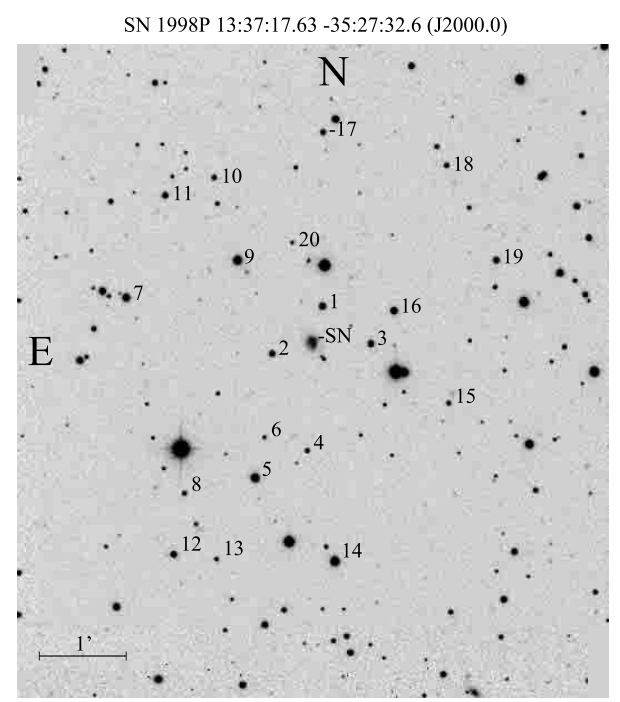

Fig. A.26. Finding chart for the local standard stars in the field of SN 1998P.

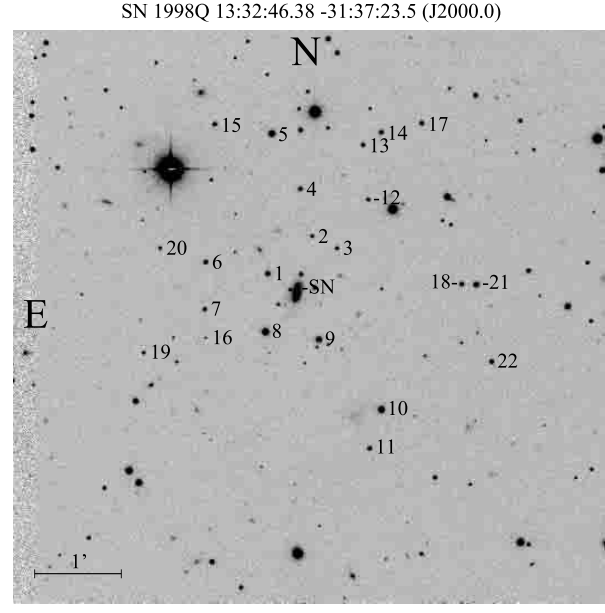

Fig. A.27. Finding chart for the local standard stars in the field of SN 1998Q.

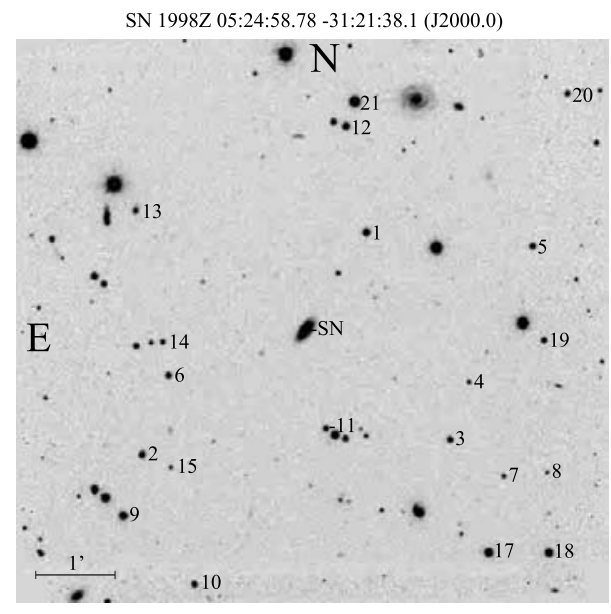

Fig. A.28. Finding chart for the local standard stars in the field of SN $1998 Z$.

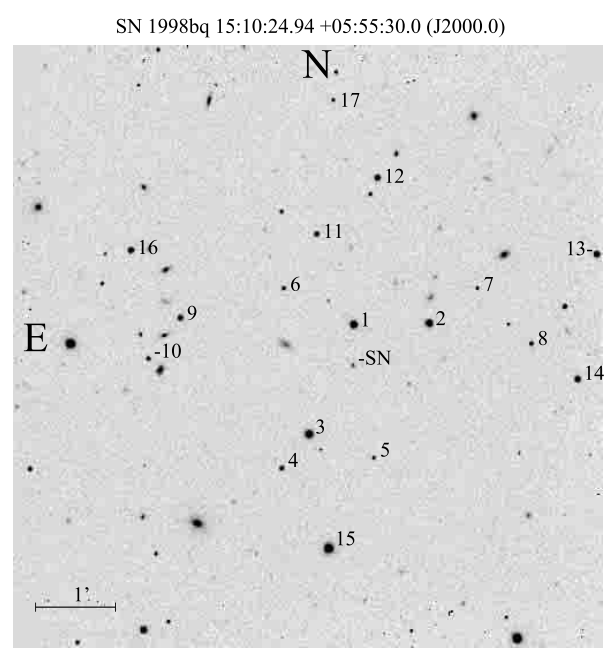

Fig. A.29. Finding chart for the local standard stars in the field of SN 1998bq. 
L. M. Germany et al.: Results Mount Stromlo Abell cluster SN search, Online Material $p 7$

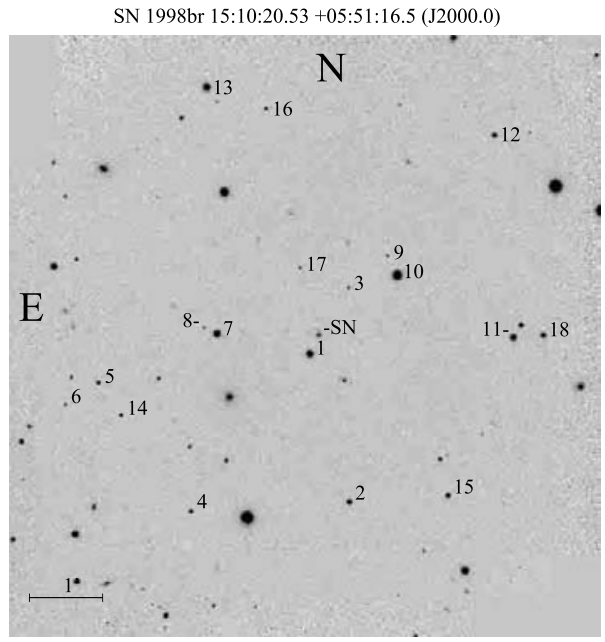

Fig. A.30. Finding chart for the local standard stars in the field of SN 1998br.

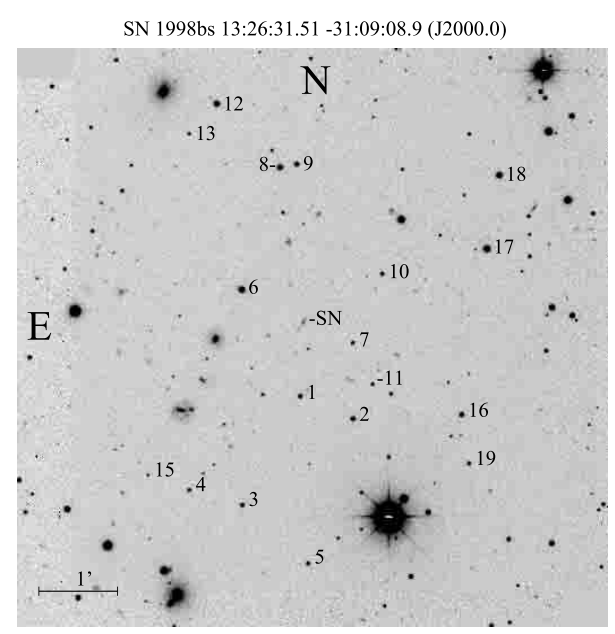

Fig. A.31. Finding chart for the local standard stars in the field of SN 1998bs.

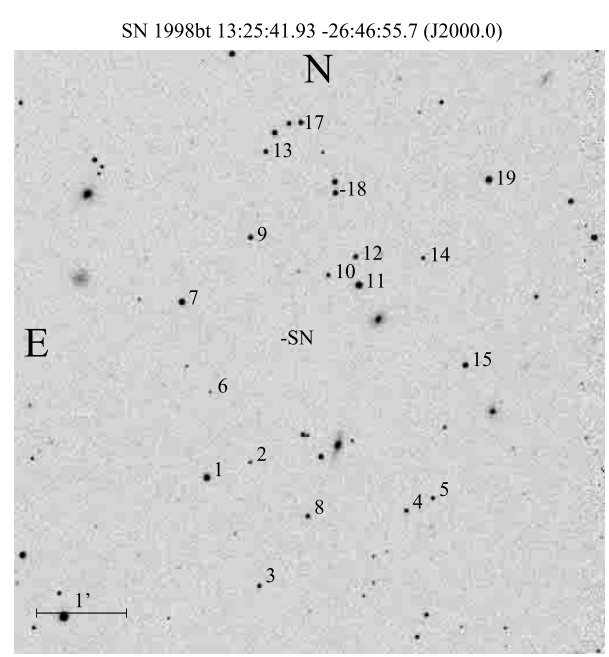

Fig. A.32. Finding chart for the local standard stars in the field of SN 1998bt.

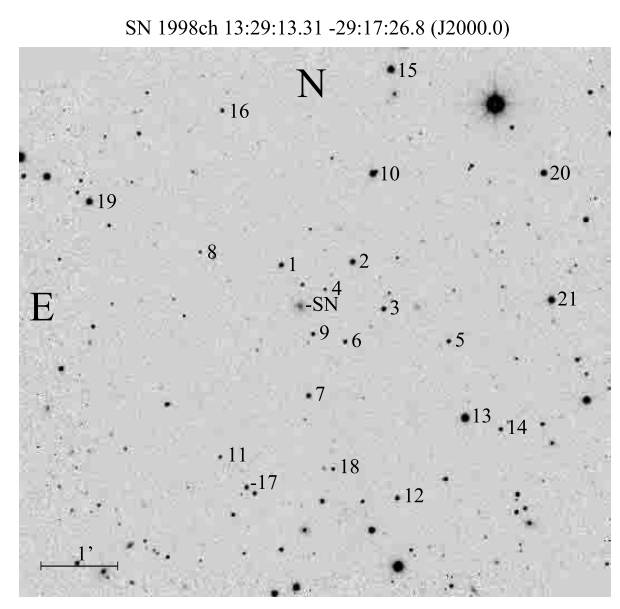

Fig. A.33. Finding chart for the local standard stars in the field of SN $1998 \mathrm{ch}$.

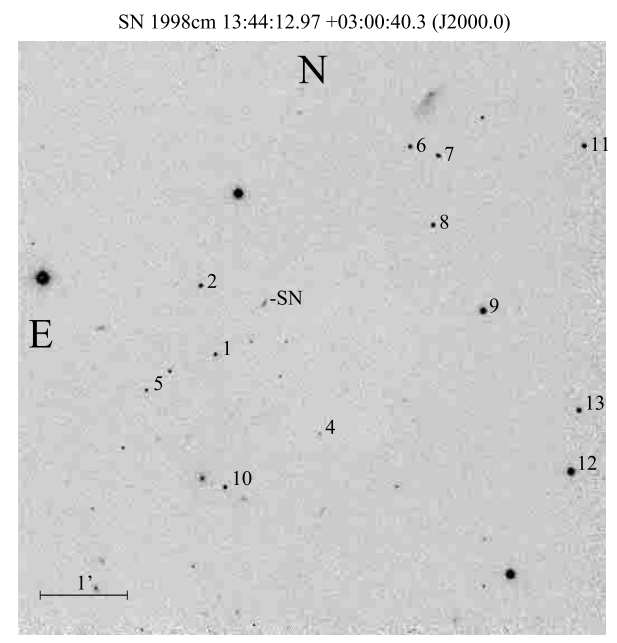

Fig. A.34. Finding chart for the local standard stars in the field of SN $1998 \mathrm{~cm}$.

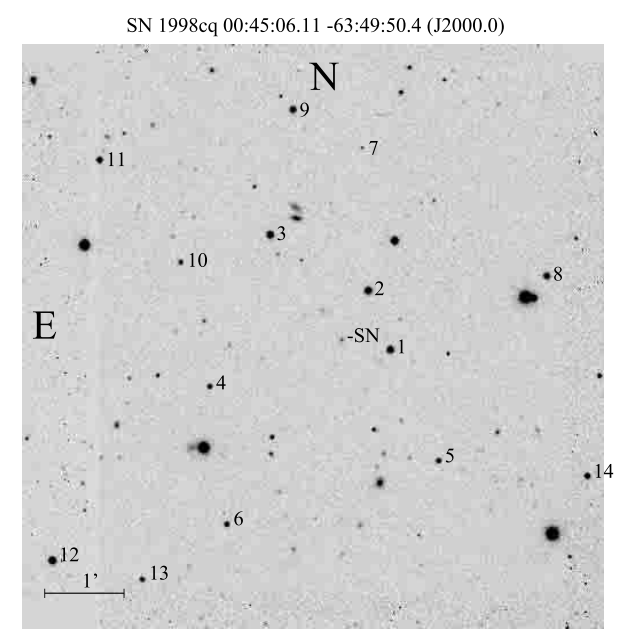

Fig. A.35. Finding chart for the local standard stars in the field of SN 1998cq. 
L. M. Germany et al.: Results Mount Stromlo Abell cluster SN search, Online Material $p 8$

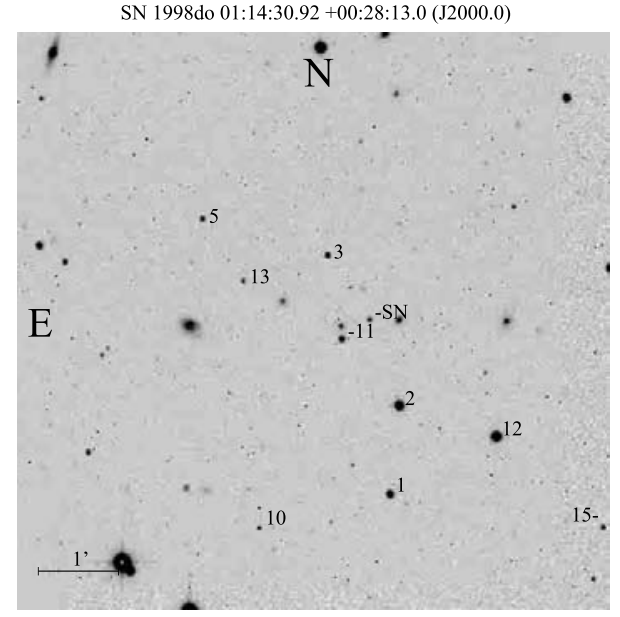

Fig. A.36. Finding chart for the local standard stars in the field of SN 1998do.

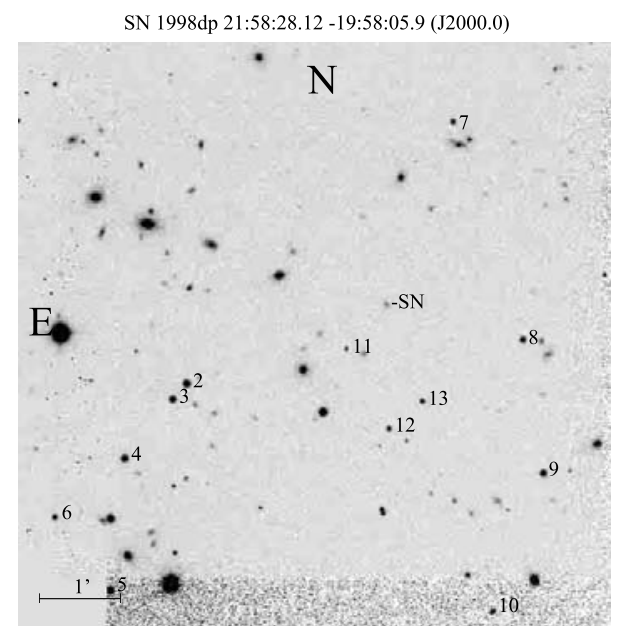

Fig. A.37. Finding chart for the local standard stars in the field of SN 1998dp.

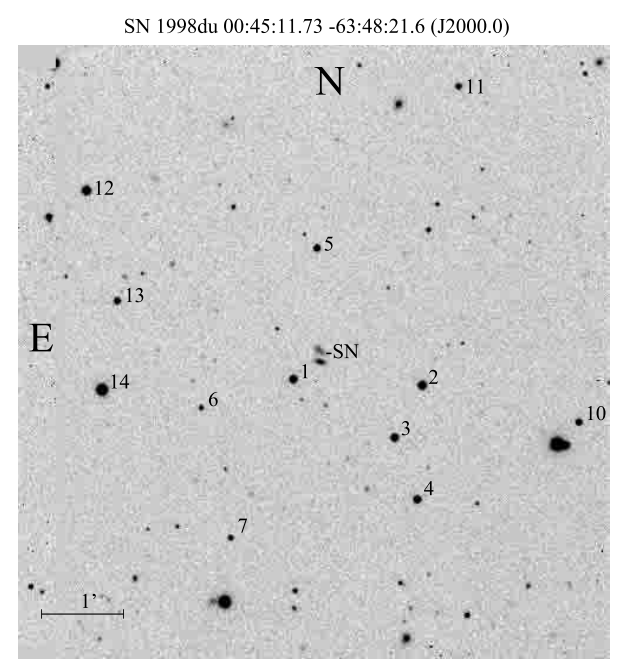

Fig. A.38. Finding chart for the local standard stars in the field of SN 1998 du.

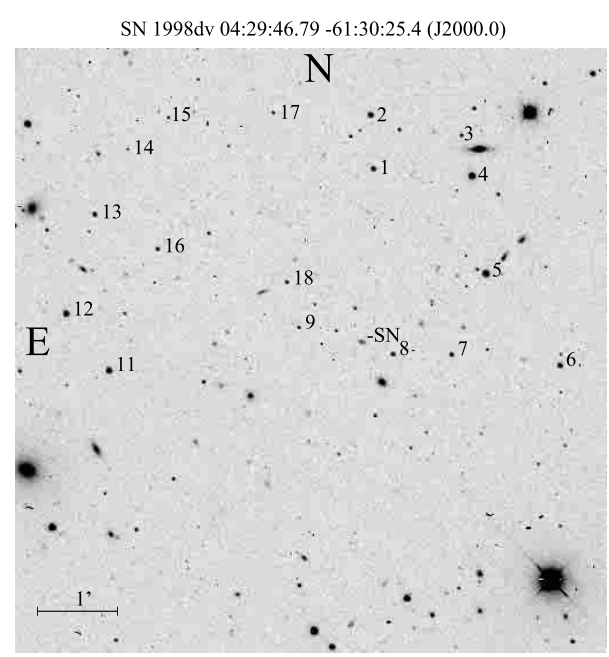

Fig. A.39. Finding chart for the local standard stars in the field of SN $1998 \mathrm{dv}$.

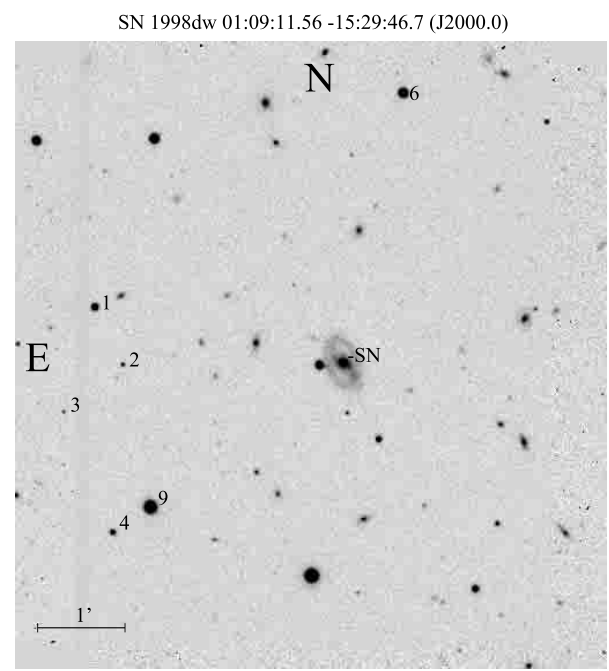

Fig. A.40. Finding chart for the local standard stars in the field of SN 1998dw.

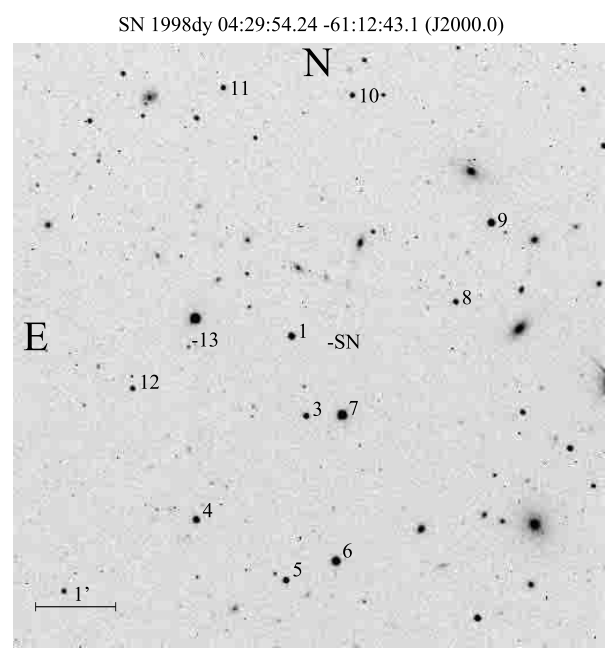

Fig. A.41. Finding chart for the local standard stars in the field of SN 1998dy. 
L. M. Germany et al.: Results Mount Stromlo Abell cluster SN search, Online Material $p 9$

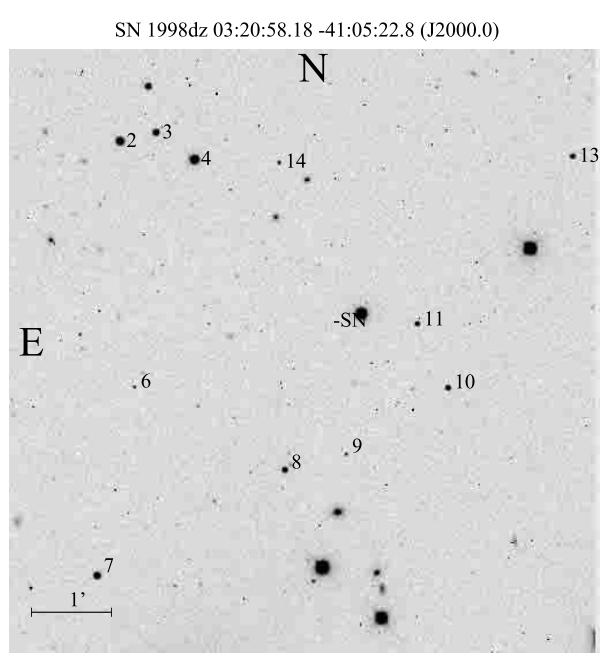

Fig. A.42. Finding chart for the local standard stars in the field of SN 1998dz.

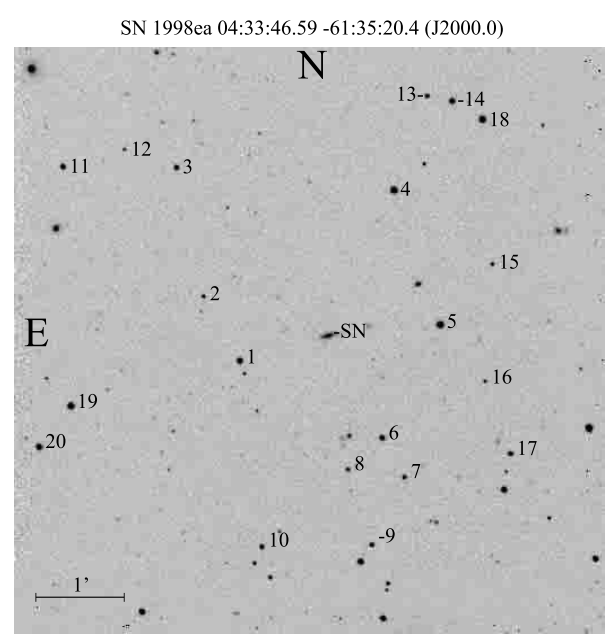

Fig. A.43. Finding chart for the local standard stars in the field of SN 1998ea.

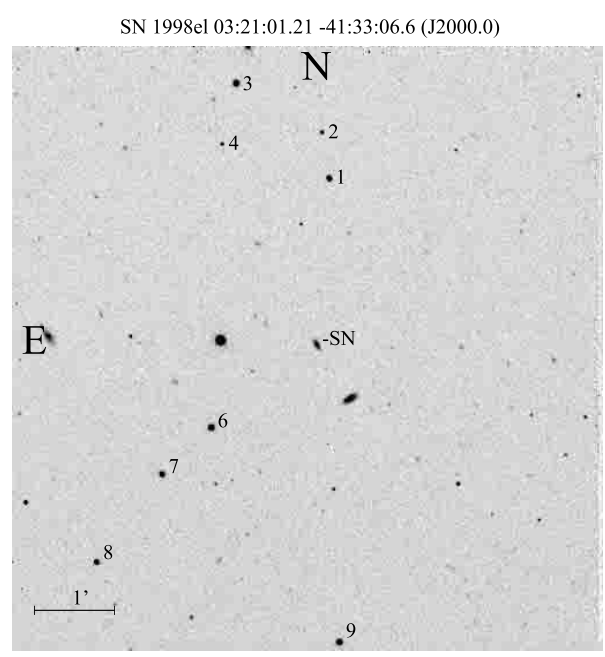

Fig. A.44. Finding chart for the local standard stars in the field of SN 1998el.

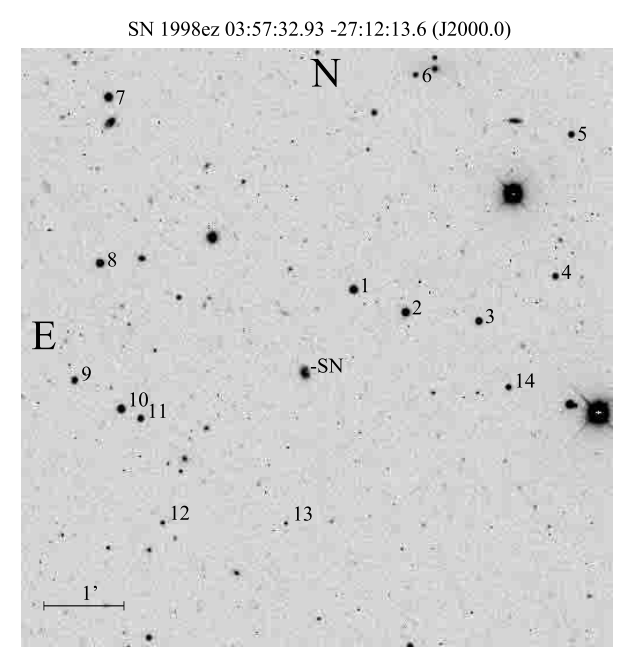

Fig. A.45. Finding chart for the local standard stars in the field of $\mathrm{SN}$ 1998ez.

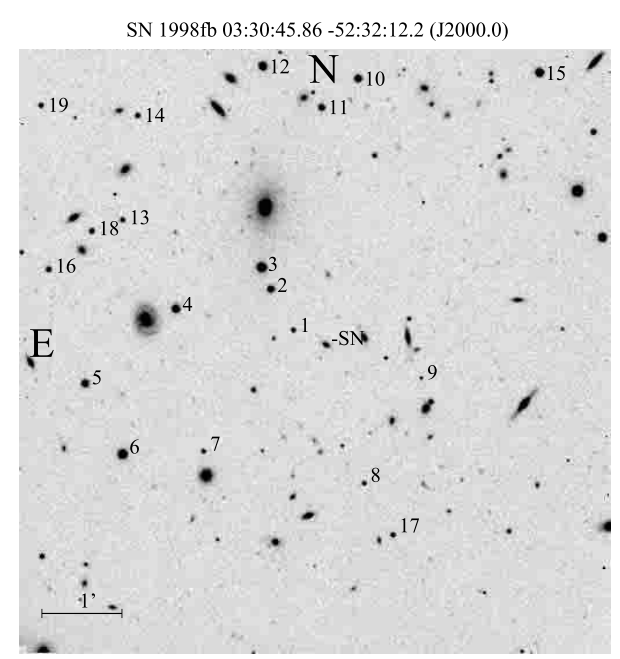

Fig. A.46. Finding chart for the local standard stars in the field of SN $1998 \mathrm{fb}$.

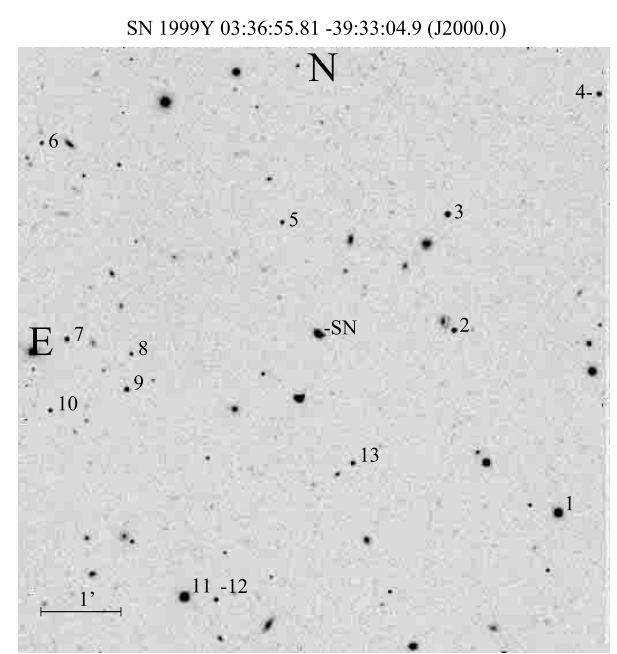

Fig. A.47. Finding chart for the local standard stars in the field of SN 1999Y. 
L. M. Germany et al.: Results Mount Stromlo Abell cluster SN search, Online Material p 10

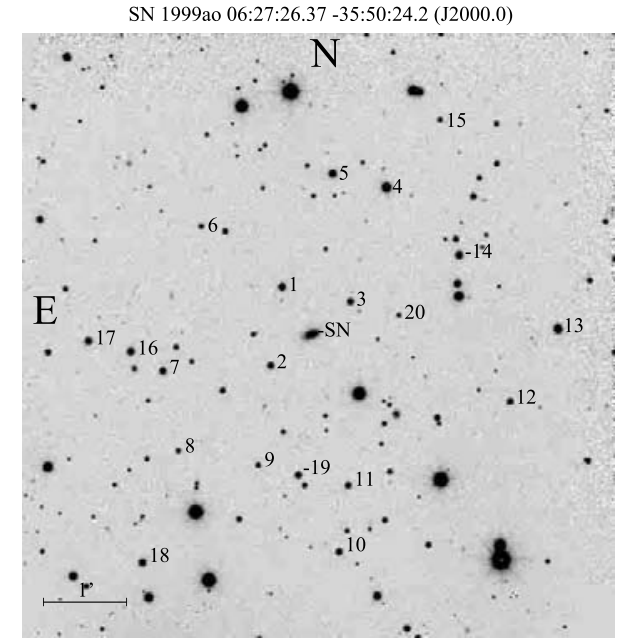

Fig. A.48. Finding chart for the local standard stars in the field of SN 1999ao.

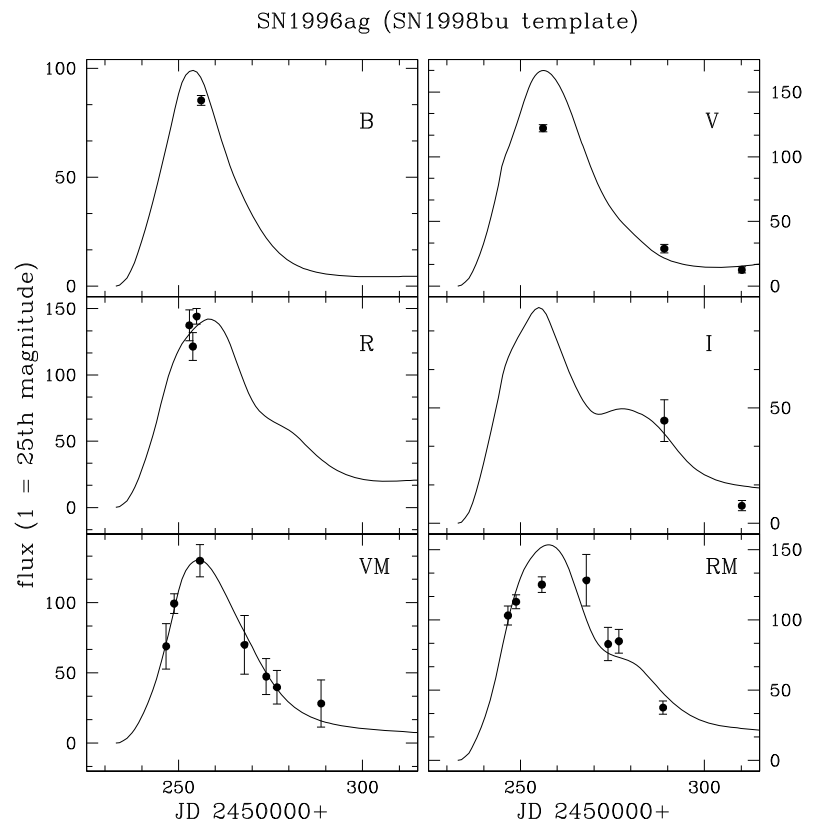

Fig. B.1. $B, V, R, I, V_{\mathrm{M}}$ and $R_{\mathrm{M}}$ light curves for $\mathrm{SN} 1996 \mathrm{ag}$. The solid line is the template that best fits the data-SN 1998bu.

\section{Appendix B: The SNla Light Curves}

In Sect. 4.4.2, template light curves were fit to the data for each $\mathrm{SN}$ via the $\Delta m_{15}$ method. In many cases, the goodness of the template fit to the data determined whether or not the SN was Type Ia. This Appendix shows the light curves and best-fit template (in units of flux) in each colour for those SNe discovered during the Mount Stromlo Abell cluster SN search that were deemed to be SNIa.

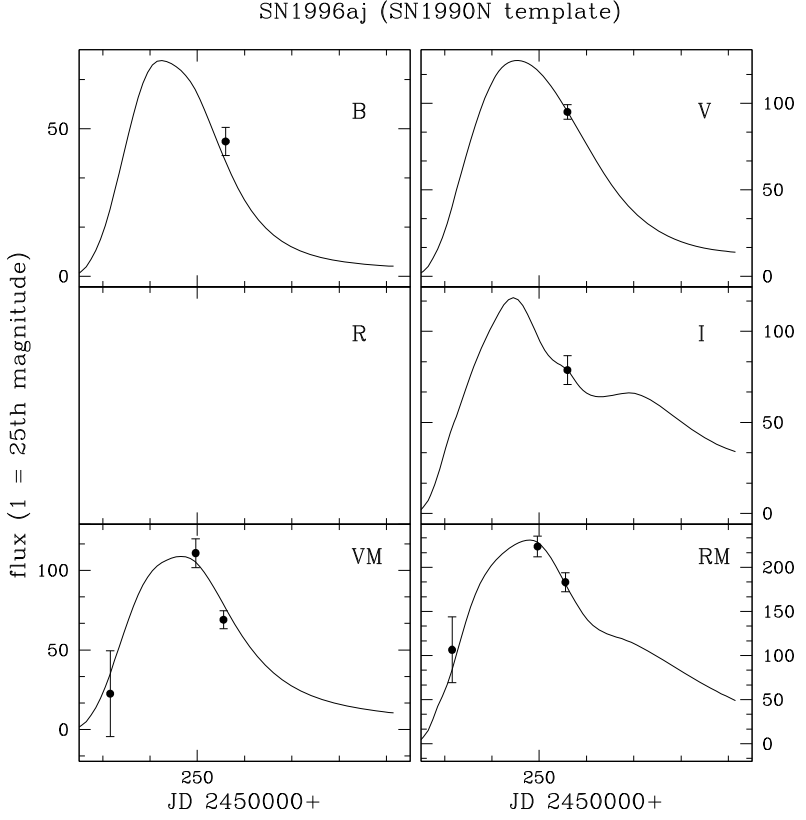

Fig. B.2. $B, V, I, V_{\mathrm{M}}$ and $R_{\mathrm{M}}$ light curves for $\mathrm{SN}$ 1996aj. The solid line is the template that best fits the data- SN $1990 \mathrm{~N}$.

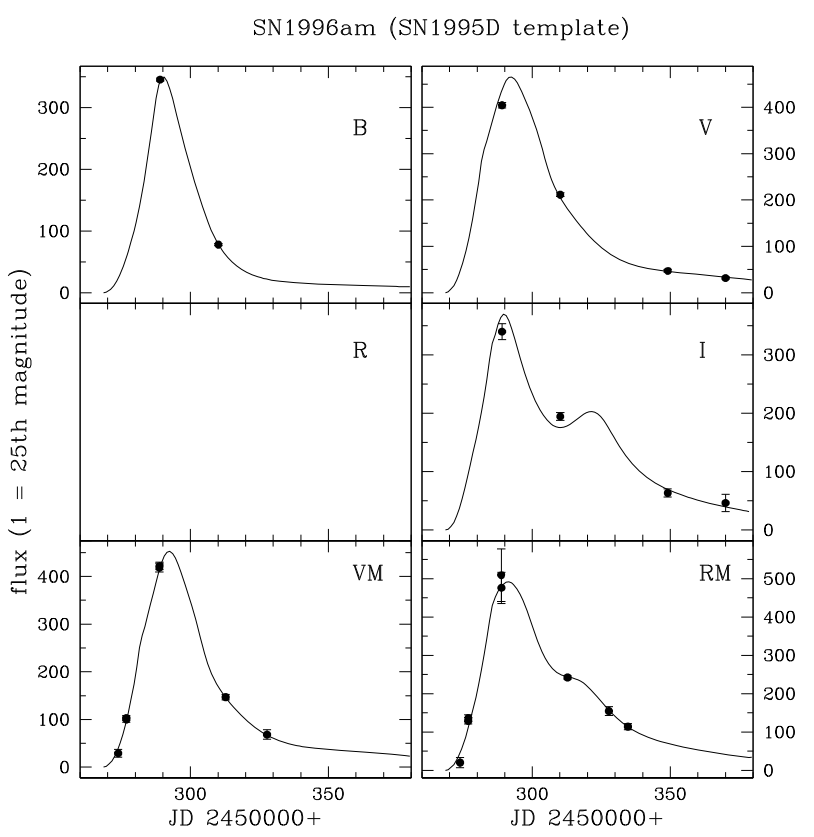

Fig. B.3. $B, V, I, V_{\mathrm{M}}$ and $R_{\mathrm{M}}$ light curves for $\mathrm{SN} 1996 \mathrm{am}$. The solid line is the template that best fits the data-SN 1995D. 
L. M. Germany et al.: Results Mount Stromlo Abell cluster SN search, Online Material p 11

SN1996ao (SN1994ae template)

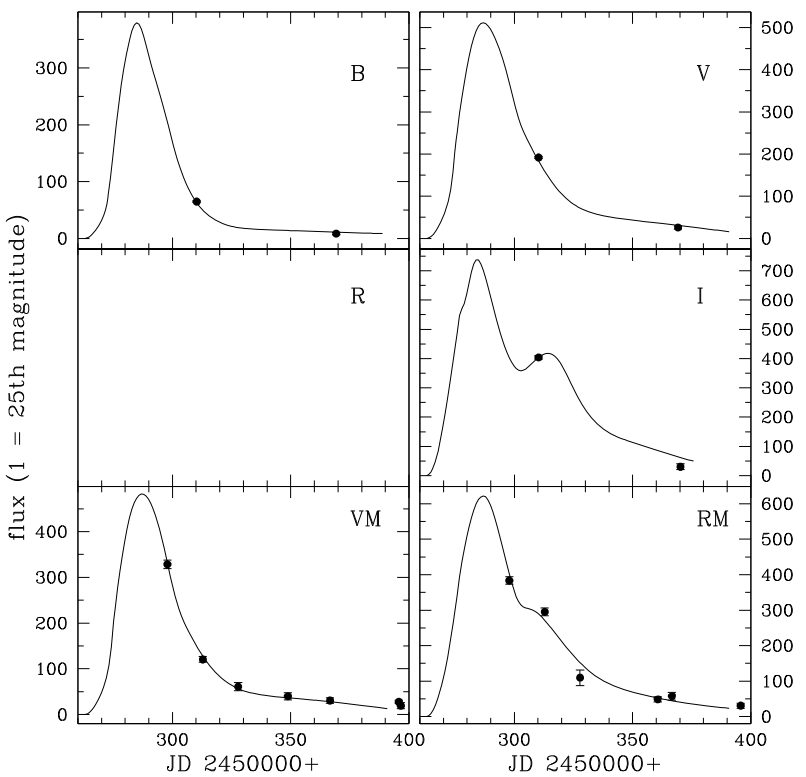

Fig. B.4. $B, V, I, V_{\mathrm{M}}$ and $R_{\mathrm{M}}$ light curves for $\mathrm{SN}$ 1996ao. The solid line is the template that best fits the data - SN 1994ae.

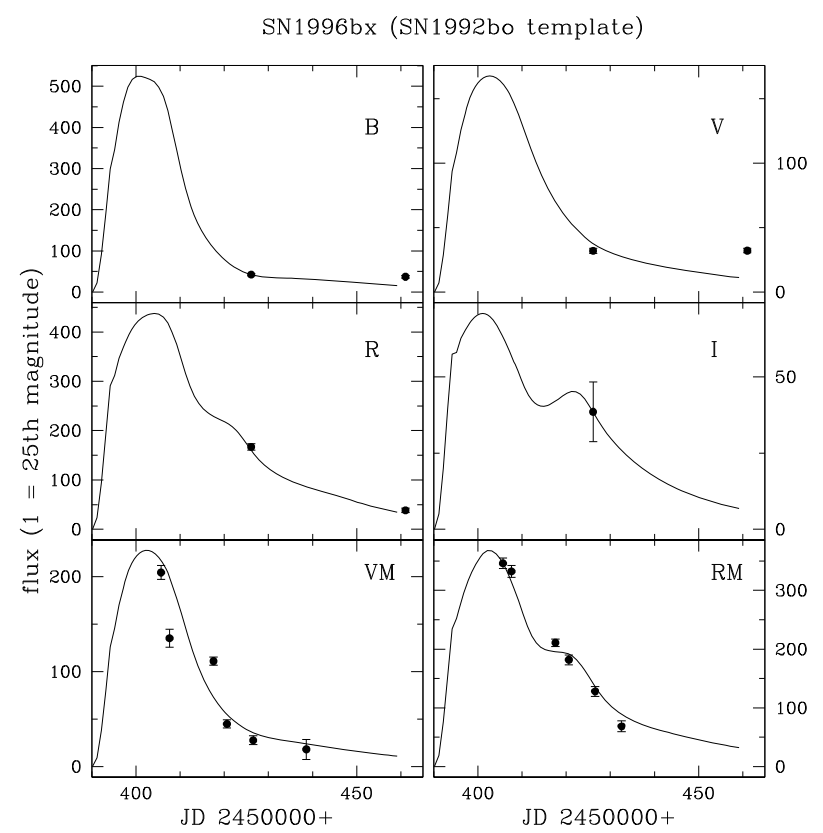

Fig. B.5. $B, V, R, I, V_{\mathrm{M}}$ and $R_{\mathrm{M}}$ light curves for $\mathrm{SN} 1996 \mathrm{bx}$. The solid line is the template that best fits the data-SN 1992 bo.
SN1997by (SN1992A template)

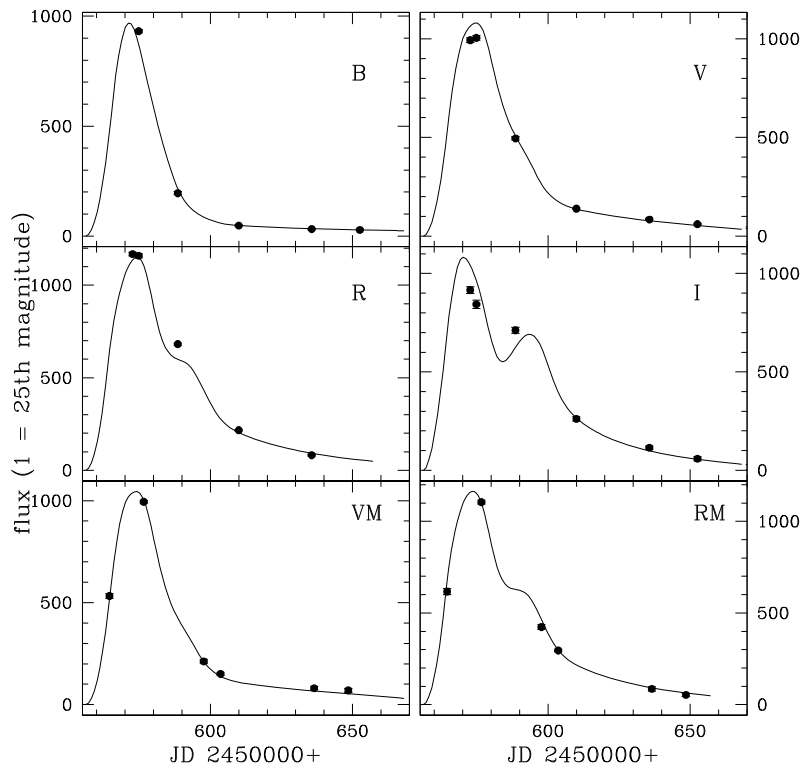

Fig. B.6. $B, V, R, I, V_{\mathrm{M}}$ and $R_{\mathrm{M}}$ light curves for $\mathrm{SN} 1997 \mathrm{by}$. The solid line is the template that best fits the data - SN 1992A.

SN1997bz (SN1995bd template)

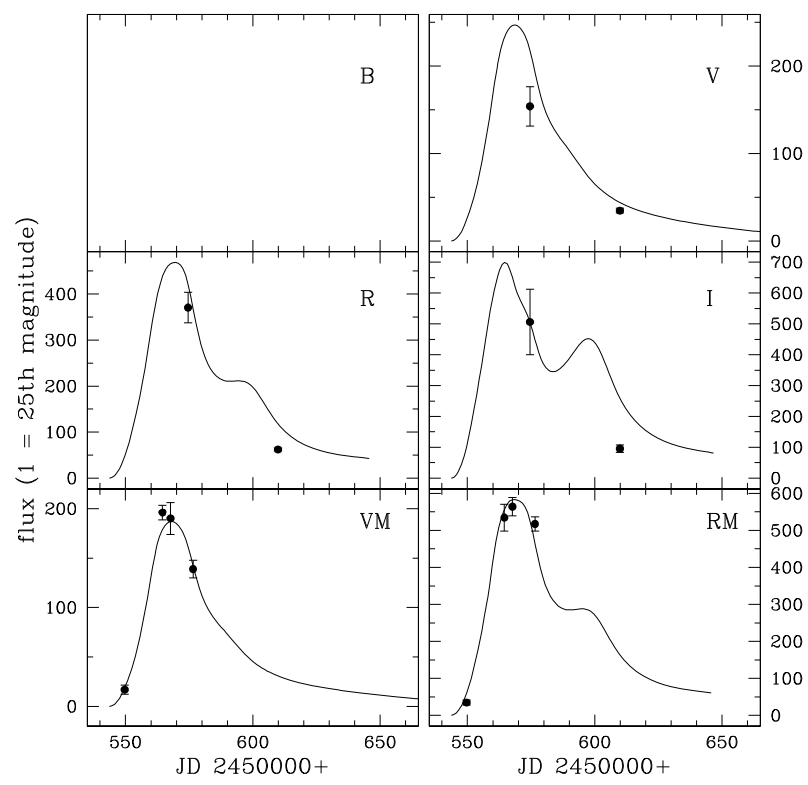

Fig. B.7. $V, R, I, V_{\mathrm{M}}$ and $R_{\mathrm{M}}$ light curves for $\mathrm{SN} 1997 \mathrm{bz}$. The solid line is the template that best fits the data-SN 1995bd.

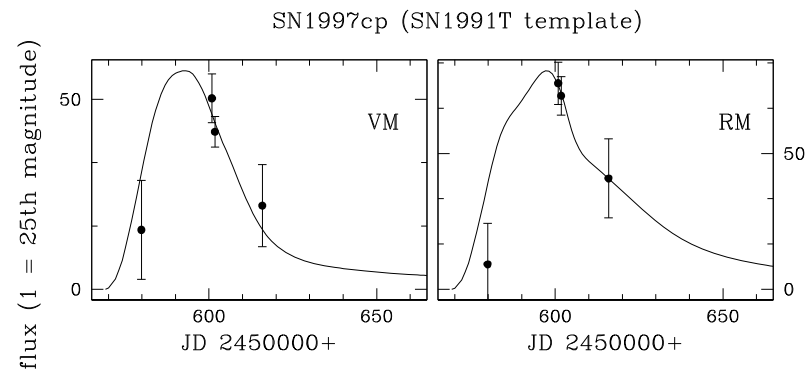

Fig. B.8. $V_{\mathrm{M}}$ and $R_{\mathrm{M}}$ light curves for $\mathrm{SN} 1997 \mathrm{cp}$. The solid line is the template that best fits the data - SN 1991T. 
L. M. Germany et al.: Results Mount Stromlo Abell cluster SN search, Online Material p 12

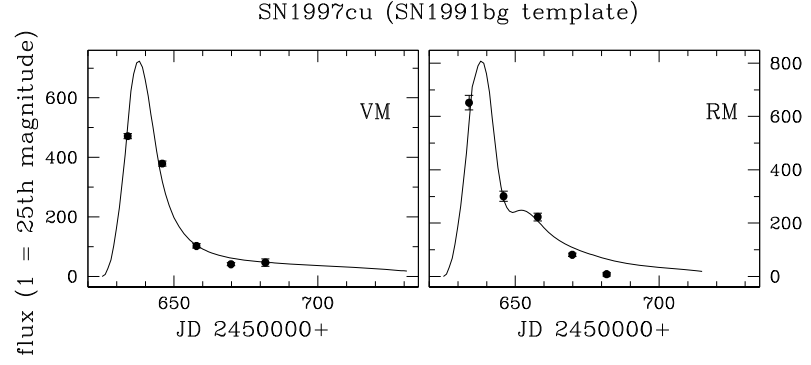

Fig. B.9. $V_{\mathrm{M}}$ and $R_{\mathrm{M}}$ light curves for $\mathrm{SN} 1997 \mathrm{cu}$. The solid line is the template that best fits the data - SN 1991 bg.

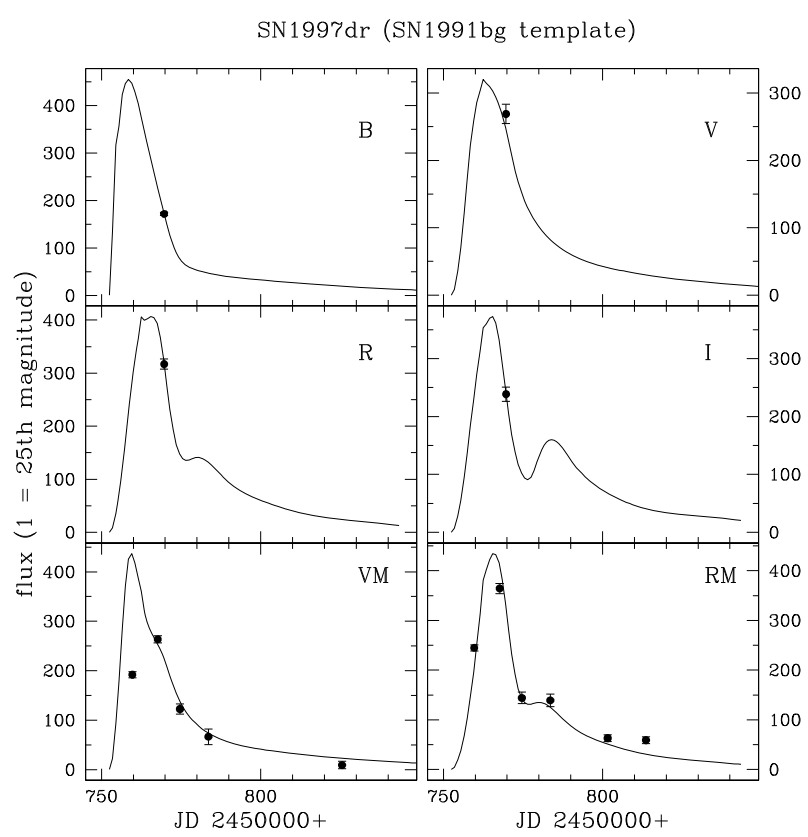

Fig. B.10. $B, V, R, I, V_{\mathrm{M}}$ and $R_{\mathrm{M}}$ light curves for $\mathrm{SN} 1997 \mathrm{dr}$. The solid line is the template that best fits the data-SN $1991 \mathrm{bg}$.

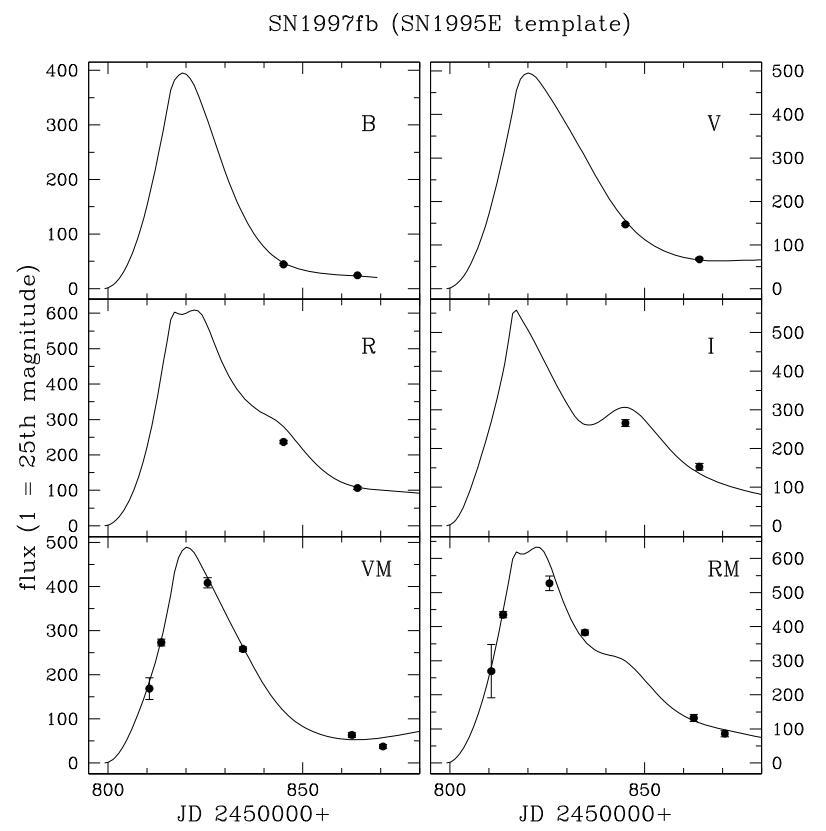

Fig. B.11. $B, V, R, I, V_{\mathrm{M}}$ and $R_{\mathrm{M}}$ light curves for $\mathrm{SN} 1997 \mathrm{fb}$. The solid line is the template that best fits the data- SN 1995E.

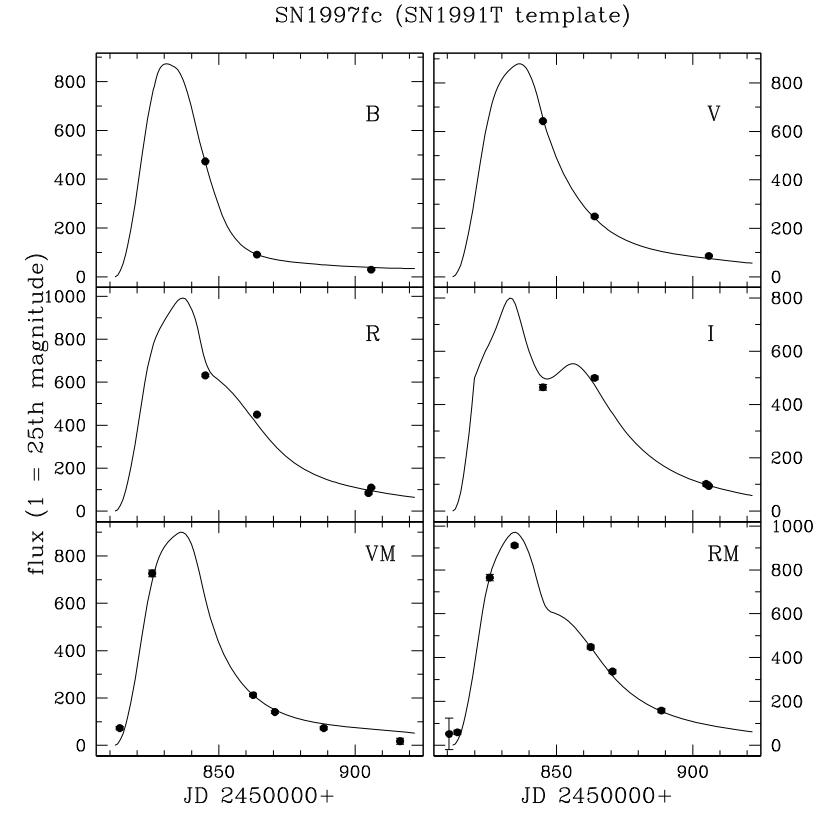

Fig. B.12. $B, V, R, I, V_{\mathrm{M}}$ and $R_{\mathrm{M}}$ light curves for $\mathrm{SN} 1997 \mathrm{fc}$. The solid line is the template that best fits the data-SN 1991T.

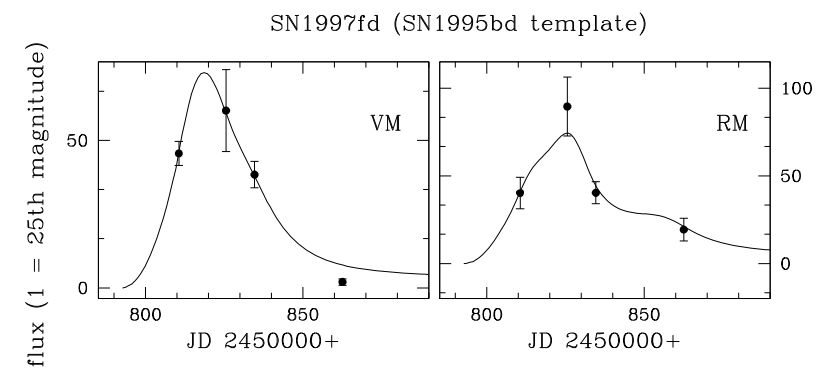

Fig. B.13. $V_{\mathrm{M}}$ and $R_{\mathrm{M}}$ light curves for $\mathrm{SN} 1997 \mathrm{fd}$. The solid line is the template that best fits the data - SN 1995bd.

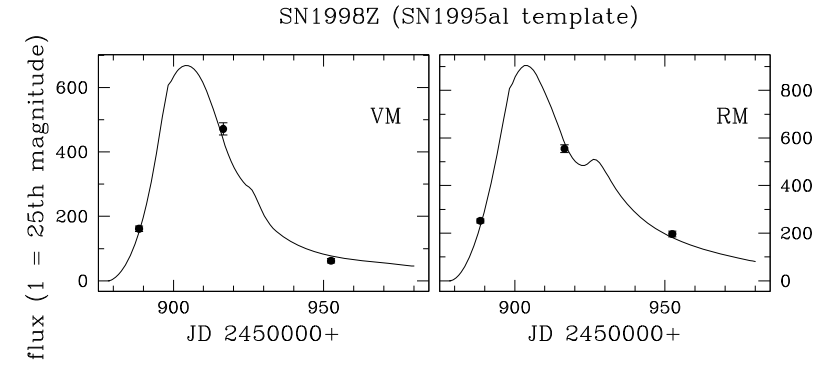

Fig. B.14. $V_{\mathrm{M}}$ and $R_{\mathrm{M}}$ light curves for $\mathrm{SN} 1998 \mathrm{Z}$. The solid line is the template that best fits the data - SN 1995al. 
L. M. Germany et al.: Results Mount Stromlo Abell cluster SN search, Online Material p 13

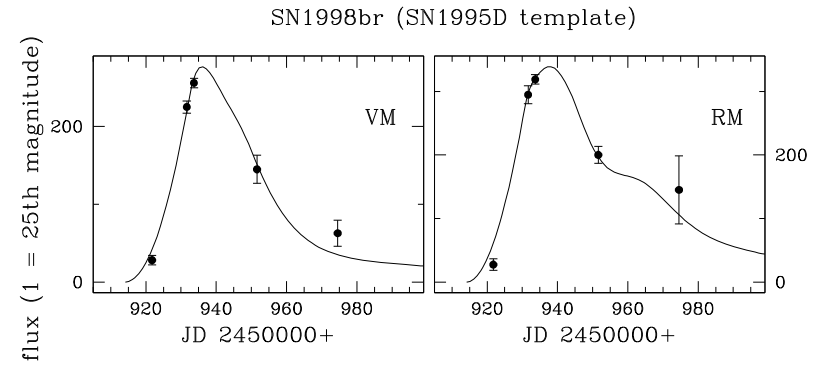

Fig. B.15. $V_{\mathrm{M}}$ and $R_{\mathrm{M}}$ light curves for $\mathrm{SN} 1998 \mathrm{br}$. The solid line is the template that best fits the data-SN 1995D.

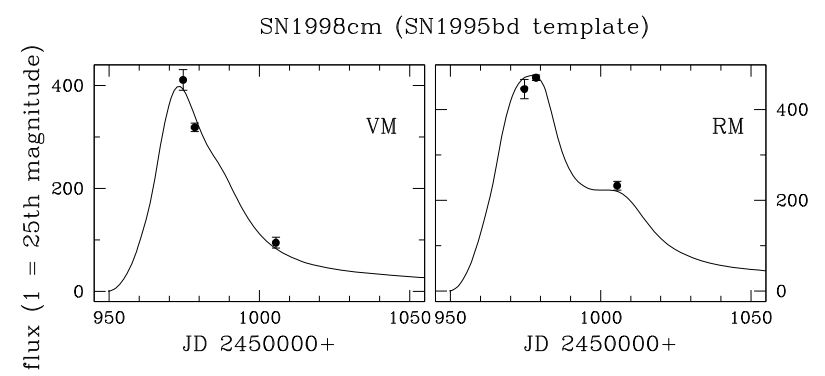

Fig. B.16. $V_{\mathrm{M}}$ and $R_{\mathrm{M}}$ light curves for $\mathrm{SN} 1998 \mathrm{~cm}$. The solid line is the template that best fits the data - SN 1995bd.

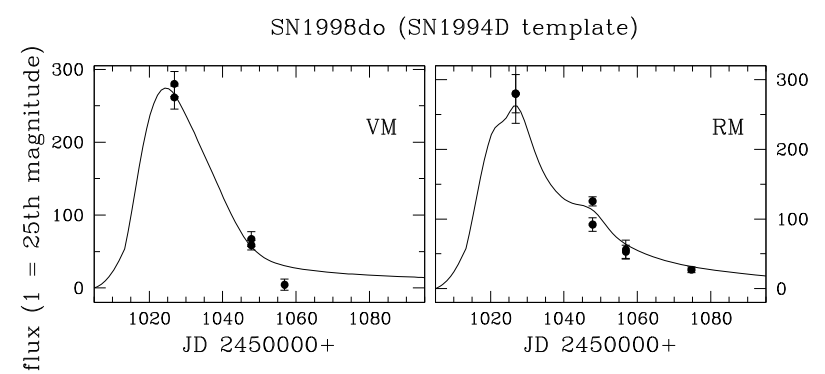

Fig. B.17. $V_{\mathrm{M}}$ and $R_{\mathrm{M}}$ light curves for $\mathrm{SN} 1998$ do. The solid line is the template that best fits the data-SN 1994D.

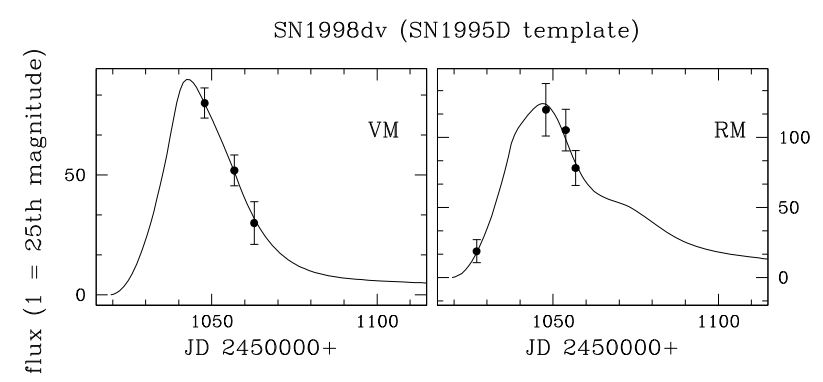

Fig. B.18. $V_{\mathrm{M}}$ and $R_{\mathrm{M}}$ light curves for $\mathrm{SN} 1998 \mathrm{dv}$. The solid line is the template that best fits the data-SN 1995D.

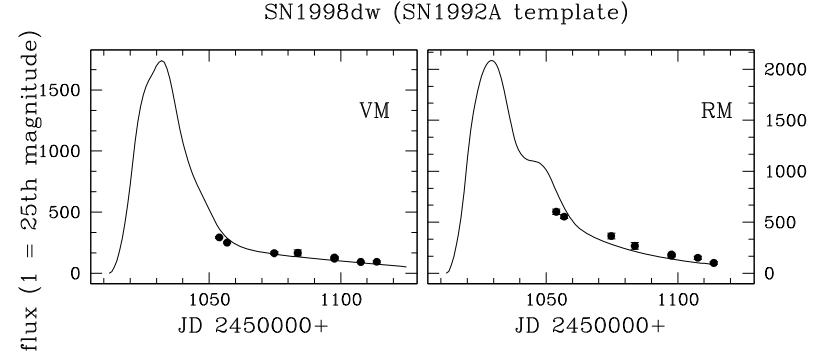

Fig. B.19. $V_{\mathrm{M}}$ and $R_{\mathrm{M}}$ light curves for $\mathrm{SN} 1998 \mathrm{dw}$. The solid line is the template that best fits the data - SN 1992A.

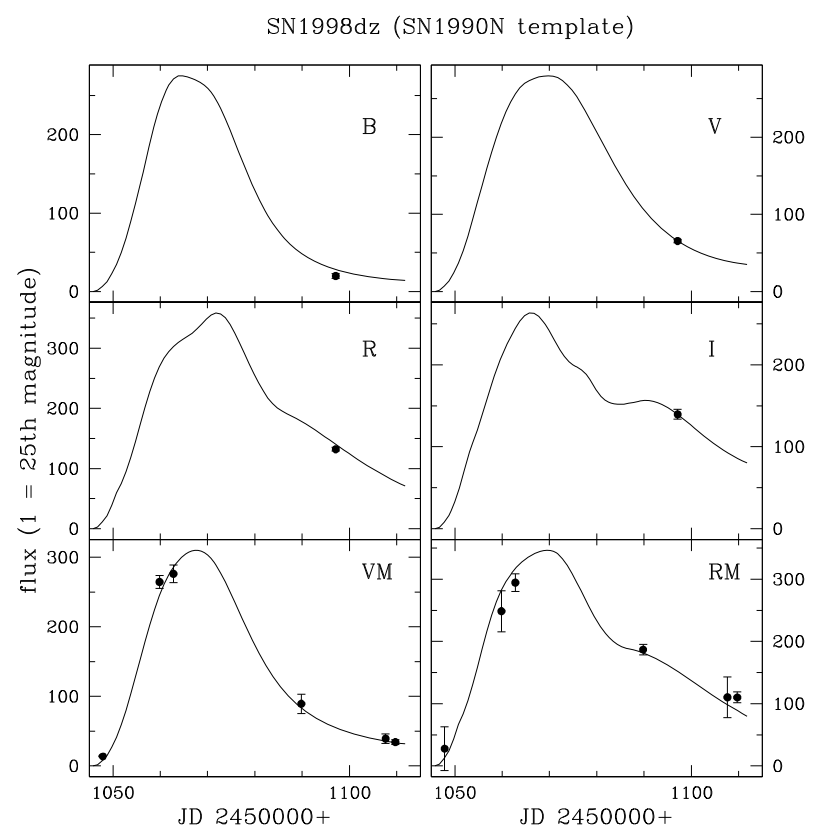

Fig. B.20. $B, V, R, I, V_{\mathrm{M}}$ and $R_{\mathrm{M}}$ light curves for $\mathrm{SN} 1998 \mathrm{dz}$. The solid line is the template that best fits the data - SN $1990 \mathrm{~N}$.

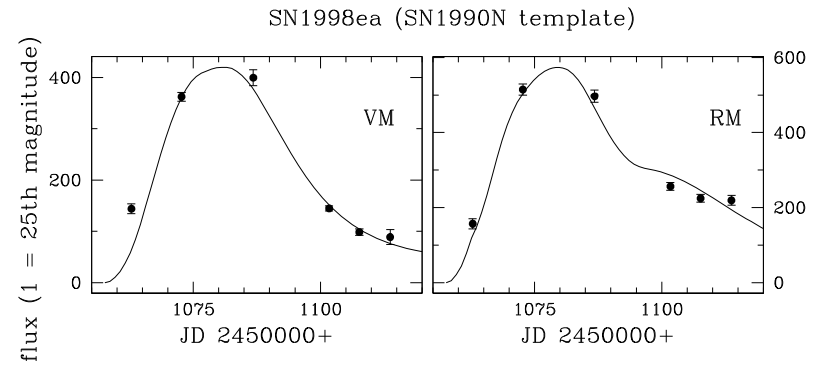

Fig. B.21. $V_{\mathrm{M}}$ and $R_{\mathrm{M}}$ light curves for $\mathrm{SN} 1998 \mathrm{ea}$. The solid line is the template that best fits the data-SN 1990 N. 
L. M. Germany et al.: Results Mount Stromlo Abell cluster SN search, Online Material p 14 SN1998fb (SN1992bo template)

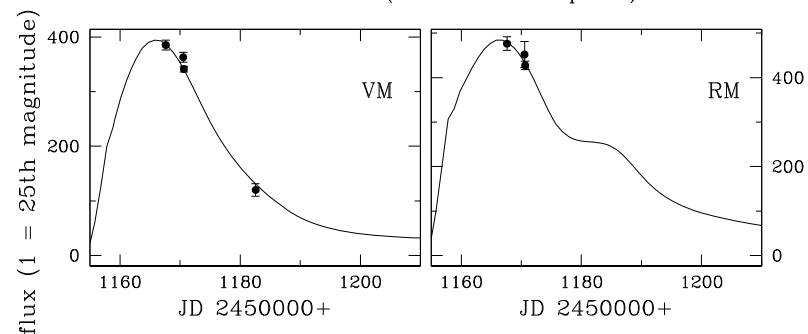

Fig. B.22. $V_{\mathrm{M}}$ and $R_{\mathrm{M}}$ light curves for $\mathrm{SN} 1998 \mathrm{fb}$. The solid line is the template that best fits the data $-\mathrm{SN} 1992 \mathrm{bo}$.

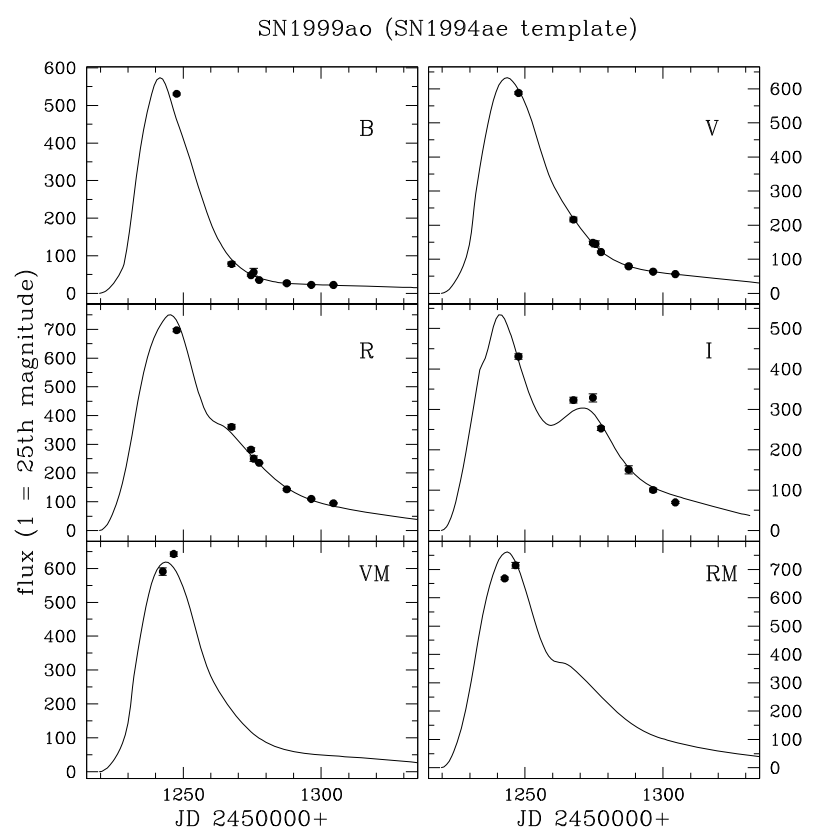

Fig. B.23. $B, V, R, I, V_{\mathrm{M}}$ and $R_{\mathrm{M}}$ light curves for $\mathrm{SN} 1999$ ao. The solid line is the template that best fits the data - SN 1994ae. 